\title{
15. CHEMISTRY AND GEOTHERMOMETRY OF SECONDARY MINERALS FROM THE DEEP SHEETED DIKE COMPLEX, HOLE 504B ${ }^{1}$
}

\author{
Christine Laverne, ${ }^{2}$ David A. Vanko, ${ }^{3}$ Paola Tartarotti, ${ }^{4}$ and Jeffrey C. $\mathrm{Alt}{ }^{5}$
}

\begin{abstract}
Dolerites sampled from the lower sheeted dikes from Hole 504B during Ocean Drilling Program Legs 137 and 140, between 1562.4 and $2000.4 \mathrm{mbsf}$, were examined to document the mineralogy, petrography, and mineral parageneses associated with secondary alteration, to constrain the thermal history and composition of hydrothermal fluids. The main methods used were mineral chemical analyses by electron microprobe, X-ray diffraction, and cathodoluminescence microscopy. Temperatures of alteration were estimated on the basis of single and/or coexisting mineral chemistry.

Permeability is important in controlling the type and extent of alteration in the studied dike section. At the meter-scale, intervals of weakly altered dolerites containing fresh olivine are interpreted as having experienced restricted exposure to hydrothermal fluids. At the centimeter- or millimeter-scale, alteration patches and extensively altered halos adjacent to veins reflect the permeability related to intergranular primary porosity and cracks. Most of the sheeted dike alteration in this case resulted from non-focused, pervasive fluid-rock interaction.

This study confirms and extends the previous model for hydrothermal alteration at Hole 504B: hydrothermal alteration at the ridge axis followed by seawater recharge and off-axis alteration. The major new discoveries, all related to higher temperatures of alteration, are: (1) the presence of hydrothermal plagioclase $\left(\mathrm{An}_{80-95}\right)$, (2) the presence of deuteric and/or hydrothermal diopside, and (3) the general increasing proportion of amphiboles, and particularly magnesio-hornblende with depth.

We propose that the dolerites at Hole 504B were altered in five stages. Stage 1 occurred at high temperatures (less than $500^{\circ}$ to $700^{\circ} \mathrm{C}$ ) and involved late-magmatic formation of $\mathrm{Na}$ - and Ti-rich diopside, the hydrothermal formation of $\mathrm{Na}$, Ti-poor diopside and the hydrothermal formation of an assemblage of An-rich plagioclase + hornblende. Stage 2 occurred at lower temperatures $\left(250^{\circ}-320^{\circ} \mathrm{C}\right)$ and is characterized by the appearance of actinolite, chlorite, chlorite-smectite, and/or talc (in low permeability zones) and albite. During Stage 3, quartz and epidote precipitated from evolved hydrothermal fluids at temperatures between $310^{\circ}$ and $320^{\circ} \mathrm{C}$. Anhydrite appeared during Stage 4 and likely precipitated directly from heated seawater. Stage 5 occurred off-axis at low temperatures $\left(250^{\circ} \mathrm{C}\right)$ with laumontite and prehnite from evolved fluids.
\end{abstract}

\section{INTRODUCTION}

Deep Sea Drilling Project/Ocean Drilling Program (DSDP/ODP) Hole 504B has come to be regarded as a reference section through the upper oceanic crust (Anderson et al., 1982; Becker, Sakai, et al., 1989). Hole 504B is located in $5.9 \mathrm{Ma}$ crust, $200 \mathrm{~km}$ south of the intermediate-spreading-rate Costa Rica Rift in the eastern Pacific Ocean. The hole was drilled through $274.5 \mathrm{~m}$ of sediment, a $571.5-\mathrm{m}$ volcanic section, a $209-\mathrm{m}$ transition zone, and $945 \mathrm{~m}$ into the sheeted dike complex (Fig. 1). Study of the secondary mineralogy, stable isotopic compositions, and fluid inclusions led to a model for the hydrothermal history of the upper $1500 \mathrm{~m}$ of the crust at Site 504 (Alt et al., 1985, 1986a, b, 1989a, b; Honnorez et al., 1983; Laverne et al., 1989). The volcanic section underwent low temperature $\left(<100^{\circ} \mathrm{C}\right)$ oxidizing alteration at high water/rock ratios $(a t \approx 100)$, whereas the lower volcanics were affected by low temperature alteration under more restricted circulation and more reducing conditions, at lower water/rock ratios $(\approx 10)$. There is an abrupt change to the underlying transition zone and sheeted dikes, which were altered to greenschist facies minerals at higher temperatures $\left(250^{\circ}-380^{\circ} \mathrm{C}\right)$. At the spreading axis, hydrothermal fluids upwelling along fractures in the relatively impermeable dikes mixed with larger volumes of cooler sea-

'Erzinger, J., Becker, K., Dick, H.J.B., and Stokking, L.B. (Eds.), 1995. Proc. ODP, Sci. Results, 137/140: College Station. TX (Ocean Drilling Program).

${ }^{2}$ Laboratoire de Pétrologie Magmatique, URA CNRS 1277 . Faculté des Sciences et Techniques de Saint-Jérôme, Avenue Escadrille Normandie Niemen, 13397 Marseille Cedex 20. France.

${ }^{3}$ Department of Geology, Georgia State University, Atlanta, GA 30303, U.S.A.

${ }^{4}$ Dipartimento di Geologia. Paleontologia e Geofisica, Università di Padova, Via Giotto 1, 35137 Padova, Italy.

${ }^{3}$ Department of Geological Sciences, 1006 C.C. Little Building, University of Michigan, Ann Arbor, MI 48109-1063, U.S.A. water circulating in the more porous and permeable volcanic section, causing the formation of a stockwork-like sulfide mineralization at 900-929 mbsf in the transition zone, and resulting in the formation of a sharp alteration boundary in the crust that coincides with the lithologic boundary. Evolution of hydrothermal fluids resulted in deposition of a sequence of secondary minerals filling fractures in the sheeted dikes: first chlorite and actinolite formed from more seawater-like fluids, followed by epidote, quartz, and sulfides from more evolved fluids. Later recharge of seawater into host rocks led to the precipitation of anhydrite in veins, and finally, off-axis circulation of highly evolved fluids at lower temperatures $\left(<250^{\circ} \mathrm{C}\right)$ resulted in the formation of zeolites in veins and rocks of the upper dike section, and zeolites and carbonates in the volcanics. Trends toward increasing amounts of actinolite, increasing alteration of pyroxene, and decreasing alteration of plagioclase were recognized in samples from Leg 111 (1075.5-1287.5 mbsf), and suggested generally increasing temperatures of alteration downward in the dikes (Alt et al., 1989b).

At a depth of $2000.4 \mathrm{~m}$ below seafloor (mbsf) at the end of Leg 140 , the hole is considered to be very close to the lithologic boundary, which many think separates sheeted dikes of the upper crust from gabbroic rocks in the lower crust. The nature of this boundary is thought to be extremely important in our understanding of how oceanic hydrothermal systems operate. Studies of black smokers typically estimate the depth of the "reaction zone" as $2 \mathrm{~km}$ (e.g., Von Damm et al., 1985), and this is where the source of black smoker fluids might be found. Legs 137 and 140 deepened Hole 504B by drilling $438 \mathrm{~m}$ more in the sheeted dike complex, so that the total thickness of cored sheeted dikes now exceeds $945 \mathrm{~m}$.

This paper documents the mineralogy, petrography, and mineral parageneses associated with secondary alteration of the lower sheeted dikes from Hole 504B drilled during Legs 137/140, to constrain the thermal history and the compositions of hydrothermal fluids. These data are combined and compared with previous results from the upper 


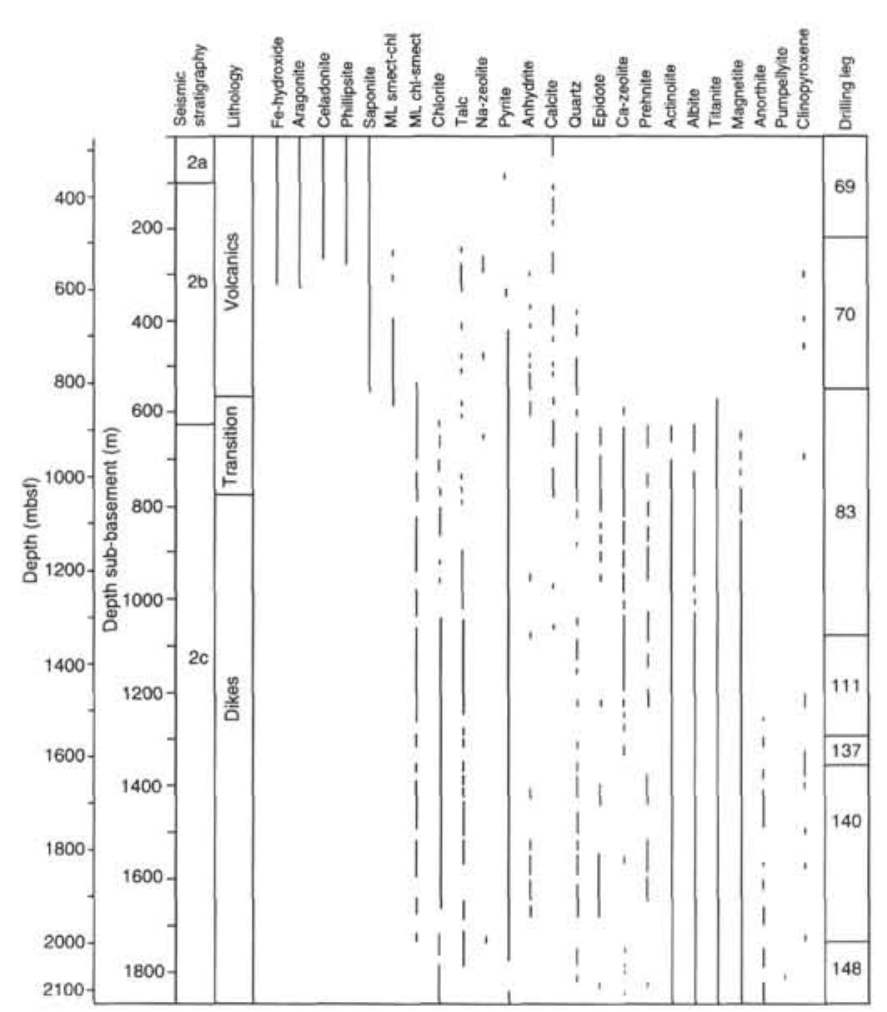

Figure 1. Distribution of secondary minerals with depth in Hole 504B (modified from Shipboard Scientific Party, 1992b). Lithostratigraphy, seismic stratigraphy, and drilling legs also shown (Anderson, Honnorez, Becker, et al., 1985; Shipboard Scientific Party, 1988, 1992a, b). ML smect-chl = mixed-layer smectitechlorite with dominant smectite; $\mathrm{ML} \mathrm{chl}$-smect $=$ mixed-layer chlorite-smectite with dominant chlorite; Na-zeolite includes analcite, natrolite, stilbite, and epistilbite; Ca-zeolite includes laumontite, heulandite, and scolecite.

sheeted dikes in Hole 504B and ophiolites to further constrain hypotheses of hydrothermal alteration of oceanic crust.

\section{METHODS}

Subsequent to shipboard studies, about 250 thin sections from the Legs $137 / 140$ cores were prepared at various institutions. Mineral chemistry was obtained by electron microprobe at Paris $(=P)$ and Brest (= B; France), Padova (= V; Italy), and Atlanta (=A) and Ann Arbor (= AA; USA). All analyses used wavelength dispersive spectrometers and employed well-characterized natural and synthetic standards. Results were processed into mineral chemical formulae using standardized recalculation schemes. Backscattered electron imaging and energy-dispersive $\mathrm{X}$-ray analyses were also used for qualitative chemical analyses and mineral identification.

X-ray diffraction identification of some minerals, as well as preliminary high resolution transmission electron microscope (HRTEM) observations and analyses of secondary phases, were performed in Marseille. Cathodoluminescence microscopy was carried out in Atlanta using a $30 \mathrm{keV}$ cold cathode luminoscope.

Core recovery during Legs $137 / 140$ was approximately $11 \%$; thus the potential for non-representative sampling of the deep sheeted dike section must be kept in mind. The unsampled sections may be randomly distributed, in which case the recovered samples likely are adequately representative. On the other hand, the unsampled sections may be biased toward highly fractured or otherwise crumbly units. The depth of each sample studied is constrained by the known depth of the top and bottom of each cored interval and the position of the sample in the recovered section. In this paper, the sample depths have been calculated using the method proposed by Agrinier and Agrinier (in press). In most plots involving downhole depth, the symbol sizes exceed the depth uncertainty.

\section{PETROGRAPHIC SUMMARY}

Detailed petrographic descriptions of the alteration of this section of dikes are presented in the Leg 140 Initial Reports (Shipboard Scientific Party, 1992b), and are summarized below.

\section{Primary Igneous Features}

Fresh, unaltered dolerite contains plagioclase + clinopyroxene + titanomagnetite \pm olivine \pm spinel, and has a tholeiitic bulk composition. Its grain size varies from aphanitic in chilled margins to as much as $1.9 \mathrm{~mm}$ in the interior of some dikes. There is an overall increase in the maximum grain size with depth, although numerous local variations occur. Olivine has compositions of $\mathrm{Fo}_{86-88}$, primary clinopyroxene may be either $\mathrm{Cr}$-diopside or augite, and primary plagioclase is generally labradorite $\mathrm{An}_{60-75}$. Some recovered dolerites contain sodic plagioclase, quartz, and apatite in interstitial areas, due to late magmatic in-situ crystallization. The dolerite is generally primitive, with $\mathrm{Mg} *\left[\right.$ molar $\left.100 \mathrm{Mg} /\left(\mathrm{Mg}+\mathrm{Fe}^{2+}\right)\right]$ around 65 .

\section{Alteration Features}

Overprinting all dolerite samples is a pervasive background alteration, characterized by $5 \%-40 \%$ replacement of primary minerals by secondary phases. Clinopyroxene is replaced by amphibole, and much more rarely by secondary clinopyroxene. Plagioclase may be replaced by one or more generations of secondary plagioclase, anhydrite, laumontite, epidote, chlorite, or some combination of these minerals. Olivine is variably replaced by chlorite, mixed-layer chlorite-smectite, talc, quartz, sulfides, magnetite, and hematite. Titanomagnetite is replaced by titanite.

In addition to pervasive background alteration, many dolerite samples contain alteration patches and secondary veins with or without adjacent alteration halos. The patches are regions, usually $1-3 \mathrm{~cm}$ in size, but up to about $10 \mathrm{~cm}$ across, of highly altered (up to $100 \%$ ) dolerite. Patches can be spherical, ovoid, or variably elongated: their irregular and variable shapes appear to be independent of veins or any remaining primary features of the dolerite. These patches are apparently zones where hydrothermal solutions had increased access to the dolerite, and were likely initiated within zones of original high primary porosity (e.g., gas vesicles, segregation vesicles, diktytaxitic voids, etc.). Many patches surround $0.1-2 \mathrm{~mm}$ amygdules filled with chlorite and actinolite, and some surround millimeter-scale spherical amygdules with crudely concentric mineral fillings (e.g., actinolite, chlorite, laumontite, and epidote). Larger patches may represent coalescing of smaller patches and continued growth of the alteration zones at the expense of the surrounding dolerite.

Hydrothermal veins, on the order of $1 \mathrm{~mm}$ wide, generally consist of actinolite, chlorite, or actinolite + chlorite, all of which may also contain small amounts of apatite and titanite. Less abundant veins include quartz, epidote, prehnite, laumontite, heulandite (stilbite?), and anhydrite. Detailed descriptions of veins are given in the Leg 140 Initial Reports (Shipboard Scientific Party, 1992b) and in Tartarotti et al. (this volume).

Alteration halos adjacent to veins are usually characterized by extensive recrystallization (typically $40 \%-90 \%$ ). They can be either simple or compound, the latter commonly consisting of a dark interior zone close to the vein and a lighter outer zone. Primary clinopyroxene in the alteration halos is partly replaced by amphibole, and primary plagioclase is partly replaced by one or more generations of secondary plagioclase (anorthite, oligoclase, or albite). Halos also contain accessory secondary titanite, chlorite, and apatite. 


\section{Alteration Distribution}

Whereas there is a general trend toward increasing extent of alteration and increasing proportion of amphibole with depth in the dike section, the extent of alteration varies considerably through the sheeted dikes.

The detailed vein log of Legs $137 / 140$ cores shows that lithologic units with the highest vein abundances are almost invariably associated with chilled margins or fine-grained dolerites (Shipboard Scientific Party, 1992b). This suggests that millimeter-scale fractures, thought to result from thermal contraction, were more prevalent near dike margins. Alteration patches, on the other hand, are actually more common in the coarser grained dike interiors. Both veins and alteration patches occur throughout the Legs 137/140 dike section, showing no apparent depth control.

Unaltered olivine relics are present in rocks from 1705 to 1733 mbsf and sporadically at greater depths, whereas there is only one documented occurrence of fresh olivine in the upper dike section (Becker, Sakai, et al., 1988).

\section{MINERALOGY}

In this section we describe and present chemical data on most major hydrothermal minerals, and we highlight the resulting constraints on the physico-chemical conditions of hydrothermal alteration.

\section{Secondary Clinopyroxene}

Secondary clinopyroxene has been distinguished in seven samples from Legs 137/140. Two types were identified: (1) Ti- and Na-rich diopside-hedenbergite in two samples between 1595 and $1619 \mathrm{mbsf}$, and (2) diopside without anomalous $\mathrm{Ti}$ or $\mathrm{Na}$ contents in five samples from 1573.1 to $1999.1 \mathrm{mbsf}$.

Samples 137-504B-177R-1, 11-15 cm (Piece 4A) and 137-504B$180 \mathrm{M}-1,74-78 \mathrm{~cm}$ (Piece 7) (PI. 1) each contain a 0.2-mm-thick vein composed of actinolite and euhedral titanite, plus chlorite at the center in Sample 137-504B-177R-1, 11-15 cm (Piece 4A). All of the hostrock pyroxene crystals along the vein wall are replaced by a thin $(0.05$ $\mathrm{mm}$ ) layer of bright-green pyroxene (PI. 1). In Sample 137-504B177R-1, 11-15 cm (Piece 4A), this pyroxene changes texture toward the interior of the vein, grading into a colorless, granular form, then colorless and fibrous. Despite the variety of appearances, each of these minerals has a pyroxene stoichiometry. Their high $\mathrm{Na}_{2} \mathrm{O}$ (up to $1.3 \mathrm{wt} \%$ ), $\mathrm{TiO}_{2}$ (up to $1.1 \mathrm{wt} \%$ ) and $\mathrm{Al}_{2} \mathrm{O}_{3}$ (up to $3.3 \mathrm{wt} \%$ ) concentrations (Table 1) clearly distinguish them from primary clinopyroxene (Fig. 2). In contrast to primary clinopyroxene, these secondary pyroxenes contain no $\mathrm{Cr}_{2} \mathrm{O}_{3}$ (Fig. 3). Secondary clinopyroxene from Sample 137-504B-177R-1, 11-15 cm (Piece 4A), is diopside with variable FeO, whereas that from Sample 137-504B-180M-1, 74-78 $\mathrm{cm}$ (Piece 7), varies from diopside to hedenbergite, according to the classification of Morimoto (1988) (Fig. 4). A 30-mm euhedral colorless clinopyroxene crystal occurs within a 0.6 - $\mathrm{mm}$-wide vein of hornblende and actinolite, with chlorite filling the center of the vein in Sample 137-504B-177R-1, 11-14 cm (Piece 4A). This pyroxene does not apparently replace primary clinopyroxene, but it is clearly secondary and exhibits patchy compositional zoning of diopsidic composition (Table 1). Both hornblende and greenish secondary pyroxene also partly replace igneous clinopyroxene along the walls of this vein, although these secondary minerals do not occur in mutual contact.

Diopside, without anomalous $\mathrm{Na}$ concentrations, occurs as very discrete colorless grains recrystallized from igneous clinopyroxene in veins and patches (e.g., Samples 140-504B-189R-2, 100-104 cm (Piece 14), 140-504B-205R-1, 13-16 cm (Piece 1), and 140-504B$237 \mathrm{R}-1,24-26 \mathrm{~cm}$ (Piece 7), or new crystals nucleated among amphibole in veins (Sample 137-504B-176R-1, 15-17 cm (Piece 3). The compositions fall mainly above the line separating "diopside" from "augite" (i.e., $45 \mathrm{~mol} \%$ wollastonite, and for simplicity, we refer to all

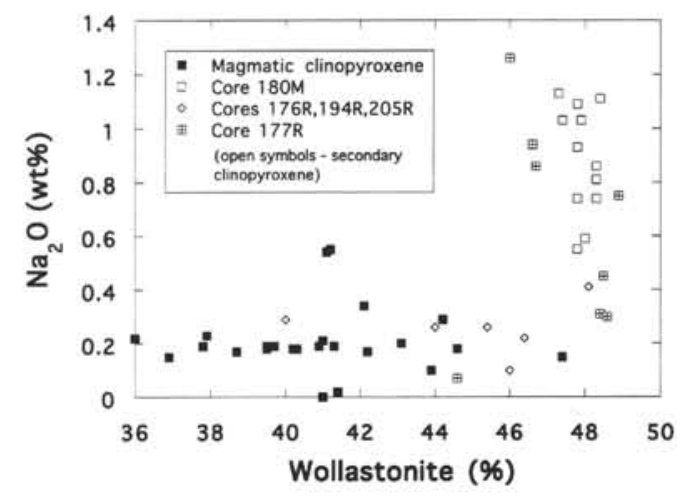

Figure 2. Plot of $\mathrm{Na}_{2} \mathrm{O}$ (wt\%) vs. wollastonite component (mol\%) for selected clinopyroxenes from Hole 504B, Legs 137/140. Most magmatic clinopyroxenes contain less than about $0.4 \mathrm{wt} \% \mathrm{Na}_{2} \mathrm{O}$, whereas hydrothermal clinopyroxenes generally have greater than about $0.4 \% \mathrm{Na}_{2} \mathrm{O}$.

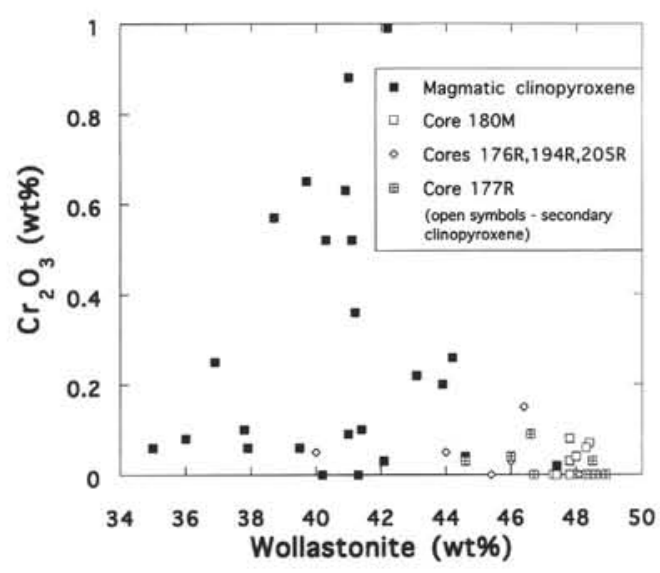

Figure 3. Plot of $\mathrm{Cr}_{2} \mathrm{O}_{3}$ (wt\%) vs. wollastonite component (mol\%) for selected clinopyroxenes from Hole 504B, Legs 137/140. Magmatic clinopyroxenes contain up to about $1.0 \mathrm{wt} \% \mathrm{Cr}_{2} \mathrm{O}_{3}$ at wollastonite compositions generally less than $45 \mathrm{~mol} \%$. Hydrothermal clinopyroxenes, in contrast, invariably have less than $0.2 \mathrm{wt} \% \mathrm{Cr}_{2} \mathrm{O}_{3}$ at wollastonite compositions generally greater than about $45 \mathrm{~mol} \%$.

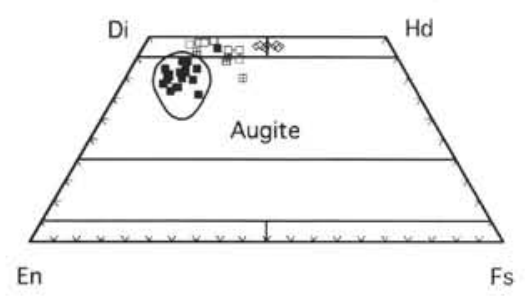

Figure 4. Pyroxene quadrilateral with Hole 504B, Legs 137/140 clinopyroxene compositions (same symbols as Figs. 2 and 3). End-member abbreviations are $\mathrm{Di}=$ diopside, $\mathrm{Hd}=$ hedenbergite, $\mathrm{En}=$ enstatite, and Fs $=$ ferrosilite. The field of magmatic clinopyroxene (after McNeill, this volume) is represented.

of these as "diopside"). Colorless fine-grained diopside, associated with scarce $\mathrm{Mg}$-hornblende, also fills a $0.4-\mathrm{mm}$-thick vein in Sample 140-504B-213R-1, 3-6 cm (Piece 2). The diopside is enriched in Fe and $\mathrm{Ca}$ relative to primary augite (Fig. 4) and contains no detectable $\mathrm{Cr}_{2} \mathrm{O}_{3}$, in contrast to fresh augite (Fig. 3).

\section{Amphibole}

Amphibole is widespread throughout the Legs $137 / 140$ section. It occurs as an alteration product of igneous clinopyroxene and filling 
Table 1. Representative secondary clinopyroxene analyses, Hole 504B, Legs 137/140.

\begin{tabular}{|c|c|c|c|c|c|c|c|c|c|c|c|c|c|c|c|c|}
\hline $\begin{array}{l}\text { Core, section, } \\
\text { interval }(\mathrm{cm})\end{array}$ & Analysis & Color & Occurrence & $\mathrm{SiO}_{2}$ & $\mathrm{TiO}_{2}$ & $\mathrm{Al}_{2} \mathrm{O}_{3}$ & $\mathrm{FeO}$ & $\mathrm{MnO}$ & $\mathrm{MgO}$ & $\mathrm{CaO}$ & $\mathrm{Na}_{2} \mathrm{O}$ & $\mathrm{K}_{2} \mathrm{O}$ & $\mathrm{Cr}_{2} \mathrm{O}_{3}$ & $\mathrm{Cl}$ & $\mathrm{ZnO}$ & Total \\
\hline $173 \mathrm{R}-1,77-79$ & B2185 & clss & $\mathrm{m}$ & 52.71 & 0.14 & 0.78 & 11.63 & 0.26 & 12.73 & 21.75 & 0.18 & 0.01 & 0.04 & 0.00 & 0.00 & 100.23 \\
\hline $176 \mathrm{R}-1,15-17$ & A7 & clss & amp vein cent & 50.46 & 0.09 & 0.87 & 17.55 & 0.15 & 8.13 & 23.28 & 0.41 & 0.00 & 0.00 & nd & nd & 100.94 \\
\hline $177 \mathrm{R}-1,11-14$ & AA1 & clss & amp vein cent & 51.95 & 0.04 & 0.18 & 7.82 & 0.13 & 14.42 & 24.78 & 0.45 & 0.01 & 0.00 & nd & nd & 99.78 \\
\hline \multirow{4}{*}{$177 \mathrm{R}-1,11-15$} & B2109 & gr & amp vein wall & 52.87 & 0.05 & 0.77 & 13.29 & 0.25 & 11.76 & 21.67 & 0.07 & 0.00 & 0.03 & 0.03 & 0.18 & 100.97 \\
\hline & B2112 & $\mathrm{gr}$ & amp vein wall & 54.58 & 0.21 & 0.20 & 6.99 & 0.17 & 14.59 & 24.44 & 0.30 & 0.02 & 0.00 & 0.00 & 0.00 & 101.50 \\
\hline & B2114 & $\mathrm{gr}$ & amp vein wall & 51.12 & 1.15 & 1.50 & 12.25 & 0.15 & 11.59 & 21.96 & 1.26 & 0.00 & 0.04 & 0.00 & 0.00 & 101.02 \\
\hline & B2122 & $\mathrm{gr}$ & amp vein wall & 51.08 & 0.79 & 1.01 & 12.50 & 0.28 & 11.40 & 22.65 & 0.86 & 0.00 & 0.00 & 0.00 & 0.00 & 100.57 \\
\hline \multirow{11}{*}{$180 \mathrm{M}-1,74-78$} & B5061 & clss & $\mathrm{m}$ & 53.02 & 0.28 & 2.79 & 5.34 & 0.28 & 18.38 & 19.68 & 0.19 & 0.00 & 0.65 & nd & nd & 100.62 \\
\hline & B5062 & clss & $\mathrm{m}$ & 53.85 & 0.17 & 2.44 & 5.38 & 0.09 & 18.69 & 19.08 & 0.17 & 0.02 & 0.57 & nd & nd & 100.47 \\
\hline & B5063 & gr & amp vein wall & 49.31 & 0.29 & 2.66 & 16.33 & 0.28 & 8.26 & 22.37 & 0.93 & 0.00 & 0.00 & nd & nd & 100.42 \\
\hline & B5064 & $\mathrm{gr}$ & amp vein wall & 51.27 & 0.14 & 1.10 & 14.52 & 0.31 & 10.00 & 23.30 & 0.55 & 0.01 & 0.03 & nd & nd & 101.22 \\
\hline & B5065 & $\mathrm{gr}$ & amp vein wall & 49.50 & 0.44 & 2.73 & 14.60 & 0.10 & 9.15 & 22.21 & 1.03 & 0.00 & 0.00 & nd & nd & 99.76 \\
\hline & B5071 & $\mathrm{gr}$ & amp vein wall & 50.96 & 0.07 & 1.18 & 14.84 & 0.34 & 9.44 & 22.91 & 0.74 & 0.00 & 0.00 & nd & nd & 100.49 \\
\hline & B5072 & $\mathrm{gr}$ & amp vein wall & 50.17 & 0.32 & 1.89 & 15.14 & 0.41 & 8.76 & 22.74 & 0.74 & 0.00 & 0.00 & nd & nd & 100.16 \\
\hline & B5075 & $\mathrm{gr}$ & amp vein wall & 48.15 & 0.60 & 3.33 & 16.13 & 0.23 & 7.77 & 22.10 & 1.11 & 0.00 & 0.07 & nd & nd & 99.49 \\
\hline & B5077 & $\mathrm{gr}_{\mathrm{gr}} \mathrm{r}$ & amp vein wall & 48.76 & 0.22 & 2.85 & 16.62 & 0.40 & 7.80 & 22.08 & 1.09 & 0.05 & 0.08 & nd & nd & 99.94 \\
\hline & B5079 & $\mathrm{gr}$ & amp vein wall & 48.51 & 0.42 & 3.12 & 16.68 & 0.19 & 7.94 & 21.81 & 1.03 & 0.01 & 0.00 & nd & nd & 99.71 \\
\hline & B5080 & $\mathrm{gr}$ & amp vein wall & 50.04 & 0.25 & 1.66 & 15.07 & 0.35 & 8.69 & 22.53 & 0.86 & 0.00 & 0.06 & nd & nd & 99.52 \\
\hline $189 \mathrm{R}-1,105-106$ & B2016 & clss & $\mathrm{m}$ & 52.83 & 0.35 & 2.65 & 6.67 & 0.17 & 18.33 & 18.03 & 0.15 & 0.00 & 0.25 & 0.00 & 0.13 & 99.56 \\
\hline $189 \mathrm{R}-2,76-79$ & B2164 & clss & $\mathrm{m}$ & 52.49 & 0.34 & 2.84 & 5.05 & 0.25 & 18.00 & 20.28 & 0.19 & 0.02 & 0.63 & 0.01 & 0.00 & 100.10 \\
\hline \multirow{2}{*}{$189 \mathrm{R}-2,100-104$} & A47 & clss & amp+chl patch & 51.86 & 0.05 & 0.32 & 11.90 & 0.31 & 12.98 & 21.68 & 0.26 & 0.02 & 0.05 & nd & nd & 99.43 \\
\hline & A52 & clss & $\mathrm{m}$ & 51.16 & 0.40 & 2.70 & 0.20 & 5.97 & 0.15 & 16.26 & 21.42 & 0.10 & 0.00 & nd & nd & 98.36 \\
\hline $200 \mathrm{R}-2,101-104$ & B2104 & clss & il & 51.73 & 0.63 & 3.65 & 7.05 & 0.08 & $\begin{array}{r}0.15 \\
16.09\end{array}$ & 21.15 & $\begin{array}{r}2.42 \\
0.20\end{array}$ & 0.00 & 0.22 & 0.00 & 0.00 & 100.80 \\
\hline $205 \mathrm{R}-1,13-16$ & A68 & clss & recr in halo & 52.23 & 0.21 & 1.59 & 7.29 & 0.27 & 14.89 & 23.08 & 0.22 & 0.00 & 0.15 & nd & nd & 99.93 \\
\hline \multirow{2}{*}{$212 R-1,61-65$} & B5094 & clss & $\mathrm{m}$ & 51.54 & 0.51 & 3.08 & 7.37 & 0.24 & 16.48 & 20.31 & 0.19 & 0.00 & 0.00 & 0.00 & 0.00 & 99.72 \\
\hline & B5092 & clss & $\mathrm{m}$ & 51.99 & 0.53 & 2.74 & 0.06 & 8.78 & 0.38 & 16.33 & 19.50 & 0.18 & 0.00 & nd & nd & 100.49 \\
\hline \multirow[t]{2}{*}{$213 R-1,3-6$} & V1023 & clss & vein $(a$ & 53.51 & 0.17 & 1.90 & 14.30 & 0.38 & 13.82 & 16.11 & 0.32 & 0.03 & 0.02 & nd & nd & 100.56 \\
\hline & V1029 & clss & vein (alone) & 53.41 & 0.18 & 0.70 & 12.46 & 0.39 & 13.91 & 18.75 & 0.14 & 0.01 & 0.00 & nd & nd & 99.95 \\
\hline $225 \mathrm{R}-1,126-129$ & B5042 & clss & $\mathrm{m}$ & 51.99 & 0.53 & 2.74 & 8.78 & 0.38 & 16.33 & 19.50 & 0.19 & 0.00 & 0.06 & 0.05 & 0.00 & 100.55 \\
\hline & B2072 & clss & $\mathrm{m}$ & 49.64 & 1.05 & 1.63 & 18.55 & 0.61 & 11.74 & 16.84 & 0.53 & 0.12 & 0.06 & 0.18 & 0.00 & 100.95 \\
\hline \multirow{2}{*}{$237 \mathrm{R}-1,24-26$} & B3008 & clss & $\mathrm{m}$ & 51.99 & 0.45 & 3.31 & 7.20 & 0.20 & 17.88 & 18.71 & 0.23 & 0.04 & 0.06 & 0.00 & 0.00 & 100.07 \\
\hline & Al05 & clss & recr in halo & 52.65 & 0.00 & 0.57 & 11.64 & 0.40 & 11.56 & 21.71 & 0.10 & 0.00 & 0.03 & nd & nd & 98.66 \\
\hline
\end{tabular}

Notes: clss $=$ colorless; $\mathrm{gr}=$ green; amp $=$ amphibole; chl $=$ chlorite; $\mathrm{m}=$ magmatic; recr $=$ recrystallized; $\mathrm{nd}=$ not determined. The projected Wo'-En $-\mathrm{Fs}^{\prime}-\mathrm{Ac}-\mathrm{Jd}-\mathrm{FeCaTs}-\mathrm{CrCaTs}^{\prime}-\mathrm{AlCaTs}$ values have been calculated according to Lindsley and Anderson (1983). 
Table 1 (continued).

\begin{tabular}{|c|c|c|c|c|c|c|c|c|c|c|c|c|c|c|c|c|c|c|c|}
\hline $\begin{array}{l}\text { Core, section, } \\
\text { interval }(\mathrm{cm})\end{array}$ & $\mathrm{Si}$ & $\mathrm{Ti}$ & $\mathrm{Al}$ & $\mathrm{Cr}$ & $\mathrm{FeT}$ & $\mathrm{Fe}^{3+}$ & $\mathrm{Fe}^{2+}$ & $\mathrm{Mn}$ & $\mathrm{Mg}$ & $\mathrm{Ca}$ & $\mathrm{Na}$ & K & Total & Wo & En & Fs & Wo' & $\mathrm{En}^{\prime}$ & $\mathrm{Fs}^{\prime}$ \\
\hline $173 \mathrm{R}-1,77-79$ & 1.983 & 0.004 & 0.035 & 0.001 & 0.366 & 0.003 & 0.363 & 0.008 & 0.714 & 0.877 & 0.013 & 0.000 & 4.001 & 44.6 & 36.3 & 19.0 & 43.6 & 37.4 & 19.0 \\
\hline $176 \mathrm{R}-1,15-17$ & 1.943 & 0.003 & 0.039 & 0.000 & 0.565 & 0.075 & 0.490 & 0.005 & 0.467 & 0.960 & 0.031 & 0.000 & 4.013 & 48.1 & 23.4 & 28.5 & 46.1 & 27.0 & 26.9 \\
\hline $177 \mathrm{R}-1,11-14$ & 1.937 & 0.001 & 0.008 & 0.001 & 0.244 & 0.111 & 0.133 & 0.004 & 0.801 & 0.990 & 0.033 & 0.000 & 4.019 & 48.5 & 39.3 & 12.2 & 45.3 & 48.8 & 5.8 \\
\hline \multirow{4}{*}{$\begin{array}{l}177 \mathrm{R}-1,11-15 \\
115\end{array}$} & 1.993 & $\begin{array}{l}0.001 \\
0.001\end{array}$ & 0.034 & 0.001 & $\begin{array}{l}0.244 \\
0.419\end{array}$ & $\begin{array}{l}0.014 \\
-0.014\end{array}$ & 0.433 & $\begin{array}{l}0.004 \\
0.008\end{array}$ & $\begin{array}{l}0.801 \\
0.661\end{array}$ & 0.875 & $\begin{array}{l}0.033 \\
0.005\end{array}$ & 0.000 & $\begin{array}{l}4.019 \\
3.998\end{array}$ & 44.6 & 33.7 & 21.8 & $\begin{array}{l}43.3 \\
42.6\end{array}$ & $\begin{array}{l}48.8 \\
35.1\end{array}$ & 22.3 \\
\hline & $\begin{array}{l}1.993 \\
1.995\end{array}$ & $\begin{array}{l}0.001 \\
0.006\end{array}$ & $\begin{array}{l}0.034 \\
0.009\end{array}$ & $\begin{array}{l}0.001 \\
0.000\end{array}$ & $\begin{array}{l}0.419 \\
0.214\end{array}$ & 0.009 & $\begin{array}{l}0.433 \\
0.205\end{array}$ & $\begin{array}{l}0.008 \\
0.005\end{array}$ & $\begin{array}{l}0.001 \\
0.795\end{array}$ & 0.957 & $\begin{array}{l}0.000 \\
0.021\end{array}$ & 0.001 & $\begin{array}{l}3.998 \\
4.002\end{array}$ & $\begin{array}{l}48.0 \\
48.6\end{array}$ & 40.3 & 11.1 & $\begin{array}{l}42.0 \\
48.4\end{array}$ & $\begin{array}{l}30.1 \\
41.1\end{array}$ & 10.4 \\
\hline & 1.912 & 0.032 & 0.066 & 0.001 & 0.383 & 0.102 & 0.282 & 0.005 & 0.646 & 0.880 & 0.091 & 0.000 & 4.017 & 46.0 & 33.8 & 20.3 & 46.3 & 38.8 & 14.9 \\
\hline & 1.926 & 0.022 & 0.045 & 0.000 & 0.394 & 0.091 & 0.303 & 0.009 & 0.641 & 0.915 & 0.063 & 0.000 & 4.015 & 46.7 & 32.7 & 20.6 & 46.0 & 37.9 & 16.1 \\
\hline \multirow[t]{11}{*}{$180 \mathrm{M}-1,74-78$} & 1.919 & 0.008 & 0.119 & 0.019 & 0.162 & 0.017 & 0.145 & 0.009 & 0.992 & 0.763 & 0.013 & 0.000 & 4.003 & 39.7 & 51.5 & 8.8 & 35.5 & 56.5 & 7.9 \\
\hline & 1.948 & 0.005 & 0.104 & 0.016 & 0.163 & -0.010 & 0.173 & 0.003 & 1.008 & 0.740 & 0.012 & 0.001 & 3.999 & 38.7 & 52.7 & $\begin{array}{l}8.0 \\
8.7\end{array}$ & 34.2 & 56.7 & 9.2 \\
\hline & 1.893 & 0.008 & 0.120 & 0.000 & 0.524 & 0.109 & 0.416 & 0.009 & 0.473 & 0.920 & 0.069 & 0.000 & 4.018 & 47.8 & 24.5 & 27.7 & 45.0 & 30.5 & 24.5 \\
\hline & 1.940 & 0.004 & 0.049 & 0.001 & 0.460 & 0.077 & 0.383 & 0.010 & 0.564 & 0.945 & 0.040 & 0.000 & 4.013 & 47.8 & 28.5 & 23.7 & 46.1 & 33.0 & 20.9 \\
\hline & 1.898 & 0.013 & 0.123 & 0.000 & 0.468 & 0.099 & 0.369 & 0.003 & 0.523 & 0.912 & 0.077 & 0.000 & 4.017 & 47.9 & 27.4 & 24.7 & 45.6 & 33.1 & 21.3 \\
\hline & 1.945 & 0.002 & 0.053 & 0.000 & 0.474 & 0.081 & 0.393 & 0.011 & 0.537 & 0.937 & 0.055 & 0.000 & 4.013 & 47.8 & 27.4 & 24.7 & 46.9 & 31.6 & 21.5 \\
\hline & 1.926 & 0.009 & 0.086 & 0.000 & 0.486 & 0.074 & 0.412 & 0.013 & 0.501 & 0.936 & 0.055 & 0.000 & 4.012 & 48.3 & 25.9 & 25.8 & 46.8 & 30.0 & 23.2 \\
\hline & 1.867 & 0.017 & 0.152 & 0.002 & 0.523 & 0.120 & 0.403 & 0.008 & 0.449 & 0.918 & 0.083 & 0.000 & 4.020 & 48.4 & 23.7 & 28.0 & 45.2 & 30.3 & 24.5 \\
\hline & 1.885 & 0.006 & 0.130 & 0.002 & 0.537 & 0.125 & 0.412 & 0.013 & 0.449 & 0.915 & 0.082 & 0.002 & 4.022 & 47.8 & 23.5 & 28.8 & 447 & 30.3 & 250 \\
\hline & 1.878 & 0.012 & 0.142 & 0.000 & 0.540 & 0.116 & 0.424 & 0.006 & 0.458 & 0.905 & 0.077 & 0.000 & 4.019 & 47.4 & 24.0 & 28.6 & 44.2 & 30.3 & 25.5 \\
\hline & 1.933 & 0.007 & 0.076 & 0.002 & 0.487 & 0.080 & 0.407 & 0.011 & $\begin{array}{l}0.430 \\
0.500\end{array}$ & 0.933 & 0.064 & 0.000 & 4.013 & $\begin{array}{l}47.4 \\
48.3\end{array}$ & 25.9 & 258 & 47.2 & 30.3 & 22.8 \\
\hline $189 \mathrm{R}-1,105-106$ & $\begin{array}{l}1.935 \\
1.937\end{array}$ & $\begin{array}{l}0.001 \\
0.010\end{array}$ & $\begin{array}{l}0.076 \\
0.115\end{array}$ & $\begin{array}{l}0.002 \\
0.007\end{array}$ & $\begin{array}{l}0.481 \\
0.205\end{array}$ & $\begin{array}{r}0.000 \\
-0.004\end{array}$ & $\begin{array}{l}0.407 \\
0.208\end{array}$ & $\begin{array}{l}0.011 \\
0.005\end{array}$ & $\begin{array}{l}0.500 \\
1.002\end{array}$ & $\begin{array}{l}0.933 \\
0.708\end{array}$ & $\begin{array}{l}0.004 \\
0.011\end{array}$ & 0.000 & $\begin{array}{l}4.013 \\
3.999\end{array}$ & $\begin{array}{l}48.3 \\
36.9\end{array}$ & 52.2 & $\begin{array}{l}2.8 \\
10.9\end{array}$ & $\begin{array}{l}47.2 \\
33.0\end{array}$ & $\begin{array}{l}30.0 \\
55.6\end{array}$ & $\begin{array}{l}22.8 \\
11.4\end{array}$ \\
\hline $\begin{array}{l}189 \mathrm{~K}-1,10-100 \\
189 \mathrm{R}-2,76-79\end{array}$ & $\begin{array}{l}1.937 \\
1.909\end{array}$ & $\begin{array}{l}0.010 \\
0.009\end{array}$ & 0.122 & $\begin{array}{l}0.007 \\
0.018\end{array}$ & $\begin{array}{l}0.203 \\
0.160\end{array}$ & $\begin{array}{r}-0.004 \\
0.028\end{array}$ & $\begin{array}{l}0.208 \\
0.131\end{array}$ & $\begin{array}{l}0.003 \\
0.008\end{array}$ & $\begin{array}{l}1.002 \\
0.975\end{array}$ & $\begin{array}{l}0.790 \\
0.790\end{array}$ & $\begin{array}{l}0.011 \\
0.013\end{array}$ & 0.001 & $\begin{array}{l}3.999 \\
4.005\end{array}$ & $\begin{array}{l}36.9 \\
40.9\end{array}$ & $\begin{array}{l}32.2 \\
50.5\end{array}$ & $\begin{array}{r}10.9 \\
8.7\end{array}$ & $\begin{array}{l}33.0 \\
36.5\end{array}$ & $\begin{array}{l}33.0 \\
56.4\end{array}$ & $\begin{array}{r}11.4 \\
7.1\end{array}$ \\
\hline \multirow{2}{*}{$189 \mathrm{R}-2,100-104$} & 1.968 & 0.001 & 0.014 & 0.001 & 0.378 & 0.048 & 0.329 & 0.010 & 0.734 & 0.882 & $\begin{array}{l}0.019 \\
0.019\end{array}$ & 0.001 & $\begin{array}{l}4.0009 \\
4.009\end{array}$ & 44.0 & 36.6 & $\begin{array}{l}8.1 \\
19.4\end{array}$ & $\begin{array}{l}30.3 \\
42.7\end{array}$ & $\begin{array}{l}30.4 \\
40.2\end{array}$ & 17.1 \\
\hline & $\begin{array}{l}1.908 \\
1.910\end{array}$ & 0.011 & $\begin{array}{l}0.014 \\
0.119\end{array}$ & $\begin{array}{l}0.001 \\
0.006\end{array}$ & 0.186 & $\begin{array}{l}0.031 \\
0.031\end{array}$ & $\begin{array}{l}0.329 \\
0.156\end{array}$ & 0.005 & $\begin{array}{l}0.905 \\
0.905\end{array}$ & $\begin{array}{l}0.882 \\
0.857\end{array}$ & 0.007 & 0.000 & 4.005 & 43.9 & 46.3 & 9.8 & 39.8 & 51.9 & 8.3 \\
\hline $200 \mathrm{R}-2,101-104$ & 1.889 & 0.017 & 0.157 & 0.006 & 0.215 & 0.029 & 0.187 & 0.002 & 0.876 & 0.828 & 0.014 & 0.000 & 4.005 & 43.1 & $\begin{array}{l}40.5 \\
45.6\end{array}$ & 11.3 & 38.3 & 51.4 & 10.4 \\
\hline $205 \mathrm{R}-1,13-16$ & 1.937 & 0.006 & 0.070 & 0.004 & 0.226 & 0.042 & 0.184 & 0.008 & 0.823 & 0.917 & 0.016 & 0.000 & 4.007 & $\begin{array}{l}43.1 \\
46.4\end{array}$ & $\begin{array}{l}43.0 \\
41.7\end{array}$ & 11.9 & 44.1 & $\begin{array}{l}31.4 \\
46.4\end{array}$ & $\begin{array}{r}10.4 \\
9.6\end{array}$ \\
\hline \multirow{2}{*}{$212 \mathrm{R}-1,61-65$} & 1.878 & 0.014 & 0.132 & 0.000 & 0.225 & 0.072 & 0.153 & 0.007 & 0.949 & 0.793 & 0.013 & 0.000 & 4.012 & 40.2 & 48.1 & 11.8 & 35.6 & 56.7 & 7.7 \\
\hline & 1.912 & 0.015 & 0.119 & 0.002 & 0.270 & 0.030 & 0.240 & 0.012 & 0.895 & 0.768 & 0.013 & 0.000 & 4.005 & 39.5 & 46.0 & 14.5 & 36.1 & 50.8 & 13.1 \\
\hline \multirow{2}{*}{$213 R-1,3-6$} & 2.002 & 0.005 & 0.084 & 0.001 & 0.447 & -0.056 & 0.503 & 0.012 & 0.771 & 0.646 & 0.023 & 0.001 & 3.991 & 34.4 & 41.1 & 24.5 & 29.1 & 44.8 & 26.0 \\
\hline & 2.008 & 0.005 & 0.031 & 0.000 & 0.392 & -0.036 & 0.428 & 0.012 & 0.779 & 0.755 & 0.010 & 0.000 & 3.994 & 39.0 & 40.2 & 20.8 & 36.3 & 42.4 & 21.3 \\
\hline $225 \mathrm{R}-1,126-129$ & 1.912 & 0.015 & 0.119 & 0.002 & 0.270 & 0.031 & 0.239 & 0.012 & 0.895 & 0.768 & 0.014 & 0.000 & 4.005 & 39.5 & 46.0 & 14.5 & 36.1 & 50.8 & 13.0 \\
\hline $226 \mathrm{R}-1,99-102$ & 1896 & 0.030 & 0.073 & 0.002 & 0.593 & 0.083 & 0.509 & 0.020 & 0.668 & 0.689 & 0.039 & 0.006 & 4.017 & 35.0 & 33.9 & 311 & 32.7 & 39.1 & 28.2 \\
\hline \multirow[t]{2}{*}{$237 \mathrm{R}-1,24-26$} & $\begin{array}{l}1.070 \\
1.900\end{array}$ & 0.012 & 0.143 & $\begin{array}{l}0.002 \\
0.002\end{array}$ & 0.220 & 0.036 & 0.184 & $\begin{array}{l}0.020 \\
0.006\end{array}$ & $\begin{array}{l}0.070 \\
0.974\end{array}$ & 0.733 & 0.016 & 0.002 & $\begin{array}{l}4.007 \\
4.007\end{array}$ & 37.9 & 50.4 & 11.7 & 33.7 & 56.4 & $\begin{array}{l}20.2 \\
10.0\end{array}$ \\
\hline & 2.019 & 0.000 & 0.026 & $\begin{array}{l}0.002 \\
0.001\end{array}$ & $\begin{array}{l}0.220 \\
0.373\end{array}$ & $\begin{array}{l}0.0043 \\
-0.043\end{array}$ & $\begin{array}{l}0.184 \\
0.417\end{array}$ & $\begin{array}{l}0.000 \\
0.013\end{array}$ & $\begin{array}{l}0.974 \\
0.661\end{array}$ & 0.892 & $\begin{array}{l}0.010 \\
0.007\end{array}$ & 0.000 & $\begin{array}{l}4.001 \\
3.993\end{array}$ & 46.0 & $\begin{array}{l}34.4 \\
34.1\end{array}$ & 19.9 & 42.7 & $\begin{array}{l}30.4 \\
36.6\end{array}$ & $\begin{array}{l}10.0 \\
20.7\end{array}$ \\
\hline
\end{tabular}


vugs, patches and veins (Pl. 1). Amphibole also occurs in interstitial groundmass areas, where no clear relationships to primary clinopyroxene can be identified.

Amphibole replacing clinopyroxene generally occurs as pseudomorphs or fine-grained fibers growing from the pyroxene crystal rims. In alteration patches amphibole is usually associated with chlorite, albite, titanite ( \pm epidote, anhydrite and prehnite). Amphibole may also fill amygdules along with chlorite (Shipboard Scientific Party, 1992b).

Amphibole in veins occurs as both fine fibers and prismatic crystals. It may be associated with chlorite, laumontite, titanite, or apatite in compositionally complex veins. In a few veins, aggregates of needle-shaped amphibole have been observed, usually associated with laumontite. The vein-filling fibers exhibit no preferred shape orientation, although their elongation direction usually forms a high angle with the vein edges. These fibers are frequently deformed by kinking or bending. Along the vein walls, amphibole orientation is controlled by the cleavage orientation of clinopyroxene in the wallrock. Amphibole along vein walls partly replaces clinopyroxene, and it usually occurs as syntaxial overgrowths on pyroxene. In a few examples, more than one opening stage can be inferred from the textural arrangement of the vein-filling minerals (Tartarotti et al., this volume).

About 360 analyses of amphibole have been conducted by electron microprobe on the Legs $137 / 140$ samples, and representative analyses are reported in Table 2. Structural formulas of amphibole and the partitioning iron were determined using the AMPHIBOL.EXE program (D. Hermitte, pers. comm., 1992), which assumes 15 total cations per formula unit, excluding potassium, and partitions ferrous and ferric iron to satisfy charge balance.

Following the nomenclature of Leake (1978), amphiboles have been classified into actinolite, Fe-actinolite, actinolitic hornblende, magnesio hornblende, edenite, and cummingtonite. Table 3 shows that most $(60 \%)$ amphibole in the center and walls of veins is actinolite or Fe-actinolite, while most (70\%) amphibole in the halos around veins is act-hornblende or mg-hornblende. Although not shown in Table 3, composite veins are characterized by actinolite in the center and hornblende along the walls. The groundmass away from veins and alteration halos is characterized by almost equal proportions of actinolite (actinolite $+\mathrm{Fe}$-actinolite $=41 \%$ ) and hornblende (acthornblende $+\mathrm{mg}$-hornblende $=44 \%$ ). Amphibole in alteration halos around veins is locally iron-rich (Fe-actinolite; Table 2), whereas amphibole in the adjacent veins does not exhibit Fe-enrichment. Amphibole replacing clinopyroxene both in the halos and in the groundmass away from halos frequently exhibits higher $\mathrm{Cr}_{2} \mathrm{O}_{3}$ contents than does amphibole filling veins and patches (Table 2). The amphiboles generally have low chlorine concentration ranging from 0 to $0.10 \mathrm{wt} \% \mathrm{Cl}$, with a maximum of about $0.5 \mathrm{wt} \% \mathrm{Cl}$.

The maximum $\mathrm{Al}$ concentration at any depth is very irregular in the Leg 140 section, though occurrences of higher- $\mathrm{Al}$ amphiboles are more frequent than in the overlying dikes. The minimum Al concentration as a function of depth increases smoothly in the Legs 137/140 section, with the exception of the interval 1980-1990 mbsf, where low Al-amphiboles occur (Fig. 5).

The variation in amphibole composition with textural occurrence in the dolerite samples and the lack of major systematic downhole variation suggest that rock alteration is very heterogeneous in this section of the sheeted dike complex. One possibility is that amphibole composition has changed during the superposition of alteration stages occurring under different temperatures and/or different fluid compositions. If temperature was the controlling parameter, $\mathrm{Mg}$-hornblende and actinolitic hornblende (associated with secondary Ca-plagioclase) formed in veins and in alteration halos around veins during early higher temperature hydrothermal alteration. Reactions may have been restricted to the vein and halo with little alteration of the host rock because the stage was too short-lived or because not enough fluid was available to react with the host rock. Halo development may have been restricted because of a generally low rock permeability. Subsequently, as the system cooled, actinolite formed pervasively throughout the rock and in veins, whereas the previously formed hornblende was relatively stable so it did not react. Progressively cooling conditions are corroborated by the occurrence of laumontite filling the interstitial spaces in actinolite veins (Sample 140-504B-186R-1, 57-60 cm, Piece 10) and in actinolite-filled pore spaces in some alteration patches. Amphibole composition was also probably affected by fluid composition which may account for the local $\mathrm{Fe}$-enrichment. $\mathrm{Cr}$-enrichment in amphibole replacing clinopyroxene may be explained by the fact that amphibole inherited the composition of the original mineral phase (Tartarotti et al., this volume). Rock alteration is clearly very heterogeneous as a function of depth. The occurrence of high $\mathrm{Al}^{\mathrm{IV}}$ contents accompanied by high $\mathrm{TiO}_{2}$ contents in amphibole at specific depth values suggests that probably higher temperatures of equilibration were locally reached (Liou et al., 1974; Blundy and Holland, 1990).

\section{Secondary Calcic Plagioclase, Albite, and Oligoclase}

Secondary calcic plagioclase occurs in some extensively altered vein halos as incomplete replacement around the edges of primary igneous plagioclase laths, or total replacement of smaller laths enclosed in amphibole. The rims are compositionally distinct based upon differences in extinction angle, and they are charged with 10$\mathrm{mm}$-scale aqueous fluid inclusions.

Variations in plagioclase compositions (Table 4) and the textures of replacement are particularly easily observed with cathodoluminescence (abbreviated as CL hereafter; Mora and Ramseyer, 1992). Most vein halos are characterized by distinctly luminescing yellowishgreen rims of secondary calcic plagioclase on the primary igneous plagioclase laths (Pl. 2). Electron microprobe analyses indicate that the primary plagioclase is typically labradorite, and that the fluidinclusion-filled rims, typically bytownite and anorthite (Fig. 6), are uniformly more calcic than the cores.

Calcic plagioclase replacement rims are also chemically distinct from primary labradorite cores in minor elements. The most striking distinction is in $\mathrm{MgO}$ concentration, which is always lower in secondary plagioclase (Fig. 6). The concentration of $\mathrm{FeO}$ is also notably lower in secondary plagioclase.

In some vein halos, in many patches and in some rocks exhibiting strong background alteration, primary plagioclase laths are altered along microcracks to albite or oligoclase (Table 4). Secondary albite is characterized by little or no CL, thus it stands out in contrast to both the dull-CL primary plagioclase laths and the distinct-CL rims, both of which are cut by the albite when present. Fluid inclusions in albite are present but not as abundant as in the calcic rims. Other phases that also form vein-like replacement along fractures within plagioclase include laumontite, which has a dull reddish-orange CL color, and chlorite. These minerals cut both primary plagioclase and calcic rims, thus they appear to be related temporally to secondary albite. This is consistent with the occurrence of albite with laumontite and chlorite in patches of alteration interstitial to relict igneous plagioclase.

The occurrence of hydrothermal plagioclase that is more calcic than the associated igneous plagioclase has been recognized here for the first time in Hole 504B and, to our knowledge, for the first time in any oceanic rock. Calcic secondary plagioclase occurs in numerous cores between Cores 137-504B-174R and -229R. It has been positively identified at depths of 1577-1600, 1621, 1628, 1652, 1662, 1681, $1702-1760,1778,1795,1818,1828,1875-1893$, and 1912-1950 mbsf. Re-examination of a set of thin sections from Leg 111 reveals only one occurrence of calcic plagioclase rims, in Core 162R at a depth of approximately $1500 \mathrm{mbsf}$. The appearance of calcic plagioclase downhole correlates roughly with increases in, but not the first appearance of, both hydrothermal clinopyroxene and magnesio-hornblende.

\section{Chlorite and Chlorite-Smectite}

Chlorite occurs in veins, either alone or associated with actinolite. Common composite chlorite-actinolite veins are commonly com- 


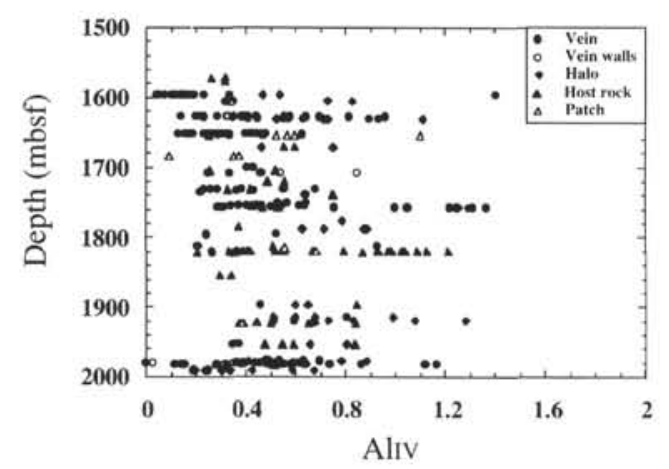

Figure 5. Plot of $\mathrm{Al}^{\mathrm{IV}}$ vs. depth for amphiboles from Hole 504B, Legs 137/140. Note the broad trend of slightly higher $\mathrm{Al}^{\mathrm{IV}}$ with depth, and the higher-frequency variations associated with individual samples (e.g., the high $\mathrm{Al}$-amphiboles at 1630,1760, 1820,1929, and 1980 mbsf, and the low-Al ones at 1600 and $1980 \mathrm{mbsf}$ ).

posed of chlorite where they cut plagioclase and by actinolite where they cut across augite. Chlorite commonly replaces olivine or the rim of olivine phenocrysts whose core is replaced by a mixed-layer chlorite-smectite (Pl. 2).

The chemical compositions of some representative chlorite and mixed-layer chlorite-smectites are given in Table 5. The optical properties of these minerals reflect their chemical composition and structure: chlorite is light green, pleochroic, and shows anomalous brown or purple interference colors or "normal" blue-gray with crossed polars, whereas mixed-layered chlorite-smectite is pleochroic darker green or brownish to yellowish green, with higher birefringence. Analyses plot in the pycnochlorite and diabantite fields of Hey's (1954) classification (Fig. 7). Most of the analyses plot in the fields of Legs 83 and 111 chlorites, but some are slightly enriched in iron.

When plotted on a $\left(\mathrm{Si}+\mathrm{Al}^{\mathrm{tot}}+\mathrm{Fe}+\mathrm{Mg}\right)$ vs. $\left(\mathrm{Al}^{\text {tot }}\right)$ diagram (Schiffman and Fridleifsson, 1991), the Legs 137/140 data plot along the saponite-chlorite trend although most of the points are concentrated around the chlorite end-member.

The percentage $(X)$ of pure chlorite in the mixed-layer chloritesmectite was calculated using Wise's method described in Bettison and Schiffman (1988) and Bevins et al. (1991). Structural formulas and corresponding $X$ values are presented in Table 5. Chlorite-smectite in veins is more chloritic than that replacing olivine in the host-rock of the same sample. Chlorite-smectite in the rim of olivine is more chloritic than that replacing the core. Chlorite replacing clinopyroxene is not very common or abundant. It contains significant amounts of $\mathrm{Cr}_{2} \mathrm{O}_{3}$ (up to $2.7 \mathrm{wt} \%$ ), reflecting the influence of the precursor mineral composition.

Variation of chlorite composition with depth (Fig. 8) shows zones of variable iron enrichment. The depth intervals with particularly low $\mathrm{FeO}_{\mathrm{t}}$ concentrations (i.e., 1700-1820, 1905-1938, and 1980-1985 mbsf) correspond to the occurrence of fresh olivine relicts and/or with the replacement of olivine by talc, and are negatively correlated with the calculated proportion of chlorite in phyllosilicates ( $X$ value). The zones where phyllosilicates are $\mathrm{Mg}$-rich and $\mathrm{Fe}$-poor (occurrence of talc and low-Fe chlorite) are thought to have been altered under relatively low water/rock ratios.

Variations of $\mathrm{MnO}$ with depth approximately parallel those of $\mathrm{FeO}$, with a clear MnO enrichment between 1820 and $1910 \mathrm{~m}$.

Considering the entire dike section (Fig. 8), the iron content of chlorite and chlorite-smectite is low in the stockwork zone, increases with depth down to $1560 \mathrm{mbsf}$ (end of Leg 111), and generally decreases again in the deepest 400 meters, with the local low values mentioned above.

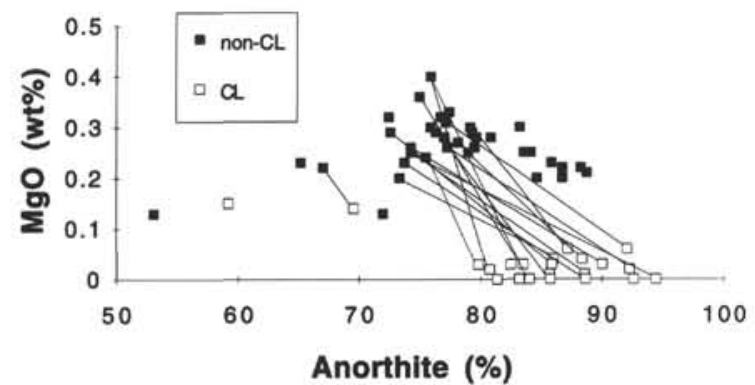

Figure 6. Results of electron microprobe analysis of feldspars from alteration patches or vein halos. In each case, the cathodoluminescent character of the analyzed spot was determined, because primary unaltered igneous plagioclase cores are nonluminescent, whereas secondary, inclusion-filled replacement rims luminesce brightly. The primary igneous plagioclase (closed symbols) is typically bytownite $\left(\mathrm{An}_{70-90}\right)$ and contains $0.1 \%-0.5 \mathrm{wt} \% \mathrm{MgO}$. The secondary plagioclase rims (open symbols) are more calcic bytownite and anorthite, and contain $<0.1 \mathrm{wt} \% \mathrm{MgO}$. Tie lines connect analyses taken within several tens of microns across a core-rim boundary. A similar plot for $\mathrm{FeO}$ shows a similar situation, although the $\mathrm{FeO}$ concentrations of the two groups overlap somewhat. $\mathrm{CL}$ refers to luminescent rims of plagioclase exhibiting cathodoluminescence.

\section{Talc}

Talc is easily distinguished from other phyllosilicates in thin section because it is colorless and has much higher birefringence. It is common from 1705 to $1745 \mathrm{mbsf}, 1760$ to $1790 \mathrm{mbsf}, 1815$ to 1825 mbsf, 1910 to $1925 \mathrm{mbsf}$, and $1992 \mathrm{mbsf}$ as a total or partial replacement of olivine, and is associated with magnetite and/or hematite. Olivine relics occur only in the talc-bearing samples.

Trace $\mathrm{Al}$ and interlayer cations plus minor $\mathrm{Fe}$ in Legs 137/140 talc (Table 6) suggest the presence of chlorite or mixed-layered chloritesmectite in the talc, as in shallower dikes (Alt et al., 1986a, 1989b). The $\mathrm{Fe} / \mathrm{Al}$ ratio of talc (4.5) is much greater than that for chlorite $(0.9)$ or smectite (1.5) in Legs 137/140 rocks, however, suggesting that the talc is Fe-bearing. Attributing all the $\mathrm{Al}$ in the talc analyses to the presence of chlorite indicates that about $75 \%$ of the $\mathrm{Fe}$ in the analyses is contained in the talc. This is confirmed by HRTEM observation of chlorite layers in talc: pure talc layers have been analyzed by HRTEM and contain $2 \mathrm{wt} \% \mathrm{FeO}$.

Talc is interpreted as reflecting a low water/rock ratio alteration process, which can occur at temperatures from $250^{\circ} \mathrm{C}$ to $500^{\circ}-600^{\circ} \mathrm{C}$ (Shipboard Scientific Party, 1992b).

\section{Serpentine}

Serpentine was tentatively identified optically during Leg 140 as a partial replacement of olivine, typically forming a band located between a talc rim and the fresh olivine core. No serpentine was found during subsequent onshore microprobe studies, but lizardite and chrysotile replacing olivine were observed with HRTEM.

\section{Epidote}

Epidote occurs sporadically in veins, vugs, and as small patches replacing plagioclase. Epidote does not coexist with relict fresh olivine, and has been found in only two talc-bearing samples. Epidote generally forms in veins from evolved fluids, so the lack of epidote in the rocks containing relict olivine and talc may be attributed to the low permeability of the crust where these rocks occur. Epidote commonly coexists with anhydrite, except in the deeper $80 \mathrm{~m}$, where epidote occurs without anhydrite.

The range of composition of epidote from Legs 137/140 (Table 7) is the same as in Leg 83 (Alt et al., 1986a; Laverne, 1987b) and Leg 
Table 2. Representative amphibole analyses, Hole 504B, Legs 137/140.

\begin{tabular}{|c|c|c|c|c|c|c|c|c|c|c|c|c|c|}
\hline $\begin{array}{l}\text { Core, section, } \\
\text { interval }(\mathrm{cm})\end{array}$ & $\begin{array}{c}\text { Expandable } \\
\text { depth }\end{array}$ & Analysis & Occurrence & Mineral & $\mathrm{SiO}_{2}$ & $\mathrm{Al}_{2} \mathrm{O}_{3}$ & $\mathrm{FeO}_{\mathrm{t}}$ & $\mathrm{MnO}$ & $\mathrm{MgO}$ & $\mathrm{CaO}$ & $\mathrm{Na}_{2} \mathrm{O}$ & $\mathrm{K}_{2} \mathrm{O}$ & $\mathrm{TiO}_{2}$ \\
\hline $173 \mathrm{R}-1,8-10$ & 1570.4 & AA17 & ground. & Cumm & 50.39 & 1.78 & 22.15 & 0.67 & 13.43 & 6.76 & 0.25 & 0.01 & 0.14 \\
\hline $173 R-1,77-79$ & 1573.1 & B2184 & interst/.cpx & $\mathrm{Fe}$-Act & 52.02 & 2.20 & 20.90 & 0.44 & 10.12 & 12.35 & 0.17 & 0.03 & 0.06 \\
\hline $176 \mathrm{R}-1,15-17$ & 1596.4 & $\mathrm{Al}$ & vein & Act & 52.70 & 1.27 & 20.87 & 0.38 & 11.61 & 10.71 & 0.09 & 0.01 & 0.06 \\
\hline & 1596.4 & A17 & vein & Fe-Act & 52.60 & 1.23 & 21.11 & 0.34 & 11.26 & 10.72 & 0.06 & 0.01 & 0.07 \\
\hline & $\begin{array}{l}1596.4 \\
15964\end{array}$ & A9 & vein & $\mathrm{Mg}$-Hbl & $\begin{array}{l}46.06 \\
51.88\end{array}$ & $\begin{array}{ll}11.00 \\
3,37\end{array}$ & 18.46 & 0.31 & 12.14 & $\begin{array}{l}8.23 \\
0.78\end{array}$ & 1.54 & 0.00 & 0.15 \\
\hline $177 \mathrm{R}-1,11-15$ & $\begin{array}{l}1596.4 \\
1606.2\end{array}$ & $\begin{array}{l}\text { A233 } \\
\text { B2108 }\end{array}$ & $\begin{array}{l}\text { halo } \\
\text { vein }\end{array}$ & Act & $\begin{array}{l}51.88 \\
52.51\end{array}$ & $\begin{array}{l}3.37 \\
2.56\end{array}$ & $\begin{array}{l}16.76 \\
17.91\end{array}$ & $\begin{array}{l}0.35 \\
0.27\end{array}$ & $\begin{array}{l}14.59 \\
13.59\end{array}$ & $\begin{array}{r}9.78 \\
10.61\end{array}$ & $\begin{array}{l}0.29 \\
0.30\end{array}$ & $\begin{array}{l}0.00 \\
0.00\end{array}$ & $\begin{array}{l}0.47 \\
0.24\end{array}$ \\
\hline $186 \mathrm{R}-1,21-23$ & 1627.1 & $\begin{array}{l}827090 \\
V\end{array}$ & $\begin{array}{l}\text { vein } \\
\text { vein }\end{array}$ & $\begin{array}{l}\text { Act } \\
\text { Act }\end{array}$ & $\begin{array}{l}32.51 \\
52.21\end{array}$ & 2.41 & $\begin{array}{l}1.91 \\
19.65\end{array}$ & $\begin{array}{l}0.27 \\
0.43\end{array}$ & $\begin{array}{l}13.39 \\
11.47\end{array}$ & 11.64 & 0.31 & $\begin{array}{l}0.00 \\
0.00\end{array}$ & $\begin{array}{l}0.24 \\
0.11\end{array}$ \\
\hline & 1627.1 & $\mathrm{~V} 4095$ & vein wall & Act & 52.45 & 2.58 & 16.94 & 0.29 & 14.22 & 11.02 & 0.31 & 0.00 & 0.23 \\
\hline $186 \mathrm{R}-1,57-60$ & 1628.1 & $\mathrm{~V} 4100$ & vein & $\mathrm{Act}-\mathrm{Hbl}$ & 50.71 & 5.57 & 14.95 & 0.28 & 14.26 & 11.87 & 0.90 & 0.02 & 0.20 \\
\hline & 1628.1 & V4102 & vein & $\mathrm{Mg}-\mathrm{Hbl}$ & 48.01 & 6.73 & 14.77 & 0.25 & 12.76 & 11.67 & 0.82 & 0.02 & 0.27 \\
\hline & 1628.1 & V4107 & vein & Act & 51.98 & 1.91 & 20.61 & 0.34 & 11.00 & 10.89 & 0.26 & 0.00 & 0.07 \\
\hline $186 \mathrm{R}-1,140-143$ & 1630.1 & B3107 & vein & Act & 52.74 & & 13.14 & 0.18 & 16.11 & 10.61 & 0.50 & 0.00 & 0.54 \\
\hline $186 \mathrm{R}-2,25-28$ & 1631.4 & A29 & halo & Act-Hbl & 51.86 & 3.27 & 13.01 & 0.27 & 16.27 & 10.97 & 0.49 & 0.00 & 0.35 \\
\hline & 1631.4 & A32 & vein & $\mathrm{Act}-\mathrm{Hbl}$ & 51.40 & 4.11 & 13.58 & 0.34 & 15.30 & 11.43 & 0.57 & 0.02 & 0.78 \\
\hline & 1631.4 & A33 & vein & $\mathrm{Mg}-\mathrm{Hbl}$ & 48.85 & 6.46 & 15.07 & 0.23 & 13.23 & 11.38 & 1.14 & 0.02 & 0.39 \\
\hline 189R-1, $105-106$ & 1652.9 & B2001 & yein & Act & 53.15 & 1.93 & 15.23 & 0.19 & 14.52 & 11.41 & 0.48 & 0.01 & 0.35 \\
\hline & 1652.9 & B2014 & halo & $\mathrm{Fe}$-Act & 51.44 & 2.47 & 20.49 & 0.50 & 10.79 & 11.04 & 0.28 & 0.01 & 0.31 \\
\hline & $\begin{array}{l}1652.9 \\
16530\end{array}$ & B2015 & halo & Cumm & 49.66 & 3.30 & 25.87 & 0.70 & 9.53 & 8.10 & 0.44 & 0.05 & 0.39 \\
\hline $189 \mathrm{R}-1,106-110$ & $\begin{array}{l}1653.0 \\
1653.0\end{array}$ & $\begin{array}{l}\text { V5131 } \\
\text { V5132 }\end{array}$ & $\begin{array}{l}\text { vein } \\
\text { vein }\end{array}$ & $\mathrm{Act}$ & $\begin{array}{l}52.96 \\
51.72\end{array}$ & $\begin{array}{l}2.06 \\
3.74\end{array}$ & 16.25 & 0.29 & $\begin{array}{l}14.17 \\
1306\end{array}$ & $\begin{array}{l}11.19 \\
1082\end{array}$ & 0.32 & 0.00 & 0.32 \\
\hline & $\begin{array}{l}16533.0 \\
1653.0\end{array}$ & $\begin{array}{l}\text { V5132 } \\
\text { V5144 }\end{array}$ & $\begin{array}{l}\text { vein } \\
\text { vein }\end{array}$ & Act & $\begin{array}{l}51.72 \\
51.59\end{array}$ & 3,74 & $\begin{array}{l}16.10 \\
26.81\end{array}$ & 0.34 & $\begin{array}{l}13.96 \\
7.89\end{array}$ & 10.82 & 0.49 & 0.02 & 0.28 \\
\hline $189 \mathrm{R}-2,76-79$ & $\begin{array}{l}1635.0 \\
1654.3\end{array}$ & $\begin{array}{l}\text { B2157 } \\
\text { B2157 }\end{array}$ & 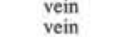 & $\begin{array}{l}\text { Fe-Act } \\
\text { Act }\end{array}$ & $\begin{array}{l}51.59 \\
51.22\end{array}$ & $\begin{array}{l}1.28 \\
2.41\end{array}$ & $\begin{array}{l}26.81 \\
18.74\end{array}$ & $\begin{array}{l}0.61 \\
0.12\end{array}$ & $\begin{array}{r}7.89 \\
11.35\end{array}$ & $\begin{array}{l}10.12 \\
11.43\end{array}$ & $\begin{array}{l}0.21 \\
0.50\end{array}$ & $\begin{array}{l}0.03 \\
0.13\end{array}$ & $\begin{array}{l}0.12 \\
0.21\end{array}$ \\
\hline & 1654.3 & B2173 & ground. & $\mathrm{Fe}-\mathrm{Act}$ & 50.73 & 1.76 & 26.63 & 0.46 & 7.08 & 11.26 & 0.29 & $\begin{array}{l}0.13 \\
0.05\end{array}$ & $\begin{array}{l}0.21 \\
0.09\end{array}$ \\
\hline $189 \mathrm{R}-2,100-104$ & 1655.0 & A45 & patch & Act-Hbl & 50.54 & 3.98 & 16.01 & 0.22 & 12.73 & 11.80 & 0.30 & 0.01 & 0.47 \\
\hline 191R-1, 145-148 & 1670.5 & B3060 & ground. & $\mathrm{Fe}$-Act & 48.33 & 3.87 & 26.18 & 0.35 & 7.92 & 8.67 & 0.69 & 0.04 & 0.39 \\
\hline & $\begin{array}{l}1670.5 \\
16846\end{array}$ & B3061 & ground. & $\mathrm{Fe}-\mathrm{Act}-\mathrm{Hbl}$ & 47.84 & 3.95 & 25.73 & 0.22 & 7.65 & 9.71 & 0.81 & 0.16 & 0.43 \\
\hline $194 R-1,59-61$ & 1684.6 & A56 & patch & Act-Hbl & 50.77 & 0.54 & 14.79 & 0.47 & 12.12 & 19.22 & 0.29 & 0.00 & 0.16 \\
\hline & 1684.6 & A58 & patch & Act & 51.09 & 2.58 & 18.89 & 0.31 & 11.46 & 11.82 & 0.25 & 0.02 & 0.08 \\
\hline $196 \mathrm{R}-1,29-33$ & 1700.5 & B3079 & vein & Act & 51.83 & 3.38 & 18.04 & 0.45 & 12.48 & 10.72 & 0.36 & 0.05 & 0.24 \\
\hline $197 \mathrm{R}-1,44-46$ & 1704.9 & B3070 & ground. & Act-Hbl & 51.29 & 4.49 & 17.28 & 0.33 & 13.27 & 10.72 & 0.57 & 0.07 & 0.20 \\
\hline $197 \mathrm{R}-1,100-102$ & 1708.3 & A57 & $\begin{array}{l}\text { vein wall } \\
\text { ver }\end{array}$ & Act-Hbl & 50.81 & 4.39 & 14.88 & 0.40 & 13.25 & 11.17 & 0.71 & 0.03 & 0.34 \\
\hline & 1708.3 & A60 & vein & Act & 51.81 & 2.63 & 17.50 & 0.21 & 12.63 & 11.42 & 0.24 & 0.02 & 0.18 \\
\hline 199R-1, $14-16$ & 1720.4 & B2135 & ground. & Act-Hbl & $\begin{array}{l}51.74 \\
5100\end{array}$ & 3.59 & $\begin{array}{l}15.92 \\
1053\end{array}$ & 0.27 & 14.65 & 11.93 & 0.75 & 0.09 & 0.04 \\
\hline $200 R-2,18-24$ & $\begin{array}{l}1731.7 \\
1731.7\end{array}$ & $\begin{array}{l}\text { V } 33058 \\
\text { V 3062 }\end{array}$ & $\begin{array}{c}\text { ground. } \\
\text { yein }\end{array}$ & Act & $\begin{array}{r}51.09 \\
4982\end{array}$ & 3.11 & $\begin{array}{l}19.53 \\
16.99\end{array}$ & 0.55 & $\begin{array}{l}13.22 \\
13.37\end{array}$ & $\begin{array}{r}9.00 \\
1066\end{array}$ & $\begin{array}{l}0.69 \\
0.99\end{array}$ & 0.00 & $\begin{array}{l}0.22 \\
0.09\end{array}$ \\
\hline & $\begin{array}{l}1731.7 \\
1731.7\end{array}$ & $\begin{array}{l}\text { V3062 } \\
\text { V3064 }\end{array}$ & $\begin{array}{l}\text { vein } \\
\text { vein }\end{array}$ & $\begin{array}{l}\text { Act-Hbl } \\
\text { Cumm }\end{array}$ & $\begin{array}{l}49.82 \\
53.30\end{array}$ & $\begin{array}{l}5.31 \\
1.97\end{array}$ & $\begin{array}{l}16.99 \\
19.02\end{array}$ & $\begin{array}{l}0.47 \\
0.75\end{array}$ & $\begin{array}{l}13.37 \\
17.12\end{array}$ & $\begin{array}{r}10.66 \\
4.16\end{array}$ & $\begin{array}{l}0.99 \\
0.46\end{array}$ & $\begin{array}{l}0.01 \\
0.02\end{array}$ & $\begin{array}{l}0.09 \\
0.20\end{array}$ \\
\hline & $\begin{array}{l}1731.1 \\
1731.7\end{array}$ & v3066 & $\begin{array}{l}\text { vein } \\
\text { vein }\end{array}$ & $\begin{array}{l}\text { Cumm } \\
\text { Cumm }\end{array}$ & $\begin{array}{l}53.30 \\
52.23\end{array}$ & $\begin{array}{l}1.97 \\
3.25\end{array}$ & $\begin{array}{l}19.02 \\
16.92\end{array}$ & $\begin{array}{l}0.75 \\
0.53\end{array}$ & $\begin{array}{l}17.12 \\
15.56\end{array}$ & $\begin{array}{l}4.16 \\
7.11\end{array}$ & $\begin{array}{l}0.46 \\
0.68\end{array}$ & $\begin{array}{l}0.02 \\
0.01\end{array}$ & $\begin{array}{l}0.20 \\
0.10\end{array}$ \\
\hline 200R-2, 101-104 & 1733.4 & B2101 & vein & Act & 52.32 & 3.30 & $\begin{array}{l}16.92 \\
16.60\end{array}$ & $\begin{array}{l}0.53 \\
0.51\end{array}$ & $\begin{array}{l}15.56 \\
14.56\end{array}$ & 9.48 & $\begin{array}{l}0.08 \\
0.57\end{array}$ & $\begin{array}{l}0.01 \\
0.02\end{array}$ & $\begin{array}{l}0.10 \\
0.21\end{array}$ \\
\hline 200R-3, 108-112 & 1736.0 & V6176 & vein & Act & 51.06 & 1.37 & 14.63 & 0.68 & 12.26 & 12.93 & 0.32 & 0.02 & 0.20 \\
\hline $201 R-1,0-4$ & 1739.3 & B3132 & ground. & Mg-Hbl & 50.29 & 5.87 & 13.27 & 0.25 & 14.63 & 11.97 & 0.83 & 0.01 & 0.38 \\
\hline $203 \mathrm{R}-1,9-10$ & 1750.9 & B3021 & vein & Act-Hbl & 52.27 & 4.63 & 14.25 & 0.19 & 14.90 & 11.73 & 0.59 & 0.02 & 0.44 \\
\hline $203 R-1,33-36$ & 1753.9 & V2045 & ground. & Act-Hbl & 50.25 & 5.03 & 13.94 & 0.18 & 14.15 & 10.29 & 0.93 & 0.06 & 0.59 \\
\hline & 1753.9 & V2033 & vein & Act & 53.29 & 2.66 & 16.41 & 0.33 & 14.66 & 10.34 & 0.39 & 0.02 & 0.31 \\
\hline & 1753.9 & V2037 & vein & $\mathrm{Act}-\mathrm{Hbl}$ & 49.12 & 4.64 & 16.38 & 0.33 & 12.74 & 9.61 & 0.87 & 0.02 & 0.27 \\
\hline & 1753.9 & V2039 & shear zone & Act & 52.73 & 3.11 & 15.57 & 0.23 & 14.34 & 11.21 & 0.43 & 0.00 & 0.27 \\
\hline $204 \mathrm{R}-1,11-14$ & 1756.6 & V5165 & vein & Act-Hbl & 51.07 & 3.00 & 17.65 & 0.42 & 14.56 & 9.78 & 0.64 & 0.00 & 0.28 \\
\hline & $\begin{array}{l}1756.6 \\
17566\end{array}$ & $\begin{array}{l}\text { V5166 } \\
\text { V5169 }\end{array}$ & vein & Act & 53.28 & 1.68 & $\begin{array}{l}15.34 \\
1455\end{array}$ & 0.36 & 15.98 & 10.52 & 0.27 & 0.02 & 0.16 \\
\hline 205R-1, 13-16 & $\begin{array}{l}17566.6 \\
1757.4\end{array}$ & $\begin{array}{l}\text { V169 } \\
\text { A62 }\end{array}$ & $\begin{array}{l}\text { ground. } \\
\text { vein }\end{array}$ & $\begin{array}{l}\mathrm{Act}-\mathrm{Hbl} \\
\mathrm{Mg}-\mathrm{Hbl}\end{array}$ & $\begin{array}{l}50.49 \\
47.69\end{array}$ & $\begin{array}{l}3.35 \\
7.52\end{array}$ & $\begin{array}{l}14.55 \\
14.96\end{array}$ & 0.22 & $\begin{array}{l}14.05 \\
1.74\end{array}$ & 12.22 & $\begin{array}{l}0.40 \\
1.54\end{array}$ & $\begin{array}{l}0.00 \\
0.12\end{array}$ & 0.52 \\
\hline & 1757.4 & $\begin{array}{l}\mathrm{A} 62 \\
\mathrm{~A} 70\end{array}$ & $\begin{array}{l}\text { vein } \\
\text { halo }\end{array}$ & $\begin{array}{l}\mathrm{Mg}-\mathrm{H} \text { - } \\
\mathrm{Mg} \mathrm{Hb}\end{array}$ & $\begin{array}{l}4.69 \\
50.72\end{array}$ & $\begin{array}{l}7.52 \\
4.81\end{array}$ & $\begin{array}{l}14.96 \\
1387\end{array}$ & 0.22 & $\begin{array}{l}14.74 \\
14.01\end{array}$ & $\begin{array}{l}11.05 \\
11.59\end{array}$ & $\begin{array}{l}1.54 \\
0.64\end{array}$ & $\begin{array}{l}0.12 \\
0.03\end{array}$ & $\begin{array}{l}0.64 \\
0.50\end{array}$ \\
\hline 208R-1, 88-91 & 1776.2 & A70 & $\begin{array}{l}\text { halo } \\
\text { hal }\end{array}$ & $\mathrm{Mg}-\mathrm{Hbl}$ & 48.02 & 4.45 & $\begin{array}{l}13.87 \\
19.50\end{array}$ & $\begin{array}{l}0.31 \\
0.36\end{array}$ & $\begin{array}{l}14.01 \\
12.01\end{array}$ & $\begin{array}{l}11.59 \\
10.43\end{array}$ & $\begin{array}{l}0.04 \\
0.52\end{array}$ & $\begin{array}{l}0.03 \\
0.02\end{array}$ & $\begin{array}{l}0.90 \\
0.19\end{array}$ \\
\hline $208 \mathrm{R}-3,1-4$ & 1787.5 & A73 & halo & $\mathrm{Act}-\mathrm{Hbl}$ & 50.72 & 4.81 & 13.87 & 0.31 & 14.01 & 11.59 & 0.64 & 0.03 & $\begin{array}{l}0.19 \\
0.50\end{array}$ \\
\hline & 1787.5 & A75 & halo & $\mathrm{Mg}-\mathrm{Hbl}$ & 49.55 & 12.40 & 8.27 & 0.17 & 8.99 & 16.59 & 0.33 & 0.00 & 0.2 \\
\hline 209R-2, 146-149 & 1794.7 & V6182 & vein & Act-Hbl & 50.80 & 4.05 & 15.65 & 0.27 & 13.22 & 11.2 & 0. & 0.03 & $0.2>-2$ \\
\hline & 1794.7 & V6183 & plag. crack & Act & 51.16 & 4.06 & 16.11 & 0.26 & 13.16 & 10.7 & 0. & 0.02 & $0.2-2>$ \\
\hline $210 R-1,56-60$ & 1795.9 & B3116 & vein & Act & 53.11 & 2.40 & 17.78 & 0.11 & 12.04 & 12.2 & 0.31 & 0.01 & 0.16 \\
\hline $213 \mathrm{R}-1$ & 1812.8 & V1025 & vein & $\mathrm{Mg}-\mathrm{Hbl}$ & 49.05 & 5.86 & 14.94 & 0.18 & 14.04 & 12.19 & 1.31 & 0.11 & 1.94 \\
\hline & 1812.8 & v1027 & vein & Act & 52.88 & 2.04 & 18.21 & 0.44 & 12.09 & 11.45 & 0.25 & 0.00 & 0.11 \\
\hline & 1812.8 & V1028 & ground. & Act-Hbl & 51.78 & 3.98 & 16.74 & 0.38 & 13.02 & 11.16 & 0.57 & 0.01 & 0.21 \\
\hline $213 R-1,45-47$ & 1814.9 & P67 & patch & Act-Hbl & 50.07 & 4.72 & 11.69 & 0.13 & 15.33 & 11.52 & 0.64 & 0.02 & 0.16 \\
\hline $214 R-1,28-30$ & $\begin{array}{l}1819.0 \\
18100\end{array}$ & B2140 & patch & Act-Hbl & 50.75 & 5.94 & 15.66 & 0.26 & $\begin{array}{l}13.35 \\
1506\end{array}$ & 11.90 & 0.89 & 0.02 & 0.40 \\
\hline & $\begin{array}{l}1819.0 \\
18190\end{array}$ & $\begin{array}{l}\text { B2149 } \\
B\end{array}$ & vein & Edenite & 47.01 & $\begin{array}{r}6.39 \\
5.06\end{array}$ & 12.74 & 0.07 & $\begin{array}{l}15.96 \\
1544\end{array}$ & 10.33 & 1.74 & 0.07 & $\begin{array}{l}1.30 \\
068\end{array}$ \\
\hline & $\begin{array}{l}\begin{array}{l}1819.0 \\
1819.0\end{array}\end{array}$ & $\begin{array}{l}\text { B2150 } \\
\text { B2153 }\end{array}$ & $\begin{array}{l}\text { vein } \\
\text { vein }\end{array}$ & $\begin{array}{l}\mathrm{Mg}-\mathrm{Hbl} \\
\mathrm{Act}-\mathrm{bb}\end{array}$ & $\begin{array}{l}51.18 \\
50.88\end{array}$ & 5.06 & $\begin{array}{l}13.67 \\
14.89\end{array}$ & $\begin{array}{l}0.12 \\
0.26\end{array}$ & $\begin{array}{r}15.44 \\
114.14\end{array}$ & $\begin{array}{l}11.10 \\
11.55\end{array}$ & 1.19 & $\begin{array}{l}0.16 \\
0.04\end{array}$ & $\begin{array}{l}0.68 \\
0.52\end{array}$ \\
\hline $214 R-1,67-70$ & $\begin{array}{l}1819.5 \\
1819.5\end{array}$ & B2187 & $\begin{array}{l}\text { vein } \\
\text { vein }\end{array}$ & $\begin{array}{l}\text { Act-Hbl } \\
\mathrm{Mg}-\mathrm{Hbl}\end{array}$ & $\begin{array}{l}50.88 \\
48.02\end{array}$ & $\begin{array}{l}5.56 \\
6.91\end{array}$ & $\begin{array}{l}14.89 \\
13.87\end{array}$ & 0.26 & $\begin{array}{l}14.14 \\
14.70\end{array}$ & 11.05 & $\begin{array}{l}0.81 \\
1.23\end{array}$ & $\begin{array}{l}0.04 \\
0.06\end{array}$ & 1.02 \\
\hline & 1819.5 & B2198 & vein & Edenite & 48.83 & $\begin{array}{l}6.91 \\
6.68\end{array}$ & $\begin{array}{l}14.81 \\
14.08\end{array}$ & $\begin{array}{l}0.21 \\
0.32\end{array}$ & $\begin{array}{l}15.46 \\
15.46\end{array}$ & 10.87 & 2.02 & $\begin{array}{l}0.00 \\
0.06\end{array}$ & $\begin{array}{l}1.02 \\
0.09\end{array}$ \\
\hline $214 \mathrm{R}-1,76-78$ & 1819.6 & P2 & ground. & Act-Hbl & 50.44 & 3.68 & 13.09 & 0.18 & 16.11 & 10.54 & 0.74 & 0.04 & 0.44 \\
\hline & $\begin{array}{l}1819.6 \\
18196\end{array}$ & P15 & ground. & Edenite & 47.16 & 7.8 & 13.12 & 0.15 & 15. & 10.51 & 2. & 0.05 & 0. \\
\hline $214 \mathrm{R}-2=5$ & $\begin{array}{l}1819.6 \\
18209\end{array}$ & $\begin{array}{c}\text { P17 } \\
\text { B3123 }\end{array}$ & $\begin{array}{c}\text { ground. } \\
\text { yein }\end{array}$ & Act & $\begin{array}{l}52.07 \\
53.59\end{array}$ & $\begin{array}{r}2.00 \\
1.48\end{array}$ & $\begin{array}{l}17.66 \\
18.44\end{array}$ & $\begin{array}{l}0.39 \\
0.30\end{array}$ & 14.67 & $\begin{array}{r}9.48 \\
1130\end{array}$ & 0.54 & 0.06 & $\begin{array}{l}0.36 \\
0.25\end{array}$ \\
\hline $214 R-2,0-5$ & $\begin{array}{l}1820.9 \\
18209\end{array}$ & $\begin{array}{l}\text { B3123 } \\
\text { B3126 }\end{array}$ & $\begin{array}{l}\text { vein } \\
\text { vein }\end{array}$ & $\begin{array}{l}\text { Act } \\
\text { Act }\end{array}$ & $\begin{array}{l}53.59 \\
53.64\end{array}$ & $\begin{array}{l}1.48 \\
2.35\end{array}$ & $\begin{array}{r}18.44 \\
15.73\end{array}$ & 0.30 & $\begin{array}{l}13.21 \\
1.44\end{array}$ & $\begin{array}{l}11.39 \\
10.81\end{array}$ & $\begin{array}{l}0.36 \\
0.50\end{array}$ & $\begin{array}{l}0.02 \\
0.00\end{array}$ & $\begin{array}{l}0.25 \\
0.32\end{array}$ \\
\hline $214 \mathrm{R}-2,29-32$ & $\begin{array}{l}1820.9 \\
1821.4\end{array}$ & $\begin{array}{l}\text { B3126 } \\
\text { P24 }\end{array}$ & $\begin{array}{l}\text { veen } \\
\text { ground. }\end{array}$ & $\begin{array}{c}\mathrm{Act} \\
\mathrm{Mg}-\mathrm{Hbl}\end{array}$ & $\begin{array}{l}53.64 \\
46.55\end{array}$ & $\begin{array}{l}2.35 \\
5.87\end{array}$ & $\begin{array}{l}15.73 \\
19.78\end{array}$ & $\begin{array}{l}0.16 \\
0.18\end{array}$ & $\begin{array}{l}\begin{array}{l}14.44 \\
11.30\end{array}\end{array}$ & $\begin{array}{l}10.81 \\
10.02\end{array}$ & $\begin{array}{l}0.50 \\
1.10\end{array}$ & $\begin{array}{l}0.00 \\
0.06\end{array}$ & $\begin{array}{l}0.32 \\
0.15\end{array}$ \\
\hline $223 \mathrm{R}-1,9-11$ & 189 & B3023 & vein & Act & 51.66 & 3.82 & 15.27 & 0.19 & 13.78 & 12.08 & 0. & 0.04 & 0.1 \\
\hline & & $\begin{array}{l}B 3025 \\
B 3020\end{array}$ & halo & Act-Hbl & 50.12 & 4. & 15.69 & 0.25 & 13.31 & 11.45 & 0.8 & 0.01 & 0.68 \\
\hline & 1896.8 & B3030 & ground. & $\mathrm{Mg}-\mathrm{Hbl}$ & 49.05 & 6.9 & 15.94 & 0.40 & 13.86 & 10.19 & 0.7 & 0.01 & 0. \\
\hline $225 R-1,91-93$ & $\begin{array}{l}1915.2 \\
1915.2\end{array}$ & A96 & halo & Act-Hbl & 51.05 & $\begin{array}{l}4.78 \\
5.72\end{array}$ & 14.02 & 0.38 & 15.00 & 9.97 & 0.77 & 0.02 & 0.25 \\
\hline $225 R-2,68-70$ & $\begin{array}{l}1915.2 \\
1919.5\end{array}$ & $\begin{array}{l}\text { A100 } \\
\text { AA127 }\end{array}$ & $\begin{array}{l}\text { vein } \\
\text { vein }\end{array}$ & $\begin{array}{l}\mathrm{Mg}-\mathrm{Hb} \\
\mathrm{Ac}-\mathrm{bb}\end{array}$ & $\begin{array}{l}49.20 \\
49.98\end{array}$ & $\begin{array}{l}5.73 \\
2.94\end{array}$ & $\begin{array}{l}14.15 \\
15.22\end{array}$ & 0.07 & $\begin{array}{l}13.54 \\
14.59\end{array}$ & $\begin{array}{l}11.48 \\
11.80\end{array}$ & $\begin{array}{l}0.96 \\
0.38\end{array}$ & 0.07 & 0.47 \\
\hline & $\begin{array}{l}1919 \\
1919\end{array}$ & $\begin{array}{l}\text { AA127 } \\
\text { AA129 }\end{array}$ & $\begin{array}{l}\text { halo } \\
\text { halo }\end{array}$ & $\begin{array}{l}\text { Act-Hbl } \\
\mathrm{Mg}-\mathrm{Hbl}\end{array}$ & $\begin{array}{l}49.48 \\
45.41\end{array}$ & 7.40 & $\begin{array}{l}15.22 \\
14.78\end{array}$ & $\begin{array}{l}0.20 \\
0.19\end{array}$ & $\begin{array}{l}14.59 \\
13.39\end{array}$ & $\begin{array}{l}11.80 \\
11.94\end{array}$ & 1.2 & 0.05 & 0.4 \\
\hline & 191 & AAl & host & $\mathrm{Fe}-\mathrm{Hbl}$ & 45.76 & 3.71 & 26.34 & 0.28 & 7. & 9.55 & 0. & 0. & 0. \\
\hline $226 \mathrm{R}-1,99-102$ & 1921.4 & B207 & ground. & Act-Hbl & 50.46 & 4.57 & 15.67 & 0.36 & 14. & 11.90 & 0. & 0. & \\
\hline & 1921.4 & B2074 & ground. & Act & 52.25 & 3.51 & 17.25 & 0.36 & 14.16 & 10.61 & 0.53 & 0.06 & 0.1 \\
\hline $226 \mathrm{R}-3,5-9$ & $\begin{array}{l}1924.0 \\
19240\end{array}$ & $\begin{array}{l}\text { B2083 } \\
\text { B } 2084\end{array}$ & $\begin{array}{l}\text { ground. } \\
\text { ground }\end{array}$ & Act & $\begin{array}{l}51.68 \\
5506\end{array}$ & $\begin{array}{l}2.75 \\
507\end{array}$ & $\begin{array}{l}19.50 \\
1552\end{array}$ & 0.56 & $\begin{array}{l}13.50 \\
13.81\end{array}$ & 8.86 & $\begin{array}{l}0.60 \\
0.76\end{array}$ & & 0.41 \\
\hline & $\begin{array}{l}1924.0 \\
1924.0\end{array}$ & 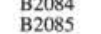 & $\begin{array}{l}\text { ground. } \\
\text { ground. }\end{array}$ & $\mathrm{Act}-\mathrm{Hbl}$ & 50.06 & $\begin{array}{l}5.07 \\
680\end{array}$ & $\begin{array}{l}15.52 \\
1672\end{array}$ & $\begin{array}{l}0.20 \\
0.38\end{array}$ & $\begin{array}{l}13.81 \\
13.65\end{array}$ & $\begin{array}{r}11.36 \\
9.94\end{array}$ & $\begin{array}{l}0.76 \\
1.70\end{array}$ & $\begin{array}{l}0.00 \\
0.03\end{array}$ & $\begin{array}{l}0.30 \\
0.33\end{array}$ \\
\hline $230 \mathrm{R}-1,14-17$ & $\begin{array}{l}1924.0 \\
1953.5\end{array}$ & 5 & $\begin{array}{l}\text { ground, } \\
\text { vein }\end{array}$ & $\begin{array}{c}\text { Mg-Het } \\
\text { Act }\end{array}$ & $\begin{array}{l}48.91 \\
52.95\end{array}$ & $\begin{array}{l}6.72 \\
2.72\end{array}$ & $\begin{array}{l}16.72 \\
16.67\end{array}$ & $\begin{array}{l}0.23 \\
0.23\end{array}$ & $\begin{array}{l}13.65 \\
14.13\end{array}$ & $\begin{array}{l}9.94 \\
10.68\end{array}$ & 0.4 & $\begin{array}{l}0.03 \\
0.03\end{array}$ & 0.1 \\
\hline & & & halo & $\mathrm{Mg}-\mathrm{Hbl}$ & 50.10 & 5.18 & 15.81 & 0.16 & 13.69 & 10.96 & 0. & 0. & 1. \\
\hline & & B3038 & halo & Act-Hbl & 50.83 & 4.39 & 15.69 & 0.28 & 14.13 & 10.33 & 0.88 & 0.09 & \\
\hline & 1953.5 & B3040 & ground. & $\mathrm{Mg}$-Hbl & 48.69 & 7.20 & 16.68 & 0.40 & 12.52 & 10.89 & 1.14 & 0.06 & 0.23 \\
\hline $235 \mathrm{R}-1,8-10$ & 1977.4 & AI & vein & Act-Hbl & 51.44 & 4.21 & 15.16 & 0.27 & 13.66 & 11.50 & 0.55 & 0.02 & 0.37 \\
\hline & $\begin{array}{l}1977.4 \\
1977.4\end{array}$ & Al1 & $\begin{array}{l}\text { vein } \\
\text { halo }\end{array}$ & $\begin{array}{c}\mathrm{Act} \\
\mathrm{Mg}-\mathrm{Hbl}\end{array}$ & $\begin{array}{l}50.81 \\
49.37\end{array}$ & $\begin{array}{l}3.54 \\
5.87\end{array}$ & $\begin{array}{l}15.80 \\
15.40\end{array}$ & 0.26 & $\begin{array}{l}12.72 \\
12.77\end{array}$ & $\begin{array}{l}12.01 \\
11.48\end{array}$ & $\begin{array}{l}0.45 \\
0.79\end{array}$ & $\begin{array}{l}0.01 \\
0.04\end{array}$ & $\begin{array}{l}0.28 \\
0.46\end{array}$ \\
\hline
\end{tabular}

111 (Ishizuka, 1989) rocks. Ishizuka (1989) found evidence of two populations of epidote $\mathrm{XFe}^{3+}$ values $\left(=\mathrm{Fe}^{3+} / \mathrm{Al}+\mathrm{Fe}^{3+}\right)$ in Leg 111 rocks: $\mathrm{XFe}^{3+}$ ranges from 0.16 to 2.2 in epidotes coexisting with prehnite and actinolite, whereas $\mathrm{XFe}^{3+}$ ranges from 2.4 to 2.9 in epidotes coexisting with prehnite and laumontite. $\mathrm{XFe}^{3+}$ ranges from 0.16 to 0.32 , with a mode at 0.26 , in epidotes from Legs $137 / 140$ section.

\section{Prehnite}

Prehnite is widespread in Legs 137/140 dolerites, although it is not important volumetrically. It occurs in interstitial areas or small vugs, together with epidote, laumontite, actinolite and chlorite. In most cases, chlorite rims the amygdules and obviously formed prior to 
Table 2 (continued).

\begin{tabular}{|c|c|c|c|c|c|c|c|c|c|c|c|c|c|c|c|}
\hline Total & $\mathrm{Si}$ & $\mathrm{Al}^{\mathrm{IV}}$ & $\mathrm{Al}^{\mathrm{VI}}$ & $\mathrm{Ti}$ & $\mathrm{Cr}$ & $\mathrm{Fe}^{2+}$ & $\mathrm{Fe}^{3+}$ & $\mathrm{Mn}$ & $\mathrm{Mg}$ & $\mathrm{MgM} 4$ & $\mathrm{Ca}$ & $\mathrm{NaM} 4$ & $\mathrm{NaA}$ & K & Total \\
\hline 95.99 & 7.602 & 0.317 & 0.000 & 0.016 & 0.000 & 2.276 & 0.519 & 0.086 & 3.019 & 0.000 & 1.093 & 0.073 & 0.000 & 0.002 & 15.002 \\
\hline 98.57 & 7.739 & 0.261 & 0.125 & 0.007 & 0.000 & 2.533 & 0.068 & 0.055 & 2.213 & 0.031 & 1.969 & 0.000 & 0.049 & 0.006 & 15.077 \\
\hline 97.70 & 7.829 & 0.171 & 0.051 & 0.007 & 0.000 & 2.462 & 0.130 & 0.048 & 2.570 & 0.000 & 1.705 & 0.026 & 0.000 & 0.002 & 15.002 \\
\hline $\begin{array}{l}97.42 \\
0795\end{array}$ & $\begin{array}{r}7.856 \\
6598\end{array}$ & 0.144 & 0.073 & 0.008 & 0.000 & 2.566 & 0.071 & 0.043 & 2.506 & 0.000 & 1.716 & 0.017 & 0.000 & 0.002 & 15.002 \\
\hline $\begin{array}{l}97.95 \\
97.52\end{array}$ & $\begin{array}{r}6.598 \\
7.533\end{array}$ & $\begin{array}{l}1.402 \\
0.462\end{array}$ & 0.452 & 0.016 & 0.000 & 0.866 & 1.345 & 0.038 & $\begin{array}{r}2.592 \\
3.15\end{array}$ & 0.000 & $\begin{array}{l}1.263 \\
5 ? 2\end{array}$ & $\begin{array}{r}0.428 \\
0.082\end{array}$ & 0.000 & 0.000 & 15.000 \\
\hline $\begin{array}{r}97.52 \\
98.02\end{array}$ & $\begin{array}{l}7.5333 \\
7.682\end{array}$ & $\begin{array}{l}0.467 \\
0.318\end{array}$ & $\begin{array}{l}0.110 \\
0.123\end{array}$ & $\begin{array}{l}0.051 \\
0.026\end{array}$ & $\begin{array}{l}0.000 \\
0.000\end{array}$ & $\begin{array}{l}1.699 \\
2.133\end{array}$ & $\begin{array}{l}0.366 \\
0.058\end{array}$ & $\begin{array}{l}0.043 \\
0.033\end{array}$ & $\begin{array}{l}3.157 \\
2662\end{array}$ & $\begin{array}{l}0.000 \\
0.337\end{array}$ & $\begin{array}{l}1.522 \\
1.663\end{array}$ & $\begin{array}{l}0.082 \\
0.000\end{array}$ & $\begin{array}{l}0.000 \\
0.085\end{array}$ & $\begin{array}{l}0.000 \\
0.000\end{array}$ & $\begin{array}{l}15.000 \\
15 .\end{array}$ \\
\hline 98.27 & 7.715 & 0.285 & 0.135 & $\begin{array}{l}0.026 \\
0.012\end{array}$ & $\begin{array}{l}0.000 \\
0.001\end{array}$ & $\begin{array}{l}2.1333 \\
2.393\end{array}$ & $\begin{array}{l}0.058 \\
0.035\end{array}$ & $\begin{array}{l}0.033 \\
0.054\end{array}$ & $\begin{array}{l}2.626 \\
2.639\end{array}$ & $\begin{array}{l}0.337 \\
0.157\end{array}$ & $\begin{array}{l}1.663 \\
1.843\end{array}$ & $\begin{array}{l}0.000 \\
0.000\end{array}$ & $\begin{array}{l}0.085 \\
0.089\end{array}$ & $\begin{array}{l}0.000 \\
0.000\end{array}$ & $\begin{array}{l}15.104 \\
15.100\end{array}$ \\
\hline 98.72 & 7.598 & 0.402 & 0.038 & 0.025 & 0.069 & 1.894 & 0.158 & 0.036 & 2.780 & 0.290 & 1.710 & 0.000 & 0.087 & 0.000 & $\begin{array}{l}15.130 \\
15\end{array}$ \\
\hline 98.87 & 7.304 & 0.696 & 0.250 & 0.022 & 0.000 & 1.653 & 0.147 & 0.034 & 2.893 & 0.168 & 1.832 & 0.000 & 0.251 & 0.004 & 15.304 \\
\hline 95.58 & $\begin{array}{l}7.184 \\
7776\end{array}$ & 0.816 & 0.371 & 0.030 & 0.001 & 1.708 & 0.141 & 0.032 & 2.717 & 0.129 & 1.871 & 0.000 & 0.238 & 0.004 & 15.288 \\
\hline $\begin{array}{l}97.22 \\
97.47\end{array}$ & $\begin{array}{l}7.767 \\
7.556\end{array}$ & 0.233 & 0.103 & 0.008 & 0.002 & 2.388 & 0.188 & 0.043 & $\begin{array}{r}2.268 \\
3.200\end{array}$ & 0.181 & 1.743 & 0.075 & 0.000 & 0.000 & 15.061 \\
\hline $\begin{array}{l}97.47 \\
96.54\end{array}$ & $\begin{array}{l}7.5522 \\
7.477\end{array}$ & $\begin{array}{l}0.448 \\
0.523\end{array}$ & $\begin{array}{l}0.132 \\
0.033\end{array}$ & $\begin{array}{l}0.058 \\
0.038\end{array}$ & $\begin{array}{l}0.009 \\
0.000\end{array}$ & $\begin{array}{l}1.245 \\
1.017\end{array}$ & $\begin{array}{l}0.329 \\
0.551\end{array}$ & $\begin{array}{l}0.022 \\
0.033\end{array}$ & $\begin{array}{l}3.205 \\
3.446\end{array}$ & $\begin{array}{l}0.233 \\
0.000\end{array}$ & $\begin{array}{l}1.628 \\
1.695\end{array}$ & $\begin{array}{l}0.139 \\
0.137\end{array}$ & 0.000 & $\begin{array}{l}0.000 \\
0.000\end{array}$ & $\begin{array}{l}15.108 \\
15000\end{array}$ \\
\hline 97.62 & 7.369 & 0.631 & $\begin{array}{l}0.033 \\
0.063\end{array}$ & 0.084 & $\begin{array}{l}0.000 \\
0.000\end{array}$ & $\begin{array}{l}1.017 \\
1.074\end{array}$ & $\begin{array}{l}0.551 \\
0.555\end{array}$ & $\begin{array}{l}0.043 \\
0.041\end{array}$ & $\begin{array}{l}3.496 \\
3.269\end{array}$ & $\begin{array}{l}0.000 \\
0.000\end{array}$ & $\begin{array}{l}1.695 \\
1.756\end{array}$ & $\begin{array}{l}0.137 \\
0.158\end{array}$ & $\begin{array}{l}0.000 \\
0.000\end{array}$ & $\begin{array}{l}0.000 \\
0.004\end{array}$ & $\begin{array}{l}15.000 \\
15.004\end{array}$ \\
\hline 96.86 & 7.067 & 0.933 & 0.170 & 0.042 & 0.000 & 0.829 & 0.994 & 0.028 & 2.853 & 0.000 & 1.764 & 0.320 & 0.000 & 0.004 & 15.004 \\
\hline 97.32 & 7.712 & 0.288 & 0.042 & 0.038 & 0.000 & 1.544 & 0.304 & 0.023 & 3.049 & 0.091 & 1.774 & 0.135 & 0.000 & 0.001 & 15.101 \\
\hline $\begin{array}{l}97.37 \\
0872\end{array}$ & 7.712 & 0.288 & 0.148 & 0.035 & 0.000 & 2.569 & 0.000 & 0.063 & 2.184 & 0.227 & 1.773 & 0.000 & 0.081 & 0.002 & $\begin{array}{r}15.079 \\
\end{array}$ \\
\hline $\begin{array}{l}98.20 \\
97.62\end{array}$ & $\begin{array}{r}7.525 \\
7.774\end{array}$ & $\begin{array}{l}0.475 \\
0.256\end{array}$ & 0.114 & 0.044 & 0.006 & 3.151 & 0.127 & 0.090 & 1.467 & 0.685 & 1.315 & 0.000 & 0.129 & 0.010 & 15.181 \\
\hline $\begin{array}{l}97.62 \\
97.62\end{array}$ & $\begin{array}{l}7.7444 \\
7.566\end{array}$ & $\begin{array}{l}0.256 \\
0.434\end{array}$ & $\begin{array}{l}0.099 \\
0.211\end{array}$ & $\begin{array}{l}0.035 \\
0.031\end{array}$ & 0.001 & $\begin{array}{l}1.987 \\
1995\end{array}$ & $\begin{array}{l}0.000 \\
\end{array}$ & 0.036 & 2.841 & 0.247 & 1.753 & 0.000 & $\begin{array}{l}0.091 \\
0.139\end{array}$ & 0.000 & $\begin{array}{l}15.089 \\
15.147\end{array}$ \\
\hline 98.88 & $\begin{array}{l}7.800 \\
7.837\end{array}$ & 0.163 & 0.066 & $\begin{array}{l}0.031 \\
0.014\end{array}$ & 0.007 & $\begin{array}{l}1.998 \\
3.406\end{array}$ & $\begin{array}{l}0.012 \\
0.000\end{array}$ & $\begin{array}{l}0.042 \\
0.078\end{array}$ & $\begin{array}{l}2.740 \\
\quad .434\end{array}$ & $\begin{array}{l}0.304 \\
0.353\end{array}$ & $\begin{array}{l}1.696 \\
1.647\end{array}$ & $\begin{array}{l}0.000 \\
0.000\end{array}$ & $\begin{array}{l}0.139 \\
0.062\end{array}$ & $\begin{array}{l}0.004 \\
0.006\end{array}$ & $\begin{array}{l}15.147 \\
15.068\end{array}$ \\
\hline 96.36 & 7.668 & 0.332 & 0.093 & 0.024 & 0.011 & 2.045 & 0.301 & 0.015 & 2.511 & $\begin{array}{l}0.3023 \\
0.022\end{array}$ & 1.833 & $\begin{array}{l}0.145 \\
0.145\end{array}$ & 0.000 & $\begin{array}{l}0.000 \\
0.025\end{array}$ & $\begin{array}{l}15.068 \\
15.124\end{array}$ \\
\hline 98.63 & 7.750 & 0.250 & 0.067 & 0.010 & 0.006 & 3.341 & 0.061 & 0.060 & 1.455 & 0.157 & 1.843 & 0.000 & 0.086 & 0.010 & 15.116 \\
\hline 96.08 & $\begin{array}{r}7.479 \\
77409\end{array}$ & 0.521 & 0.174 & 0.052 & 0.000 & 1.655 & 0.326 & 0.028 & 2.808 & 0.000 & 1.871 & 0.086 & 0.000 & 0.002 & 15.002 \\
\hline $\begin{array}{l}96.86 \\
97.81\end{array}$ & $\begin{array}{r}7.404 \\
7.444\end{array}$ & 0.596 & 0.102 & 0.045 & 0.017 & 2.770 & 0.584 & 0.045 & 1.436 & 0.372 & 1.423 & 0.205 & 0.000 & 0.008 & 15.201 \\
\hline $\begin{array}{l}97.01 \\
98.37\end{array}$ & $\begin{array}{l}7.448 \\
7.357\end{array}$ & $\begin{array}{l}0.552 \\
0.092\end{array}$ & $\begin{array}{l}0.172 \\
0.000\end{array}$ & 0.050 & 0.004 & $\begin{array}{r}3.350 \\
0.550\end{array}$ & $\begin{array}{r}0.000 \\
1.400\end{array}$ & 0.029 & $\begin{array}{l}1.395 \\
2.61\end{array}$ & 0.380 & 1.620 & 0.000 & 0.244 & $\begin{array}{l}0.032 \\
0.000\end{array}$ & $\begin{array}{l}15.276 \\
15000\end{array}$ \\
\hline 96.50 & $\begin{array}{l}7.3517 \\
7.627\end{array}$ & $\begin{array}{l}0.092 \\
0.373\end{array}$ & $\begin{array}{l}0.000 \\
0.081\end{array}$ & $\begin{array}{l}0.017 \\
0.009\end{array}$ & $\begin{array}{l}0.000 \\
0.000\end{array}$ & $\begin{array}{l}0.552 \\
2.015\end{array}$ & $\begin{array}{l}1.241 \\
0.343\end{array}$ & $\begin{array}{l}0.058 \\
0.039\end{array}$ & $\begin{array}{l}2.617 \\
2.550\end{array}$ & $\begin{array}{l}0.000 \\
0.000\end{array}$ & $\begin{array}{l}2.985 \\
1.891\end{array}$ & $\begin{array}{l}0.081 \\
0.072\end{array}$ & $\begin{array}{l}0.000 \\
0.000\end{array}$ & $\begin{array}{l}0.000 \\
0.004\end{array}$ & $\begin{array}{l}15.000 \\
15.004\end{array}$ \\
\hline 97.72 & 7.597 & 0.403 & 0.181 & 0.026 & 0.013 & 1.962 & 0.249 & 0.056 & 2.512 & 0.214 & $\begin{array}{l}1.891 \\
1.684\end{array}$ & 0.102 & $\begin{array}{l}0.000 \\
0.000\end{array}$ & $\begin{array}{l}0.004 \\
0.009\end{array}$ & $\begin{array}{l}15.004 \\
15.091\end{array}$ \\
\hline 98.40 & 7.481 & 0.519 & 0.252 & 0.022 & 0.017 & 2.076 & 0.032 & 0,041 & 2.560 & 0.325 & 1.675 & 0.000 & 0.161 & 0.013 & 15.185 \\
\hline $\begin{array}{l}96.07 \\
06.60\end{array}$ & $\begin{array}{l}7.462 \\
7.656\end{array}$ & $\begin{array}{l}0.538 \\
0325\end{array}$ & 0.223 & 0.038 & 0.000 & $\begin{array}{l}1.392 \\
1.002\end{array}$ & 0.435 & 0.011 & 2.901 & 0.000 & & 0.203 & 0.000 & 0.006 & \\
\hline 96.69 & $\begin{array}{l}7.665 \\
7.446\end{array}$ & $\begin{array}{l}0.335 \\
0.554\end{array}$ & 0.124 & 0.020 & 0.000 & 1.929 & 0.236 & 0.026 & 2.785 & 0.000 & 1,810 & 0.069 & 0.000 & 0.004 & 15.004 \\
\hline $\begin{array}{l}99.14 \\
97.40\end{array}$ & $\begin{array}{l}7.446 \\
7.582\end{array}$ & $\begin{array}{l}0.554 \\
0.418\end{array}$ & 0.055 & 0.004 & 0.009 & 1.661 & $\begin{array}{r}0.255 \\
0.42\end{array}$ & 0.033 & $\begin{array}{r}2.982 \\
2.355\end{array}$ & 0.160 & 1.840 & 0.000 & $\begin{array}{l}0.209 \\
0.109\end{array}$ & 0.017 & 15.311 \\
\hline 97.75 & $\begin{array}{l}7,582 \\
7.319\end{array}$ & $\begin{array}{l}0.468 \\
0.681\end{array}$ & $\begin{array}{l}0.126 \\
0.239\end{array}$ & $\begin{array}{l}0.025 \\
0.010\end{array}$ & $\begin{array}{l}0.001 \\
0.000\end{array}$ & $\begin{array}{l}2.381 \\
1.949\end{array}$ & $\begin{array}{l}0.043 \\
0.139\end{array}$ & $\begin{array}{l}0.069 \\
0.058\end{array}$ & $\begin{array}{l}2.355 \\
2.606\end{array}$ & $\begin{array}{l}0.569 \\
0.322\end{array}$ & $\begin{array}{l}1.431 \\
1.678\end{array}$ & $\begin{array}{l}0.000 \\
0.000\end{array}$ & $\begin{array}{l}0.199 \\
0.282\end{array}$ & $\begin{array}{l}0.000 \\
0.002\end{array}$ & $\begin{array}{l}15.213 \\
15.330\end{array}$ \\
\hline 97.19 & 7.740 & 0.260 & 0.077 & 0.022 & 0.016 & 2.062 & $\begin{array}{l}0.139 \\
0.248\end{array}$ & $\begin{array}{l}0.0058 \\
0.092\end{array}$ & $\begin{array}{l}2.2006 \\
2.482\end{array}$ & $\begin{array}{l}0.322 \\
1.223\end{array}$ & $\begin{array}{l}1.678 \\
0.647\end{array}$ & $\begin{array}{l}0.000 \\
0.130\end{array}$ & $\begin{array}{l}0.282 \\
0.000\end{array}$ & 0.0042 & $\begin{array}{l}15.08305 \\
15.085\end{array}$ \\
\hline 96.47 & 7.616 & 0.384 & 0.174 & 0.011 & 0.001 & 1.686 & 0.377 & 0.065 & 2.685 & 0.697 & 1.111 & 0.192 & 0.000 & 0.002 & \\
\hline $\begin{array}{l}97.61 \\
02501\end{array}$ & $\begin{array}{l}7.570 \\
7.778\end{array}$ & 0.430 & 0.133 & 0.023 & 0.003 & 1.605 & 0.404 & 0.063 & 2.770 & 0.370 & 1.470 & 0.160 & 0.000 & 0.004 & 15.137 \\
\hline $\begin{array}{l}93.50 \\
98.42\end{array}$ & $\begin{array}{r}7.782 \\
7.249\end{array}$ & 0.218 & $\begin{array}{l}0.029 \\
0.245\end{array}$ & 0.023 & 0.001 & $\begin{array}{l}1.644 \\
1.504\end{array}$ & 0.231 & 0.088 & 2.785 & 0.000 & 1,916 & 0.084 & 0.009 & 0.003 & 15.012 \\
\hline 98.42 & $\begin{array}{l}7.248 \\
7.473\end{array}$ & $\begin{array}{l}0.752 \\
0.527\end{array}$ & $\begin{array}{l}0.245 \\
0.254\end{array}$ & $\begin{array}{l}0.041 \\
0.047\end{array}$ & 0.093 & $\begin{array}{l}1.501 \\
1.698\end{array}$ & 0.098 & 0.031 & $\begin{array}{r}2.991 \\
-2907\end{array}$ & 0.152 & $\begin{array}{l}1.848 \\
1.978\end{array}$ & 0.000 & 0.232 & 0.002 & $\begin{array}{l}15.266 \\
15.110\end{array}$ \\
\hline $\begin{array}{l}99.13 \\
95.50\end{array}$ & $\begin{array}{l}7.473 \\
7.364\end{array}$ & $\begin{array}{l}0.327 \\
0.636\end{array}$ & $\begin{array}{l}0.254 \\
0.233\end{array}$ & $\begin{array}{l}0.047 \\
0.065\end{array}$ & $\begin{array}{l}0.000 \\
0.000\end{array}$ & $\begin{array}{l}1.693 \\
1.183\end{array}$ & $\begin{array}{l}0.011 \\
0.525\end{array}$ & $\begin{array}{l}0.023 \\
0.022\end{array}$ & $\begin{array}{l}2.972 \\
2.971\end{array}$ & $\begin{array}{l}0.203 \\
0.120\end{array}$ & $\begin{array}{l}1.797 \\
1.616\end{array}$ & $\begin{array}{l}0.000 \\
0.264\end{array}$ & $\begin{array}{l}0.164 \\
0.000\end{array}$ & $\begin{array}{l}0.004 \\
0.04\end{array}$ & $\begin{array}{l}15.177 \\
15.85\end{array}$ \\
\hline 98.50 & 7.661 & 0.339 & 0.111 & 0.034 & 0.000 & $\begin{array}{l}1.706 \\
1.703\end{array}$ & 0.266 & 0.040 & 2.842 & $\begin{array}{l}0.120 \\
0.299\end{array}$ & $\begin{array}{l}1.616 \\
1.593\end{array}$ & $\begin{array}{l}0.264 \\
0.109\end{array}$ & 0.0000 & $\begin{array}{l}0.004 \\
0.004\end{array}$ & $\begin{array}{l}15.185 \\
15.09\end{array}$ \\
\hline 94.27 & 7.385 & 0.615 & 0.207 & 0.031 & 0.005 & 1.467 & 0.592 & 0.042 & 2.656 & 0.198 & 1.548 & 0.254 & 0.000 & $\begin{array}{l}0.004 \\
0.004\end{array}$ & $\begin{array}{l}15.200 \\
\end{array}$ \\
\hline 97.96 & 7.602 & 0.398 & 0.131 & 0.029 & 0.001 & 1.550 & 0.327 & 0.02 & 2.933 & 0.148 & 1.7 & 0.120 & 0.000 & 0,000 & 15.108 \\
\hline 97.5 & 7.497 & 0.503 & 0.016 & 0.031 & 0.009 & 193 & 0.23 & & 277 & 0462 & 15 & & 0 & 0.000 & 15.259 \\
\hline 97.69 & 7.714 & 0.286 & 0.001 & 0.017 & 0.000 & 1.686 & 0.173 & 0.044 & 3080 & 0.368 & 16 & 0.000 & & 0.004 & 15.136 \\
\hline 96.18 & 7,470 & 0.530 & 0.054 & 0.058 & 0.025 & 1.580 & 0.221 & 0.028 & 3.035 & 0.063 & 1.937 & 0.000 & 0.115 & 0.000 & 15.188 \\
\hline 98.67 & 6.715 & 1.249 & 0.000 & 0.068 & 0.000 & 0.177 & 1.585 & 0.026 & 3.093 & 0.000 & 1.668 & 0.420 & 0.000 & 0.022 & 15.022 \\
\hline 96.50 & 7.374 & 0.626 & 0.199 & 0.055 & 0.000 & 1.194 & 0.492 & 0.024 & 3.036 & 0.000 & 1.806 & 0.180 & 0.000 & 0.006 & 15.006 \\
\hline 95. & 7.195 & 0.786 & 0.000 & 0.021 & 0.000 & 1.516 & 0.927 & 0.046 & 2.682 & 0.000 & 1.675 & 0.151 & 0.000 & 0.004 & \\
\hline 96.50 & 7.374 & 0.626 & 0.199 & 0.055 & 0.000 & 1.194 & 0.492 & 0.0 & 3.036 & 0.000 & 1.806 & 0.180 & 0.000 & 0.006 & \\
\hline 96.50 & 7.128 & 0.872 & 1.230 & 0.000 & 0.000 & 0.998 & 0.003 & & 1.927 & 0.000 & & & & & \\
\hline $\begin{array}{l}96.25 \\
06.37\end{array}$ & $\begin{array}{l}7.476 \\
-7502\end{array}$ & 0.524 & $\begin{array}{l}0.179 \\
0274\end{array}$ & 0.026 & 0.013 & $\begin{array}{l}1.529 \\
1.65\end{array}$ & 0.416 & 0.033 & 2.900 & 0.000 & 1.775 & 0.125 & 0.018 & 0.006 & 15.024 \\
\hline $\begin{array}{l}96.37 \\
98.40\end{array}$ & $\begin{array}{l}7.521 \\
7.757\end{array}$ & $\begin{array}{l}0.479 \\
0.243\end{array}$ & $\begin{array}{l}0.224 \\
0.170\end{array}$ & $\begin{array}{l}0.028 \\
0.018\end{array}$ & $\begin{array}{l}0.004 \\
0.005\end{array}$ & $\begin{array}{l}1.652 \\
2.052\end{array}$ & $\begin{array}{l}0.346 \\
0.11\end{array}$ & $\begin{array}{l}0.033 \\
0.014\end{array}$ & $\begin{array}{r}2.883 \\
2621\end{array}$ & $\begin{array}{l}0.000 \\
0.000\end{array}$ & $\begin{array}{l}1.693 \\
1914\end{array}$ & $\begin{array}{l}0.138 \\
0.888\end{array}$ & 0.0155 & 0.004 & $\begin{array}{l}15.019 \\
15.044\end{array}$ \\
\hline $\begin{array}{r}98.40 \\
100.09\end{array}$ & 7.071 & $\begin{array}{l}0.243 \\
0.929\end{array}$ & $\begin{array}{l}0.170 \\
0.067\end{array}$ & $\begin{array}{l}0.018 \\
0.210\end{array}$ & 0.000 & $\begin{array}{l}2.0022 \\
1.746\end{array}$ & $\begin{array}{l}0.119 \\
0.055\end{array}$ & $\begin{array}{l}0.014 \\
0.022\end{array}$ & $\begin{array}{l}2.621 \\
2.900\end{array}$ & $\begin{array}{l}0.000 \\
0.117\end{array}$ & $\begin{array}{l}1.914 \\
1.883\end{array}$ & $\begin{array}{l}0.088 \\
0.000\end{array}$ & $\begin{array}{l}0.000 \\
0.366\end{array}$ & $\begin{array}{l}0.002 \\
0.020\end{array}$ & $\begin{array}{l}15.041 \\
15.405\end{array}$ \\
\hline & 7.793 & 0.207 & 0.147 & 0.012 & 0.007 & 2.144 & 0.101 & 0.0 & 2.535 & 0.121 & 1.808 & 0.071 & & & \\
\hline 98.21 & 7.490 & 0.5 & 0.1 & 0.023 & 0.0 & 1.6 & & 0.0 & 2.696 & 0.1 & 1.7 & 0.160 & & 0.0 & \\
\hline 94.40 & $\begin{array}{r}7.443 \\
7.20\end{array}$ & 0.557 & 0.270 & 0.018 & 0.012 & 1.401 & 0.0 & 0.016 & 3.2 & 0.165 & 1.835 & 0.000 & & & \\
\hline $\begin{array}{l}99.28 \\
95.67\end{array}$ & $\begin{array}{l}7.319 \\
6.976\end{array}$ & $\begin{array}{l}0.681 \\
1024\end{array}$ & $\begin{array}{l}0.328 \\
0.093\end{array}$ & $\begin{array}{l}0.043 \\
0.145\end{array}$ & $\begin{array}{l}0.000 \\
0.000\end{array}$ & $\begin{array}{l}1.875 \\
1.45\end{array}$ & 0.014 & 0.032 & $\begin{array}{r}2.708 \\
3\end{array}$ & $\begin{array}{l}0.161 \\
0.358\end{array}$ & 1.839 & 0.000 & $\begin{array}{l}0.249 \\
0 \leq 501\end{array}$ & 0.004 & $\begin{array}{l}15.257 \\
15.557\end{array}$ \\
\hline $\begin{array}{l}95,67 \\
99.06\end{array}$ & $\begin{array}{l}6.976 \\
7.208\end{array}$ & $\begin{array}{l}1.024 \\
0.792\end{array}$ & $\begin{array}{l}0.093 \\
0.048\end{array}$ & $\begin{array}{l}0.145 \\
0.072\end{array}$ & 0.000 & $\begin{array}{l}1.454 \\
0720\end{array}$ & $\begin{array}{l}0.127 \\
0.882\end{array}$ & 0.009 & $\begin{array}{l}3.172 \\
33242\end{array}$ & 0.358 & $\begin{array}{l}1.642 \\
1.675\end{array}$ & $\begin{array}{r}0.000 \\
0.325\end{array}$ & $\begin{array}{l}0.501 \\
0.5000\end{array}$ & $\begin{array}{l}0.013 \\
0.029\end{array}$ & $\begin{array}{l}15.557 \\
15.320\end{array}$ \\
\hline 99.05 & 7.332 & 0.668 & $\begin{array}{l}0.276 \\
0.276\end{array}$ & 0.056 & 0.022 & $\begin{array}{l}0.729 \\
1.770\end{array}$ & $\begin{array}{l}0.8822 \\
0.025\end{array}$ & $\begin{array}{l}0.014 \\
0.032\end{array}$ & $\begin{array}{l}3.241 \\
2820\end{array}$ & $\begin{array}{l}0.000 \\
0.217\end{array}$ & $\begin{array}{l}1.675 \\
1.783\end{array}$ & $\begin{array}{l}0.325 \\
0.000\end{array}$ & $\begin{array}{l}0.000 \\
0.226\end{array}$ & $\begin{array}{l}0.029 \\
0.007\end{array}$ & $\begin{array}{l}15.322 \\
15.242\end{array}$ \\
\hline 97. & 7.027 & 0.9 & 0.2 & 0.112 & 0.0 & 1.5 & 0.170 & 0.0 & 2.9 & 0.268 & 1.732 & 0.000 & & 0.0 & \\
\hline $\begin{array}{l}98.88 \\
05.30\end{array}$ & 7.071 & & 0.2 & & 0.0 & 1.5 & 0.1 & 0.0 & 3.0 & 0.3 & 1.6 & 0.0 & & & \\
\hline $\begin{array}{l}95.39 \\
07.19\end{array}$ & 7.450 & 0.5 & 0.0 & 0.0 & 0.007 & 1.4 & & & 3.2 & 0.332 & 1.6 & & & & \\
\hline $\begin{array}{l}97.14 \\
97.37\end{array}$ & $\begin{array}{r}6.913 \\
77696\end{array}$ & $\begin{array}{l}1.087 \\
0.337\end{array}$ & 0.276 & 0.044 & 0.000 & 1.489 & 0.119 & 0.019 & 3.053 & 0.349 & 1.651 & 0.000 & 0.594 & 0.009 & 15 \\
\hline $\begin{array}{l}97.37 \\
99.21\end{array}$ & $\begin{array}{l}7.6699 \\
7.796\end{array}$ & $\begin{array}{l}0.331 \\
0.204\end{array}$ & $\begin{array}{l}0.016 \\
0.050\end{array}$ & $\begin{array}{l}0.040 \\
0.027\end{array}$ & 0.005 & $\begin{array}{r}2.109 \\
2.244\end{array}$ & 0.066 & 0.049 & $\begin{array}{l}2.716 \\
26604\end{array}$ & 0.504 & $\begin{array}{l}1.496 \\
1775\end{array}$ & 0.000 & 0.154 & 0.011 & $\begin{array}{l}15.187 \\
15.1\end{array}$ \\
\hline 98.05 & $\begin{array}{l}7.796 \\
7.735\end{array}$ & $\begin{array}{l}0.2 \\
0.2\end{array}$ & $\begin{array}{l}0.1 \\
0.1\end{array}$ & $\begin{array}{l}0.027 \\
0.035\end{array}$ & $\begin{array}{l}0.002 \\
0.000\end{array}$ & $\begin{array}{l}2.243 \\
1.697\end{array}$ & $\begin{array}{l}0.0 \\
0.2\end{array}$ & $\begin{array}{l}0.037 \\
0.020\end{array}$ & $\begin{array}{l}2.2640 \\
2.914\end{array}$ & $\begin{array}{l}0.225 \\
0.190\end{array}$ & $\begin{array}{l}1.775 \\
1.670\end{array}$ & $\begin{array}{l}0.000 \\
0.140\end{array}$ & $\begin{array}{l}0.102 \\
0.000\end{array}$ & 0.0 & $\begin{array}{l}15.102 \\
15.066\end{array}$ \\
\hline 95 . & 7.133 & 0.8 & & 0.0 & 0.0 & 2.2 & & 0.0 & 2.2 & & 1.6 & & & 0.0 & \\
\hline & 7.543 & 0.4 & 0.2 & & 0.0 & 1.8 & & 0.0 & 2.8 & & 1.8 & 0. & & & \\
\hline 97.10 & $\begin{array}{r}7.403 \\
7.156\end{array}$ & 0.5 & 0.195 & 0.0 & 0.012 & & & & & & 1.8 & & & & \\
\hline $\begin{array}{l}97.68 \\
06>0\end{array}$ & $\begin{array}{l}7.156 \\
7440\end{array}$ & 0.8 & $\begin{array}{l}0.346 \\
0219\end{array}$ & 0.053 & 0.000 & 1.767 & $\begin{array}{l}0.178 \\
0537\end{array}$ & 0.049 & 2.6 & 0.407 & $\begin{array}{l}1.593 \\
1540\end{array}$ & 0. & 0.2 & 0.6 & \\
\hline $\begin{array}{l}96.2 \\
95 .\end{array}$ & $\begin{array}{l}7.402 \\
7.197\end{array}$ & 0.5 & $\begin{array}{l}0.219 \\
0.185\end{array}$ & $\begin{array}{l}0.027 \\
0.052\end{array}$ & 0.000 & $\begin{array}{l}1.163 \\
0.9953\end{array}$ & $\begin{array}{l}0.537 \\
0.774\end{array}$ & 0.047 & $\begin{array}{l}3.241 \\
2.995\end{array}$ & 0.000 & $\begin{array}{l}1.549 \\
1800\end{array}$ & $\begin{array}{l}0.216 \\
0.272\end{array}$ & $\begin{array}{l}0.000 \\
0.000\end{array}$ & 0.004 & \\
\hline & 7.38 & 0.5 & & & & & & & $\begin{array}{l}2.9 \\
3.2\end{array}$ & & $\begin{array}{l}1.8 \\
1.8\end{array}$ & & & & \\
\hline & 6.663 & 1.2 & & & & & & & & & 1.8 & & & & \\
\hline 95. & 7.099 & 0.6 & 0.0 & & & & & 0.0 & 1.8 & & & 0.2 & & & \\
\hline & 7335 & 0.6 & 0.1 & & 0.0 & & & & & & & & & & \\
\hline & 558 & 0.4 & 0.1 & 01 & 0. & 1.9 & 0. & 0.0 & 2.8 & 03 & & & 0.1 & 0 . & \\
\hline & 7.6 & & & & & 2. & & & 2.3 & & 1.4 & 5 & & & \\
\hline & 7.350 & & & & & 1.7 & & & 2.8 & 3 & 1.7 & & & & \\
\hline & 7.159 & & & & & & & & 2.5 & & $i .5$ & 0 . & & & \\
\hline & 7.652 & 0.3 & 0.1 & & 0.0 & 1.65 & & & 2.8 & & 1.6 & & & & \\
\hline 98.47 & 7.196 & 0.804 & 0.0 & 0.1 & 0.024 & 1.1 & 0.734 & 0.0 & 2.876 & & 1.6 & 0.259 & & & \\
\hline 97.72 & $\begin{array}{l}7.343 \\
-7.163\end{array}$ & 0.657 & 0.091 & 0.083 & 0.009 & 1.274 & 0.622 & 0.034 & 2.888 & 0.155 & 1.599 & 0.246 & 0.000 & 0.017 & 15.222 \\
\hline $\begin{array}{l}97.9 \\
072 .\end{array}$ & $\begin{array}{l}7.163 \\
7466\end{array}$ & 0.837 & 0.411 & 0.025 & 0.000 & 2.013 & 0.039 & 0.050 & $\begin{array}{r}2.462 \\
2.055\end{array}$ & 0.284 & 1.716 & 0.000 & 0.325 & 0.0011 & $\begin{array}{l}15.349 \\
15000\end{array}$ \\
\hline $\begin{array}{l}97.31 \\
962\end{array}$ & 7.521 & 0.4 & $\begin{array}{l}0.187 \\
0.139\end{array}$ & $\begin{array}{l}0.040 \\
0.032\end{array}$ & 0.000 & $\begin{array}{l}1.422 \\
1.551\end{array}$ & $\begin{array}{l}0.418 \\
0.406\end{array}$ & $\begin{array}{l}0.033 \\
0.031\end{array}$ & $\begin{array}{l}2.955 \\
2.806\end{array}$ & $\begin{array}{l}0.000 \\
0.000\end{array}$ & $\begin{array}{l}1.788 \\
1.905\end{array}$ & $\begin{array}{l}0.156 \\
0.130\end{array}$ & $\begin{array}{l}0.000 \\
0.000\end{array}$ & $\begin{array}{l}0.003 \\
0.001\end{array}$ & $\begin{array}{r}15.003 \\
15.001\end{array}$ \\
\hline 96.46 & 7.218 & 0.782 & 0.230 & 0.050 & 0.000 & 1.215 & 0.668 & 0.032 & 2.783 & 0.000 & 1.798 & 0.223 & 0.000 & 0.007 & 15.007 \\
\hline
\end{tabular}

other secondary minerals. Whether prehnite is in equilibrium with epidote and/or actinolite is not clear. Prehnite also occurs in later veins associated with epidote. It partly replaces plagioclase, particularly when in alteration patches.

Some representative analyses of prehnite from Legs 137/140 are presented in Table 8. Compositions are similar to those of Leg 111 prehnites coexisting with epidote and laumontite reported by Ishizuka (1989), although their $\mathrm{XFe}^{3+}$ ratio can still be lower. Leg 83 prehnites also generally have lower $\mathrm{XFe}^{3+}$ than Leg 111 ones. This difference may be because the prehnite we analyzed in Legs $137 / 140$ rocks is not associated with either epidote, laumontite, or actinolite, as it is in Leg 111 rocks. This relatively low $\mathrm{XFe}^{3+}$ suggests that prehnite from Legs 
Table 2 (continued).

\begin{tabular}{|c|c|c|c|c|c|c|c|c|c|c|c|c|c|c|c|c|}
\hline $\begin{array}{l}\text { Core, section, } \\
\text { interval }(\mathrm{cm})\end{array}$ & $\begin{array}{c}\text { Expandable } \\
\text { depth }\end{array}$ & Analysis & Occurrence & Mineral & $\mathrm{SiO}_{2}$ & $\mathrm{Al}_{2} \mathrm{O}_{3}$ & $\mathrm{FeO}_{\mathrm{t}}$ & $\mathrm{MnO}$ & $\mathrm{MgO}$ & $\mathrm{CaO}$ & $\mathrm{Na}_{2} \mathrm{O}$ & $\mathrm{K}_{2} \mathrm{O}$ & $\mathrm{TiO}_{2}$ & $\mathrm{Cr}_{2} \mathrm{O}_{3}$ & $\mathrm{ZnO}$ & $\mathrm{Cl}$ \\
\hline \multirow{6}{*}{$236 \mathrm{R}-1,5-9$} & 1977.4 & A16 & ground. & Act & 52.23 & 3.09 & 18.15 & 0.38 & 12.78 & 11.10 & 0.39 & 0.02 & 0.22 & 0.00 & 0.08 & 0.05 \\
\hline & 1981.0 & A27 & vein wall & $\mathrm{Fe}-\mathrm{Act}$ & 51.85 & 0.85 & 24.59 & 0.48 & 8.14 & 9.50 & 0.62 & 0.00 & 0.26 & 0.02 & 0.00 & 0.05 \\
\hline & 1981.0 & A28 & vein wall & Act & 51.69 & 3.49 & 16.37 & 0.27 & 12.03 & 12.26 & 0.40 & 0.00 & 0.27 & 0.03 & 0.00 & 0.05 \\
\hline & 1981.0 & $\mathrm{~A} 30$ & ground. & Act-Hbl & 51.71 & 4.86 & 13.17 & 0.22 & 14.74 & 10.62 & 0.68 & 0.02 & 0.32 & 0.01 & 0.06 & 0.06 \\
\hline & 1981.0 & A38 & vein & $\mathrm{Act}-\mathrm{Hbl}$ & 50.35 & 3.90 & 17.19 & 0.28 & 12.51 & 12.15 & 0.47 & 0.03 & 0.29 & 0.02 & 0.07 & 0.03 \\
\hline & 1981.0 & A46 & vein & Act & 51.56 & 2.86 & 15.82 & 0.25 & 13.37 & 11.54 & 0.48 & 0.00 & 0.14 & 0.05 & 0.00 & 0.15 \\
\hline $236 \mathrm{R}-1,30-32$ & 1981.8 & B3135 & vein & Act & 52.94 & 2.80 & 16.44 & 0.19 & 13.78 & 11.62 & 0.34 & 0.02 & 0.27 & 0.11 & 0.15 & 0.00 \\
\hline \multirow[t]{2}{*}{$236 \mathrm{R}-1,57-60$} & 1982.5 & B3084 & vein & Act-Hbl & 50.84 & 5.51 & 14.61 & 0.10 & 14.40 & 11.22 & 0.80 & 0.06 & 0.39 & 0.06 & 0.28 & 0.05 \\
\hline & 1982.5 & B3085 & ground. & $\mathrm{Act}-\mathrm{Hbl}$ & 48.78 & 3.69 & 14.13 & 0.29 & 14.47 & 8.69 & 0.63 & 0.05 & 0.18 & 0.39 & 0.09 & 0.25 \\
\hline \multirow{5}{*}{$236 R-1,67-68$} & 1982.8 & V1005 & vein & Edenite & 46.07 & 8.15 & 15.40 & 0.13 & 13.65 & 11.08 & 2.35 & 0.07 & 0.28 & 0.00 & 0.05 & 0.13 \\
\hline & 1982.8 & V1008 & vein & $\mathrm{Fe}-\mathrm{Act}$ & 52.61 & 0.82 & 27.63 & 0.26 & 7.43 & 11.00 & 0.31 & 0.00 & 0.06 & 0.00 & 0.00 & 0.05 \\
\hline & 1982.8 & V1014 & vein & Act & 54.60 & 2.06 & 14.55 & 0.27 & 15.99 & 9.81 & 1.12 & 0.03 & 0.23 & 0.00 & 0.14 & 0.03 \\
\hline & 1982.8 & V1016 & vein wall & $\mathrm{Act}-\mathrm{Hbl}$ & 51.64 & 4.23 & 15.80 & 0.28 & 13.55 & 11.41 & 0.67 & 0.03 & 0.28 & 0.01 & 0.02 & 0.05 \\
\hline & 1982.8 & V1017 & vein wall & Act & 52.87 & 3.79 & 15.91 & 0.36 & 14.33 & 10.98 & 0.66 & 0.02 & 0.19 & 0.06 & 0.00 & 0.11 \\
\hline \multirow[t]{3}{*}{$237 \mathrm{R}-1,24-26$} & 1991.1 & B3003 & vein & Act & 54.19 & 1.73 & 16.58 & 0.31 & 13.80 & 11.68 & 0.24 & 0.00 & 0.16 & 0.09 & 0.00 & 0.06 \\
\hline & 1991.1 & A 102 & halo & Act & 53.17 & 3.17 & 12.53 & 0.11 & 16.17 & 11.86 & 0.48 & 0.03 & 0.09 & 0.00 & 0.00 & 0.00 \\
\hline & 1991.1 & A 103 & halo & Act-Hbl & 51.07 & 5.56 & 12.92 & 0.23 & 14.71 & 11.45 & 0.77 & 0.00 & 0.32 & 0.00 & 0.00 & 0.01 \\
\hline
\end{tabular}

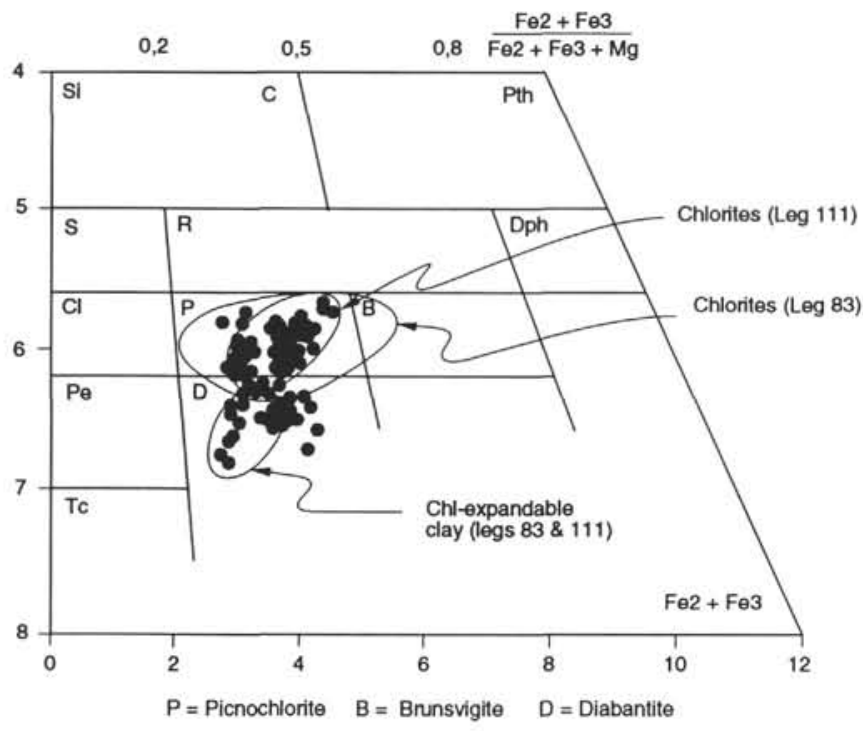

Figure 7. Molar compositions of Legs $137 / 140$ chlorites and mixed-layer chlorite-smectites. Fields of chlorites and mixed-layer clays from Leg 83 and Leg 111 rocks are shown for comparison (Alt et al., 1985, 1989b; Laverne, 1987b).

$137 / 140$ formed at higher $\mathrm{T}$ or lower oxygen fugacity than that of Leg 111.

\section{Zeolites}

Zeolites are minor phases in Leg 137/140 rocks. Zeolites occur in the central part of some actinolitic veins, frequently with epidote and/or very thin amphibole needles and/or prehnite, inside tension gashes opened within actinolite crystals filling the veins in Core 186 (Tartarotti et al., this volume), as a late phase in amygdules, and as partial replacement of either fresh or previously altered plagioclase. Different zeolites could not be distinguished optically, but different compositions (laumontite and heulandite [or stilbite?]) were identified by microprobe analyses (Table 9). Both of these zeolites occur in all of the above sites.

\section{Titanite}

Titanite is common in alteration halos around veins and in alteration patches. It occurs as anhedral to euhedral, sometimes granular,

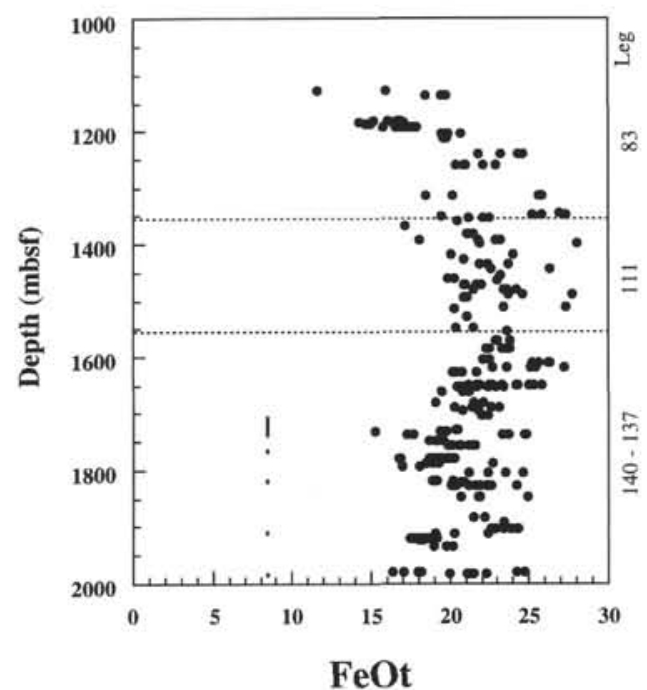

Figure 8. Plot of total FeO vs, depth for chlorite and mixed-layer chlorite-smectite for the complete sheeted dikes section of Hole 504B. Note that low Fe contents correspond to depth zones where fresh olivine relicts (bold line) occur.

crystals partly or completely replacing titanomagnetite. Its composition is fairly constant (Table 10), and is in the range of composition of Leg 83 and Leg 111 titanite (Fig. 9). Most of Hole 504B titanite analyses plot in the composition field of greenschist to amphibolite facies rocks from East Taiwan ophiolites (Liou and Ernst, 1979).

\section{Anhydrite}

Anhydrite occurs as a partial replacement of plagioclase in some highly altered patches, and in veins where quartz, epidote, prehnite, and laumontite also occur. Anhydrite apparently formed later than the other minerals but, as for the other minerals, the presence of anhydrite reflects the high permeability of these rocks, even after the crystallization of the first stage alteration minerals.

\section{Apatite}

Secondary apatite is a common accessory mineral that forms fine acicular crystals within actinolite veins, and within the interstitial actinolite occurring in vein halos. Secondary apatite has also been identified in amphibole replacing rims of clinopyroxene in Leg 111 dike samples (J. Alt, unpubl. data). 
Table 2 (continued).

\begin{tabular}{|c|c|c|c|c|c|c|c|c|c|c|c|c|c|c|c|c|c|}
\hline Total & $\mathrm{Si}$ & $\mathrm{Al}^{\mathrm{IV}}$ & $\mathrm{Al}^{\mathrm{VI}}$ & $\mathrm{T}$ & $\mathrm{Cr}$ & $\mathrm{Fe}^{2+}$ & $\mathrm{Fe}^{3+}$ & $\mathrm{Mn}$ & $\mathrm{Mg}$ & $\mathrm{MgM} 4$ & $\mathrm{Ca}$ & $\mathrm{NaM}_{4}$ & $\mathrm{NaA}$ & $\mathrm{K}$ & Total & $\mathrm{xMg}$ & $(\mathrm{Na}+\mathrm{K}) \mathrm{A}$ \\
\hline 98.48 & 7.589 & 0.411 & 0.118 & 0.023 & 0.000 & 2207 & 0.015 & 0.000 & 2788 & 0.000 & 1741 & 0.000 & 0.11 & 0.004 & 15.115 & 0.60 & 0.115 \\
\hline 96.37 & 7.972 & 0.028 & 0.126 & 0.030 & 0.002 & 3.138 & 0.025 & 0.063 & $\begin{array}{l}2.180 \\
1866\end{array}$ & 0.000 & 1566 & 0.185 & 0000 & 0.000 & 15,000 & 0.37 & 0.000 \\
\hline 96.87 & 7.615 & 0.385 & 0.222 & 0.030 & 0.004 & 1.805 & 0.212 & 0.034 & 2.642 & 0.000 & $\begin{array}{l}1.9360 \\
\end{array}$ & 0.114 & 0.000 & 0.000 & 15.000 & 0.59 & 0.000 \\
\hline 96.47 & 7.490 & 0.510 & 0.320 & 0.035 & 0.001 & 1.289 & 0.307 & 0.027 & 3.182 & 0.000 & 1.648 & 0.191 & 0.000 & 0.004 & 15.004 & 0.71 & 0.004 \\
\hline 97.29 & 7.377 & 0.623 & 0.052 & 0.032 & 0.002 & 1.473 & 0.634 & 0.035 & 2.732 & 0.000 & 1.907 & 0.134 & 0.000 & 0.006 & 15.006 & 0.65 & 0.006 \\
\hline 96.21 & 7.601 & 0.399 & 0.099 & 0.016 & 0.006 & 1.550 & $0.40 \mathrm{t}$ & 0.031 & 2.937 & 0.000 & 1.823 & 0.138 & 0.000 & 0.000 & & 0.65 & 0.000 \\
\hline 98.66 & 7.678 & 0.322 & $\begin{array}{l}0.156 \\
0\end{array}$ & 0.029 & 0.013 & 1.994 & 0.000 & 0.023 & 2.784 & 0.194 & 1.806 & 0.000 & 0.096 & 0.004 & 15.098 & 0.60 & 0.100 \\
\hline $\begin{array}{r}98.32 \\
0164\end{array}$ & $\begin{array}{l}7.371 \\
7487\end{array}$ & 0.629 & $\begin{array}{l}0.312 \\
0.154\end{array}$ & 0.043 & 0.007 & 1.771 & 0.000 & 0.012 & 2.855 & 0.257 & 1.743 & 0.000 & 0.225 & 0.011 & $\begin{array}{l}15.232 \\
\end{array}$ & 0.64 & 0.236 \\
\hline $\begin{array}{l}91.64 \\
97.36\end{array}$ & $\begin{array}{l}7.487 \\
6836\end{array}$ & $\begin{array}{l}0.513 \\
1164\end{array}$ & $\begin{array}{l}0.154 \\
0.261\end{array}$ & $\begin{array}{l}0.021 \\
0.023\end{array}$ & 0.047 & $\begin{array}{l}1.365 \\
1.760\end{array}$ & 0.448 & 0.038 & 2.927 & 0.384 & 1.429 & 0.187 & $\begin{array}{l}0.000 \\
0.676\end{array}$ & $\begin{array}{r}0.010 \\
\end{array}$ & 15.157 & 0.71 & $\begin{array}{l}0.010 \\
0.690\end{array}$ \\
\hline $\begin{array}{r}97.36 \\
100.17\end{array}$ & $\begin{array}{l}6.836 \\
7.859\end{array}$ & $\begin{array}{l}1.164 \\
0.141\end{array}$ & $\begin{array}{l}0.261 \\
0.04\end{array}$ & $\begin{array}{l}0.031 \\
0.007\end{array}$ & 0.000 & $\begin{array}{l}1.760 \\
3.329\end{array}$ & 0.151 & $\begin{array}{l}0.016 \\
0.033\end{array}$ & $\begin{array}{l}2.780 \\
1505\end{array}$ & $\begin{array}{l}0.239 \\
0.150\end{array}$ & $\begin{array}{l}1.766 \\
1776\end{array}$ & $\begin{array}{l}0.000 \\
0.090\end{array}$ & $\begin{array}{l}0.676 \\
0.000\end{array}$ & $\begin{array}{l}0.013 \\
0.000\end{array}$ & $\begin{array}{l}15.741 \\
5.070\end{array}$ & $\begin{array}{l}0.63 \\
0.34\end{array}$ & $\begin{array}{l}0.689 \\
0.000\end{array}$ \\
\hline 98.83 & $\begin{array}{l}7.859 \\
7.718\end{array}$ & $\begin{array}{l}0.141 \\
0.282\end{array}$ & $\begin{array}{l}0.064 \\
0.061\end{array}$ & $\begin{array}{l}0.0027 \\
0.024\end{array}$ & $\begin{array}{l}0.000 \\
0.000\end{array}$ & $\begin{array}{l}3.239 \\
1.247\end{array}$ & $\begin{array}{l}0.2 \\
0.4\end{array}$ & $\begin{array}{l}0.033 \\
0.032\end{array}$ & $\begin{array}{l}1.505 \\
3.162\end{array}$ & $\begin{array}{l}0.150 \\
0.207\end{array}$ & $\begin{array}{l}1.761 \\
1.486\end{array}$ & $\begin{array}{l}0.090 \\
0.307\end{array}$ & $\begin{array}{l}0.000 \\
0.000\end{array}$ & 0.005 & $\begin{array}{l}15.07001 \\
15.161\end{array}$ & $\begin{array}{l}0.34 \\
0.73\end{array}$ & 0.005 \\
\hline 97.97 & 7.447 & 0.553 & $\begin{array}{l}0.161 \\
0.166\end{array}$ & 0.030 & 0.00 & $\begin{array}{l}1.247 \\
1.399\end{array}$ & 0.5 & $\begin{array}{l}0.032 \\
0.034\end{array}$ & $\begin{array}{l}3.862 \\
2.863\end{array}$ & $\begin{array}{l}0.050 \\
0.050\end{array}$ & $\begin{array}{l}1.480 \\
1.763\end{array}$ & 0.187 & 0.000 & 0.006 & 15.173 & 0.68 & 0.006 \\
\hline 99.28 & 7.514 & 0.486 & 0.149 & 0.020 & 0.007 & 1.423 & 0.4 & 0.043 & $\begin{array}{l}2.803 \\
2.890\end{array}$ & 0.1 & $\begin{array}{l}1.672 \\
\end{array}$ & & 0.000 & 0.004 & 15.158 & 0.68 & 0.004 \\
\hline 98.84 & $\begin{array}{l}7.34 \\
7.809\end{array}$ & $\begin{array}{l}0.480 \\
0.191\end{array}$ & $\begin{array}{l}0.149 \\
0.102\end{array}$ & $\begin{array}{l}0.02017 \\
0.017\end{array}$ & $\begin{array}{l}0.017 \\
0.010\end{array}$ & $\begin{array}{l}1.243 \\
1.887\end{array}$ & $\begin{array}{l}0.468 \\
0.111\end{array}$ & $\begin{array}{l}0.043 \\
0.038\end{array}$ & $\begin{array}{l}2.890 \\
2.834\end{array}$ & $\begin{array}{l}0.146 \\
0.130\end{array}$ & $\begin{array}{l}1.1672 \\
1.803\end{array}$ & $\begin{array}{l}0.182 \\
0.067\end{array}$ & $\begin{array}{l}0.000 \\
0.000\end{array}$ & $\begin{array}{l}0.004 \\
0.000\end{array}$ & 15.036 & $\begin{array}{l}0.08 \\
0.61\end{array}$ & 0.000 \\
\hline 97.63 & 7.575 & 0.425 & 0.108 & 0.010 & 0.000 & $\begin{array}{l}1.081 \\
1.068\end{array}$ & 0.425 & 0.013 & 3.433 & 0.000 & 1.810 & 0.133 & 0.000 & 0.005 & 15.005 & 0.76 & 0.005 \\
\hline 97.06 & 7.327 & 0.673 & 0.268 & 0.035 & 0.000 & 1.000 & 0.551 & 0.028 & 3.145 & 0.000 & 1.760 & 0.214 & 0.000 & 0.000 & 15.000 & 0.76 & 0.000 \\
\hline
\end{tabular}

Notes: interst/cpx $=$ interstitial or replacing clinopyroxene; ground $=$ groundmass; plag crack $=$ filling crack in plagioclase .

Table 3. Distribution (\%) of amphibole compositions from different textural sites in Hole 504B dolerites, Legs 137/140.

\begin{tabular}{|c|c|c|c|c|c|c|}
\hline & Actinolite & $\begin{array}{c}\text { Fe- } \\
\text { Actinolite }\end{array}$ & $\begin{array}{c}\text { Act- } \\
\text { Hornblende }\end{array}$ & $\begin{array}{c}\text { Mg- } \\
\text { Hornblende }\end{array}$ & Edenite & $\begin{array}{l}\text { Cumming- } \\
\text { tonite }\end{array}$ \\
\hline Vein & 55 & 4.3 & 19 & 11 & 4.3 & \\
\hline Vein walls & 54 & 7.6 & 23 & 15 & & 4.3 \\
\hline Halo & 19 & 9 & 34 & 35 & & \\
\hline $\begin{array}{l}\text { Groundmass } \\
\text { (out of halo) }\end{array}$ & 36 & 5 & 29 & 15 & 12 & \\
\hline
\end{tabular}

\section{GEOTHERMOMETRY}

\section{Pyroxene}

The equilibrium crystallization temperature of hydrothermal clinopyroxene is constrained by the experimentally determined twopyroxene solvus (Lindsley, 1983; Lindsley and Anderson, 1983). These temperatures are strictly valid only when two pyroxenes coexist in equilibrium. Assuming that hydrothermal clinopyroxene grew in equilibrium with fluid and that there was no kinetic barrier to nucleating orthopyroxene if it was stable, the solvus temperature for a given clinopyroxene projected into the pyroxene quadrilateral (Lindsley and Anderson, 1983) represents a minimum equilibrium temperature. Thus, a composition that plots on the $500^{\circ} \mathrm{C}$ isotherm may have crystallized at higher temperature, but if it crystallized be-low $500^{\circ} \mathrm{C}$ the stable pyroxene would have been more calcic. The uncertainty in the solvus temperatures is about $\pm 20^{\circ}-110^{\circ} \mathrm{C}$ (typically $\pm 70^{\circ} \mathrm{C}$ ) for the hydrothermal pyroxenes from Hole 504B, which contain $2-18 \mathrm{~mol} \%$ (typically $10 \%$ ) non-quadrilateral components (uncertainty increases by about $\pm 5^{\circ} \mathrm{C}$ or each mol\% of non-quadrilateral components exceeding $2 \mathrm{~mol} \%$, according to Lindsley [1983]).

The temperatures of formation determined for the $\mathrm{Na}$, Ti-rich pyroxenes from Samples 137-504B-177R-1, 11-15 cm (Piece 4A) and 137-504B-180M-1, 74-78 cm (Piece 7), from 1606.2 to 1619.7 mbsf range from less than $500^{\circ}$ to $600^{\circ} \mathrm{C}$ (Fig. 10). The colorless secondary pyroxene (Samples 137-504B-173R-1,77-79 cm (Piece 9), 140-504B-189R-2, 100-104 cm (Piece 14), and 140-504B-213R$1,24-26 \mathrm{~cm}$ (Piece 7), from 1573.1 to $1812.8 \mathrm{mbsf}$ ) suggests minimum temperatures of equilibration ranging from $500^{\circ} \mathrm{C}$ to as high as about $700^{\circ} \mathrm{C}$ (Fig. 10). These temperatures are similar to those determined for secondary clinopyroxenes, some bearing aqueous fluid inclusions, from altered gabbros of Hole 735B in the Southwest Indian Ridge (Stakes et al., 1991; Vanko and Stakes, 1991).

The origin of the fluids associated with these secondary clinopyroxene is uncertain. $\mathrm{Na}$ and/or $\mathrm{Ti}$ and/or $\mathrm{Al}$-rich secondary augite has been reported previously from Hole 504B. It occurs sporadically in massive flows of the Upper Pillow Alteration Zone (in two samples at 572 mbsf depth: one in which $\mathrm{Ti}, \mathrm{Na}$-rich augite is associated with melanite, a Ti-rich garnet, and one containing aegirine augite

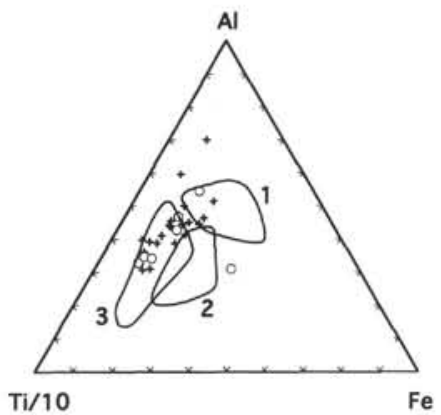

Figure 9. Al-Ti-Fe diagram for titanite from Hole 504B. Crosses: Legs 83/111; open circles: Legs 137/140. Reference composition fields are: 1 = prehnitepumpellyite facies, Central Sweden (Nyström, 1983); 2 = pumpellyite-actinolite facies, Central Sweden (Nyström, 1983); 3 = greenschist to amphibolite facies, East Taiwan ophiolite (Liou and Ernst, 1979).

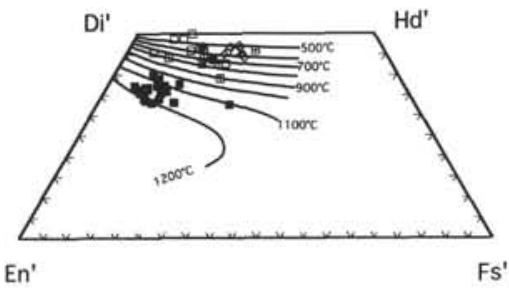

Figure 10. Clinopyroxene compositions, recalculated following the algorithm of Lindsley and Anderson (1983), plotted with isotherms of the 1-atm solvus (after Lindsley, 1983) for Hole 504B, Legs 137/140 samples. Di' is diopside component, $\mathrm{Hd}^{\prime}$ is hedenbergite, En' is enstatite, and Fs' is ferrosilite. Plot symbols are the same as for Figures 2, 3, and 4.

and Na-rich augite), in the Lower Pillow Alteration Zone (in three samples at 668 and 719 mbsf depth, one of which contains fassaite), in one sample from the transition zone between the pillow section and the sheeted dike complex, at 959 mbsf depth (Laverne, 1983; Laverne, 1987a), and in two samples from the dike complex cored during Leg 111, at 1436 and 1446 mbsf depth (Ishizuka, 1989). A deuteric origin was originally proposed for this secondary augite (Laverne, 1987a) because: (1) the process responsible for its formation does not depend on the depth; (2) this mineral occurs in each of the various zones characterized by very different alteration features (oxidative or reducing, high or low $\mathrm{T}$, high or low water/rock ratio, more or less evolved fluid); (3) it formed prior to any other alteration minerals; (4) this mineral is anhydrous, in contrast to hydrated minerals in Hole 504B, which are known to be hydrothermal in origin. 
Table 4. Representative plagioclase analyses from Hole 504B, Legs 137/140, keyed to spots on Plate 2A.

\begin{tabular}{|c|c|c|c|c|c|c|c|c|c|c|c|c|c|c|c|c|c|c|c|c|}
\hline $\begin{array}{l}\text { Core, section, } \\
\text { interval }(\mathrm{cm})\end{array}$ & Analysis & Type & $\mathrm{SiO}_{2}$ & $\mathrm{TiO}_{2}$ & $\mathrm{Al}_{2} \mathrm{O}_{3}$ & $\mathrm{FeO}$ & $\mathrm{MnO}$ & $\mathrm{MgO}$ & $\mathrm{CaO}$ & $\mathrm{SrO}$ & $\mathrm{Na}_{2} \mathrm{O}$ & $\mathrm{K}_{2} \mathrm{O}$ & $\mathrm{BaO}$ & $\mathrm{CuO}$ & Total & $\mathrm{Si}$ & $\mathrm{Ti}$ & $\mathrm{Al}$ & $\mathrm{Fe}$ & Mn \\
\hline \multirow[t]{6}{*}{$205 R-1.13-16$} & Al43 & Core & 49.63 & 0.06 & 31.29 & 0.52 & 0.00 & 0.26 & 15.27 & 0.17 & 2.92 & 0.00 & 0.00 & 0.00 & 100.12 & 2.271 & 0.002 & 1.688 & 0.020 & 0.000 \\
\hline & A145 & Core & 49.70 & 0.03 & 31.76 & 0.30 & 0.00 & 0.26 & 15.83 & 0.18 & 2.58 & 0.00 & 0.02 & 0.05 & 100.64 & 2.261 & 0.001 & 1.703 & 0.011 & 0.000 \\
\hline & Al44 & Rim & 46.19 & 0.00 & 34.46 & 0.12 & 0.00 & 0.01 & 17.92 & 0.11 & 1.28 & 0.00 & 0.03 & 0.03 & 100.09 & 2.124 & 0.000 & 1.868 & 0.005 & 0.000 \\
\hline & A 147 & $\operatorname{Rim}$ & 45.10 & 0.00 & 35.21 & 0.15 & 0.00 & 0.02 & 18.92 & 0.14 & 0.88 & 0.00 & 0.00 & 0.06 & 100.48 & 2.075 & 0.000 & 1.910 & 0.006 & 0.000 \\
\hline & A148 & Rim & 45.42 & 0.01 & 35.97 & 0.14 & 0.03 & 0.00 & 19.19 & 0.15 & 0.63 & 0.00 & 0.00 & 0.00 & 101.54 & 2.066 & 0.000 & 1.929 & 0.005 & 0.001 \\
\hline & Al46 & Veinlet & 66.10 & 0.02 & 22.20 & 0.20 & 0.00 & 0.00 & 2.29 & 0.20 & 10.43 & 0.02 & 0.04 & 0.00 & 101.50 & 2.868 & 0.001 & 1.136 & 0.007 & 0.000 \\
\hline
\end{tabular}

If we interpret all the secondary $\mathrm{Na}, \mathrm{Ti}$ diopside, and hedenbergite from Hole 504B as an early, high temperature, under low W/R ratio alteration from hydrothermal fluids derived from seawater, it means that such fluids were able to circulate everywhere in the hole, even in the pillow section. Why are these secondary clinopyroxene not more widespread?

We propose to interpret the green $\mathrm{Na}$, Ti-rich clinopyroxene as well as melanite and fassaite from the pillow section as resulting from an alteration by late magmatic fluids (deuteric alteration), possibly mixed with small volumes of hydrothermal fluids. The colorless diopside, without anomalous $\mathrm{Na}$ concentrations, recovered during Legs $137 / 140$ more probably has a hydrothermal origin.

\section{Amphibole/Plagioclase}

The more frequent occurrence of magnesio-hornblende (with increased $\mathrm{Al}$ and $\mathrm{Ti}$ concentrations) suggests higher maximum temperatures of alteration downhole (Raase, 1974; Laird and Albee, 1981). Independently, the first occurrence of secondary, hydrothermal calcic plagioclase suggests higher maximum temperatures as well. Recent experiments by Berndt and Seyfried (1993) have focused on the interaction of $\mathrm{Na}-\mathrm{Ca}-\mathrm{Cl}$ fluids and calcic plagioclase at $400^{\circ} \mathrm{C}$ and 400 bars. Results suggest that fluids with the chemical signature (particularly the $\mathrm{Ca}++/ \mathrm{Na}+$ ratio) of modern black smokers from the East Pacific Rise are in equilibrium at these conditions with labradorite. Thus, equilibrium with calcic plagioclase such as anorthite requires either higher temperature or higher $\mathrm{Ca}++/ \mathrm{Na}+$ in the fluid.

Fluid inclusions in calcic plagioclase generally exhibit homogenization temperatures in the range $150^{\circ}-160^{\circ} \mathrm{C}$, which, with an appropriate pressure correction, yield "trapping" temperatures of about $180^{\circ}-190^{\circ} \mathrm{C}$ (Kelley et al., this volume). This is not interpreted as the actual range of trapping temperatures, even though the inclusions appear to be primary. Instead, these are similar to the present-day borehole temperature, and suggest that inclusions in plagioclase continue to re-equilibrate along cleavage microcracks to the ambient temperature and pressure conditions.

Geothermometry based upon the equilibrium partitioning of $\mathrm{Na}$ and $\mathrm{Ca}$ between calcic amphibole and plagioclase may qualitatively help constrain alteration temperatures (Spear, 1980). In the Legs $137 / 140$ samples, amphiboles range from ferroactinolite or actinolite through actinolitic hornblende and magnesio-hornblende, and plagioclases vary from anorthite to albite in the same thin section. Petrographic evidence allows, however, that the earliest secondary plagioclase in vein halos, which is bytownite to anorthite, may have formed together with adjacent magnesio-hornblende in the same halos. According to the topology of the amphibole-plagioclase exchange, actinolitic hornblende and magnesio-hornblende can coexist with calcic labradorite through anorthite at temperatures exceeding $400^{\circ}-$ $500^{\circ} \mathrm{C}$ (Fig. 11).

\section{Chlorite and Chlorite-Smectite}

Chlorite-smectite exhibits several types of variation in the percentage of chlorite layers present. Chlorite-smectite in veins contains a greater proportion of chlorite layers than that replacing olivine in the host-rock of the same sample (Table 5), and chlorite-smectite in the rims of olivine pseudomorphs contains a greater percentage of chlorite layers than that at the cores of the pseudomorphs. In the intervals where relict olivine is present and where talc replaces olivine, chlorite-smectites contain relatively lower proportions of chlorite layers (1700-1820 mbsf, 1905-1938 mbsf, and 1980-1985 mbsf; Table 5 ; Fig. 8). Such variations can be explained by differences in temperature of reaction, or by variations in water-rock ratio and extent of reaction of the rocks, but in either case multiple stages of alteration are required.

Cathelineau and Nieva (1985) and Cathelineau (1988) have proposed a chlorite geothermometer based on variations in chlorite compositions with temperature in Los Azufres and other geothermal areas. Applying this geothermometer to the chlorite-smectites from Legs $137 / 140$ suggests a positive correlation between percent chlorite layers and increasing temperature (Fig. 12). This would imply higher temperatures of formation of chlorite-smectite in veins compared to host rocks, and higher temperatures during formation of chlorite-smectite replacing the rims of olivines than for that replacing the cores of olivine. The lower proportions of chlorite layers in chlorite-smectite in the zones where relict olivine is present and where talc replaces olivine also implies lower temperatures of formation of chlorite-smectite in these zones. Several problems exist with this geothermometer, however. Taking into account the errors involved in deriving the correlation for the geothermometer, the calculated temperatures can vary by $+50^{\circ} \mathrm{C}$. Moreover, chlorites from other geothermal areas do not all agree with the Los Azufres data (Cathelineau and Nieva, 1985). The data used to establish the "chlorite" geothermometer are actually analyses of chlorite-smectites: the presence of $\mathrm{Na}$ and $\mathrm{Ca}$ in the analyses were regarded as "contaminants" and discarded, when in actuality these indicate the presence of smectite layers and chlorite-smectite rather than chlorite (Shau and Peacor, 1992). Thus the chlorite geothermometer is really a chlorite-smectite geothermometer, and implies that chlorite-smectites are thermodynamic phases in equilibrium with rocks and fluids at the given temperatures, which is probably not the case for chlorite-smectite from Leg 140.

Even though the calculated range of temperature of chlorite and chlorite-smectite can be wide because of the various modes of occurrence within single samples, some significant variations with depth occur. Three zones of maximum higher temperature are clearly distinguished, at 1630-1650,1820, and 1980 mbsf. The 1630 and 1820 mbsf depths correspond to that where more Ti-rich and/or aluminous higher-temperature amphiboles occur.

An alternative explanation for variations in the percentage of chlorite layers in chlorite-smectite is that such variations are a function of reaction progress, reflecting the water/rock ratio and the kinetics of reaction of various phases in the rocks (Alt, 1984; Alt et al., 1985). Olivine is the first phase in the rocks to react with hydrothermal fluids. Thus, at very low water/rock ratios talc can form by simple hydration of olivine. This is borne out by the occurrence of relict olivine and talc replacing olivine in zones within the sheeted dikes where the rocks are only slightly altered and the abundance of veins is low from both core observations and logging measurements of the borehole walls (e.g., 1189-1319 mbsf, 1700-1820 mbsf, 1905-1938 mbsf, and 1980-1985 mbsf). At greater extents of reaction, plagio- 
Table 4 (continued).

\begin{tabular}{ccccccccc}
\hline $\mathrm{Mg}$ & $\mathrm{Ca}$ & $\mathrm{Sr}$ & $\mathrm{Na}$ & $\mathrm{K}$ & $\mathrm{Ba}$ & $\mathrm{Cu}$ & Total & $\%$ An \\
\hline 0.018 & 0.749 & 0.005 & 0.259 & 0.000 & 0.000 & 0.000 & 5.012 & 74.3 \\
0.018 & 0.772 & 0.005 & 0.228 & 0.000 & 0.000 & 0.002 & 5.000 & 77.2 \\
0.001 & 0.883 & 0.003 & 0.114 & 0.000 & 0.001 & 0.001 & 4.999 & 88.6 \\
0.001 & 0.933 & 0.004 & 0.079 & 0.000 & 0.000 & 0.002 & 5.009 & 92.2 \\
0.000 & 0.935 & 0.004 & 0.056 & 0.000 & 0.000 & 0.000 & 4.997 & 94.4 \\
0.000 & 0.106 & 0.005 & 0.878 & 0.001 & 0.001 & 0.000 & 5.003 & 10.8
\end{tabular}

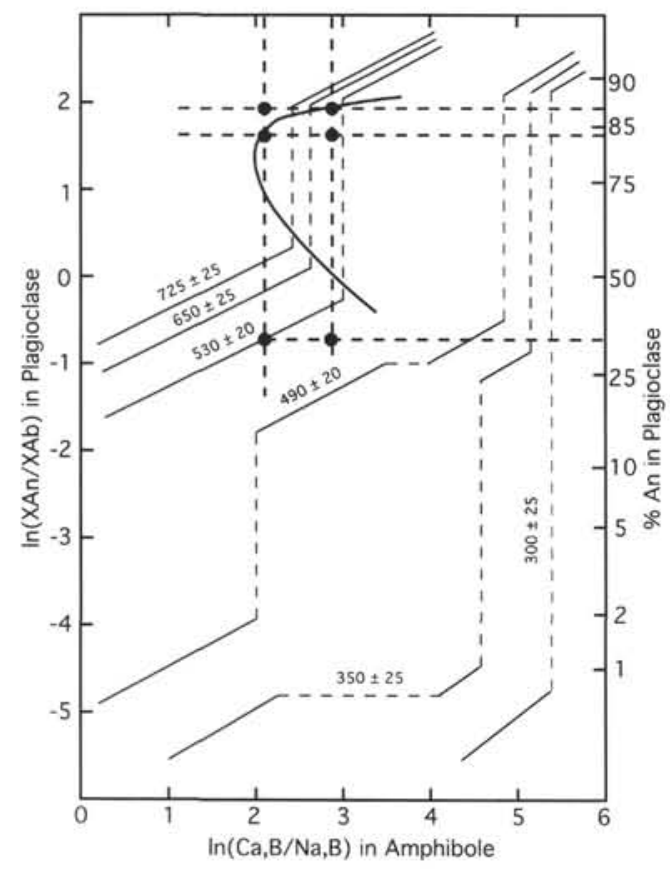

Figure 11. Topology of equilibrium between calcic amphibole and plagioclase, modified after Spear (1980). The ordinate corresponds to the plagioclase composition ( $\%$ anorthite component given along the right-hand edge). The abscissa value is calculated from the amphibole formula, where B represents the B-site (or M4 site). Generally, Hole 504B magnesio-hornblendes plot with a value of 1 to 3 , actinolites plot at values above 4 , and actinolitic hornblendes plot in between. The heavy curved line at the top depicts the calcic plagioclase solvus. Note that according to empirical temperature data from regionally metamorphosed rocks (temperatures labeled on diagram), the equilibrium coexistence of hornblende and anorthite-bytownite suggests temperatures exceeding $500^{\circ} \mathrm{C}$. Likewise, equilibrium coexistence of actinolitic-hornblende or actinolite with anorthite-bytownite suggests temperatures of $300^{\circ}-500^{\circ} \mathrm{C}$. Three electron microprobe analyses of plagioclase and two of hornblende from Sample 137-504B-176R-1 (Piece 3) are shown as heavy dashed lines. Their intersections are marked with filled circles. The actual location of these intersections is quite uncertain, however, because the value of the abscissa is poorly determined with electron microprobe analyses. Application of the constraints shown here are discussed more fully in the text.

clase is altered, releasing $\mathrm{Al}$, which is required to form chlorite. Thus, depending upon the extent of reaction and availability of $\mathrm{Al}$, various proportions of chlorite will form in chlorite-smectite. The occurrence of greater percentages of chlorite in chlorite-smectite in alteration halos around veins than in the host rock is consistent with this interpretation: water/rock ratios and the extent of reaction of the rocks were greater in the alteration halos than in the host rocks, farther from the solutions circulating along fractures. The influence of reaction progress on secondary mineralogy could also be recorded as variations in Fe content in the phyllosilicates due to variations in fluid composition (e.g., progressive alteration of titanomagnetite to sphene should release $\mathrm{Fe}$ ).

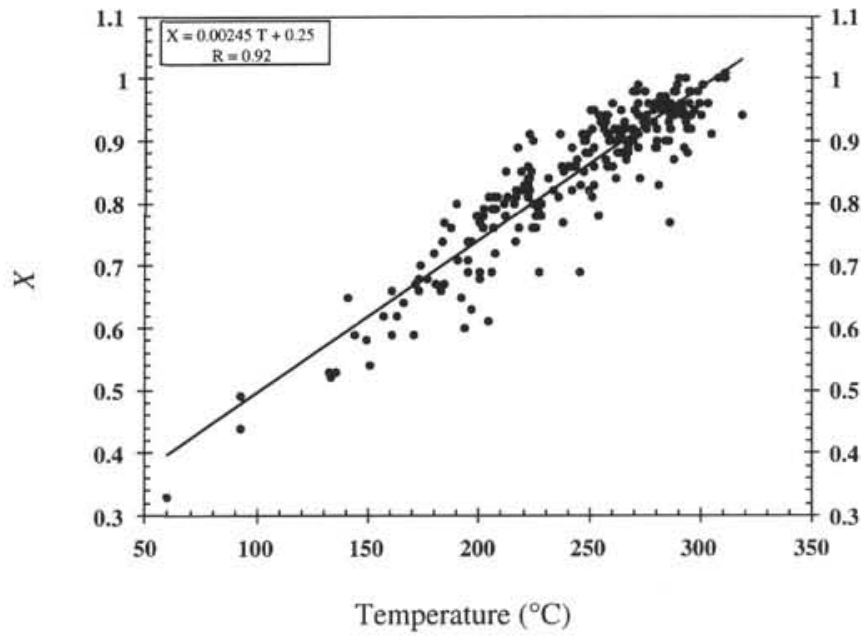

Figure 12. Plot of temperature (calculated using the Cathelineau geothermometer) vs. $X$ (the proportion of chlorite to swelling component; Bettison and Schiffman, 1988) for Legs $137 / 140$ chlorite and mixed-layer chlorite-smectite.

\section{DISCUSSION}

\section{Alteration Style}

Hydrothermal alteration is widespread, yet markedly heterogeneous, within the lower sheeted dike section sampled during Legs 137/140. On the scale of lithologic units sampled by the drill core (i.e., meter-scale and larger), variations in the intensity of hydrothermal alteration result in intervals that contain relict fresh olivine, fresh olivine mantled by talc alteration, or talc pseudomorphs of olivine. These intervals experienced restricted exposure to hydrothermal fluid such that the integrated water:rock ratio (W/R) must have been very much less than unity (down to zero according to $\delta^{18} \mathrm{O}$ data [Agrinier et al., this volume]). The total volume percent of alteration minerals in olivine, talc-bearing zones is low. Point count data recorded for 133 thin sections (Shipboard Scientific Party, 1992b) show that the average total percent alteration of 44 talc-bearing thin sections is $15 \mathrm{vol} \%$ (range 3\%-32\%), whereas the average for 89 talc-free thin sections is $34 \mathrm{vol} \%$ (range $0 \%-94 \%$ ).

On a finer scale, heterogeneous alteration is illustrated by the thin-section scale differences between vein halos and patches on the one hand and "background" altered dolerite on the other hand. Typically, halos and patches consist of greater than about 40 vol\% alteration phases, whereas the normal dolerite alteration varies from near zero (unusually) to about $40 \mathrm{vol} \%$. In the more highly altered background dolerite and in the halos/patches, olivine is completely altered, and chlorite and amphibole dominate the secondary phases. Talc is absent. This alteration heterogeneity at the $1-$ to $10-\mathrm{cm}$ scale reflects restricted access of hydrothermal fluids along fractures and other more tortuous permeability paths (e.g., interconnected zones of high primary porosity, perhaps due to gas vesicles, segregation vesicles, or diktytaxitic shrinkage void space). The $\delta^{18} \mathrm{O}$ values indicate a higher W/R ratio $(>0.7)$ in these permeable zones (Agrinier et al., this volume). Fluid mass transport into the host dolerite adjacent to these permeable features resulted in relatively restricted, centimeter-scale halos and patches. This mass transport was probably chiefly grain boundary diffusion, because a more substantial advective flow of hydrothermal fluid would have produced more pervasive heavy alteration. Hence, highly localized variations, on the scale of centimeters, in the proportions of secondary phases must reflect spatial and temporal variations in the extent of fluid-rock interaction, rather than temperature and/or pressure variations.

A final aspect of the heterogeneity of hydrothermal alteration is the lithologic control on alteration style. During the coring of steeply dipping sheeted dikes, dike margins and dike interiors were repeat- 
Table 5. Representative chlorite and chlorite-smectite analyses, Hole 504B, Legs 137/140.

\begin{tabular}{|c|c|c|c|c|c|c|c|}
\hline Core & & Expanded & Standard & & Temperature & & \\
\hline interval $(\mathrm{cm})$ & Analysis & depth & deviation & Occurrence & $\left({ }^{\circ} \mathrm{C}\right)$ & $\mathrm{x}$ & $\mathrm{SiO}_{2}$ \\
\hline $173 \mathrm{R}-1,77-79$ & B2174 & 1573.1 & 0.52 & ol & 264 & 0.95 & 28.58 \\
\hline & B2181 & 1573.1 & 0.52 & p & 290 & 1.00 & 27.65 \\
\hline $175 \mathrm{R}-1,1-5$ & B5126 & 1587.1 & 1.12 & of & 195 & 0.69 & 31.14 \\
\hline & $\begin{array}{l}\text { B5127 } \\
\text { B518 }\end{array}$ & 1587.1 & 1.12 & ol & 204 & 0.81 & 30.97 \\
\hline & B5128 & $\begin{array}{l}1587.1 \\
15964\end{array}$ & 1.12 & $\mathrm{pl}$ & 262 & 0.90 & 28.83 \\
\hline $176 \mathrm{R}-1,15-17$ & $\begin{array}{l}\text { A5 } \\
\text { A11 }\end{array}$ & $\begin{array}{l}1596.4 \\
1596.4\end{array}$ & $\begin{array}{l}0.51 \\
0.51\end{array}$ & $\begin{array}{l}v \\
y\end{array}$ & $\begin{array}{l}298 \\
292\end{array}$ & $\begin{array}{l}0.98 \\
0.95\end{array}$ & $\begin{array}{l}27.85 \\
28.02\end{array}$ \\
\hline $177 \mathrm{R}-1,11-15$ & B2106 & 1606.2 & 0.81 & $\mathrm{v}$ & 269 & 0.98 & 28.81 \\
\hline $180 \mathrm{M}-1,74-78$ & B5085 & 1619.7 & 0.20 & $\mathrm{p}$ & 200 & 0.77 & 30.99 \\
\hline & B5086 & 1619.7 & 0.20 & p & 246 & 0.83 & 28.60 \\
\hline $186 \mathrm{R}-1,140-143$ & B3106 & 1630.1 & 0.36 & p & 252 & 0.83 & 30.10 \\
\hline $186 \mathrm{R}-2,25-28$ & B3109 & 1630.1 & 0.36 & v & 216 & 0.80 & 30.66 \\
\hline $189 \mathrm{R}-1,105-106$ & $\begin{array}{l}\text { A37 } \\
\text { B2010 }\end{array}$ & $\begin{array}{l}16311.4 \\
1652.9\end{array}$ & $\begin{array}{l}0.25 \\
0.15\end{array}$ & $\underset{\mathrm{ol}}{\mathrm{p}}$ & $\begin{array}{l}311 \\
183\end{array}$ & $\begin{array}{l}1.00 \\
0.74\end{array}$ & $\begin{array}{l}27.60 \\
31.05\end{array}$ \\
\hline & B2012 & 1652.9 & 0.15 & ol & $\begin{array}{l}183 \\
180\end{array}$ & 0.72 & 31.01 \\
\hline $189 \mathrm{R}-1,106-110$ & V 5152 & 1653.0 & 0.15 & $\mathrm{v}$ & 301 & 0.99 & 27.28 \\
\hline $189 \mathrm{R}-2,11-13$ & B2024 & 1653.3 & 0.15 & cpx & 276 & 0.94 & 28.58 \\
\hline I89R-2, 76-79 & B2162 & 1654.0 & 0.15 & cpx & 291 & 0.96 & 27.83 \\
\hline & $\begin{array}{l}\mathrm{B} 2166 \\
\mathrm{~B} 3057\end{array}$ & 1654.0 & 0.15 & cpx & 279 & 0.95 & $\begin{array}{l}28.61 \\
3050\end{array}$ \\
\hline $\begin{array}{l}\text { 191R-1, 145-148 } \\
194 \mathrm{R}-1,59-61\end{array}$ & $\begin{array}{l}\text { B3057 } \\
\text { A55 }\end{array}$ & $\begin{array}{l}1670.5 \\
1684.6\end{array}$ & $\begin{array}{l}0.44 \\
0.80\end{array}$ & of & $\begin{array}{l}173 \\
305\end{array}$ & $\begin{array}{l}0.66 \\
0.91\end{array}$ & $\begin{array}{r}32.59 \\
27.75\end{array}$ \\
\hline $194 \mathrm{R}-1,84-87$ & $\begin{array}{l}\mathrm{A} 5 \mathrm{~S} 29 \\
\mathrm{~B} 029\end{array}$ & $\begin{array}{l}1084.6 \\
1686.7\end{array}$ & $\begin{array}{l}0.80 \\
0.76\end{array}$ & $\underset{o l}{p}$ & $\begin{array}{l}305 \\
93\end{array}$ & $\begin{array}{l}0.91 \\
0.44\end{array}$ & $\begin{array}{l}27.75 \\
35.42\end{array}$ \\
\hline & B5030 & 1686.7 & 0.76 & ol & 218 & 0.76 & 30.52 \\
\hline 195R-1, 7-8 & B5120 & & 1.32 & of & 228 & 0.78 & 29.86 \\
\hline & B5122 & 1696.5 & 1.32 & ol & 192 & 0.65 & 30.96 \\
\hline $196 \mathrm{R}-1,29-33$ & $\begin{array}{l}\text { B } 3081 \\
\mathrm{~B} 3002\end{array}$ & 1700.5 & 0.88 & ol & 228 & 0.80 & 30.41 \\
\hline $197 \mathrm{R}-1,44-46$ & $\begin{array}{l}\text { B33082 } \\
\text { B3075 }\end{array}$ & $\begin{array}{l}1700.5 \\
1704.9\end{array}$ & $\begin{array}{l}0.88 \\
0.53\end{array}$ & $\begin{array}{l}\text { of } \\
\text { ol.c }\end{array}$ & $\begin{array}{l}212 \\
190\end{array}$ & $\begin{array}{l}0.78 \\
0.71\end{array}$ & $\begin{array}{l}31.00 \\
31.31\end{array}$ \\
\hline & B3077 & 1704.9 & 0.53 & ol.r & 261 & 0.90 & $\begin{array}{l}31.31 \\
28.99\end{array}$ \\
\hline $200 \mathrm{R}-1,62-65$ & B4040 & 1729.8 & 0.20 & $\mathrm{v}$ & 284 & 0.97 & 28.34 \\
\hline $200 \mathrm{R}-3,81-84$ & B5037 & 1735.3 & 0.25 & ol & 261 & 0.92 & 29.37 \\
\hline & B5038 & 1735.3 & 0.25 & ol & 288 & 0.95 & 28.36 \\
\hline 200R-3, 108-112 & $\begin{array}{l}\text { V6174 } \\
\text { V6177 }\end{array}$ & $\begin{array}{l}1736.0 \\
17360\end{array}$ & 0.24 & ol.r. & ${ }_{105}^{223}$ & 0.84 & 29.82 \\
\hline $201 R-1,0-4$ & $\begin{array}{l}\text { V61177 } \\
83127\end{array}$ & $\begin{array}{l}1736.0 \\
7739.3\end{array}$ & $\begin{array}{l}0.24 \\
1.30\end{array}$ & cpx & $\begin{array}{l}195 \\
251\end{array}$ & 0.71 & $\begin{array}{l}30.92 \\
3014\end{array}$ \\
\hline & B4009 & $\begin{array}{l}1739.3 \\
1739.3\end{array}$ & $\begin{array}{l}1.30 \\
1.30\end{array}$ & $\begin{array}{l}\text { ol } \\
\text { ol }\end{array}$ & $\begin{array}{r}251 \\
60\end{array}$ & $\begin{array}{l}0.81 \\
0.33\end{array}$ & $\begin{array}{l}30.14 \\
36.16\end{array}$ \\
\hline & B4010 & 1739.3 & 1.30 & ol.r & 133 & 0.52 & 33.06 \\
\hline 202R-1, 53-55 & B5087 & 1748.6 & 0.11 & ol & 226 & 0.80 & 29.89 \\
\hline & B5088 & 1748.6 & 0.11 & ol & 161 & 0.59 & 32.17 \\
\hline $205 \mathrm{R}-1,6-9$ & $\begin{array}{l}\text { B5104 } \\
\text { B5105 }\end{array}$ & 1757.1 & 0.11 & $p$ & 247 & 0.85 & 29.29 \\
\hline & $\begin{array}{l}\text { B5505 } \\
\text { B5106 }\end{array}$ & $\begin{array}{l}1757.1 \\
1757.1\end{array}$ & $\begin{array}{l}0.11 \\
0.11\end{array}$ & $\stackrel{p}{v}$ & $\begin{array}{l}282 \\
285\end{array}$ & $\begin{array}{l}0.97 \\
0.96\end{array}$ & $\begin{array}{l}28.19 \\
27.78\end{array}$ \\
\hline & $\begin{array}{l}\mathrm{B} 5100 \\
\mathrm{~B} 5111\end{array}$ & $\begin{array}{l}7757.1 \\
1757.1\end{array}$ & 0.11 & ol & 224 & $\begin{array}{l}0.96 \\
0.76\end{array}$ & $\begin{array}{l}27.78 \\
30.47\end{array}$ \\
\hline $208 \mathrm{R}-2,76-81$ & B3103 & 1785.3 & 0.39 & ol.c & 141 & 0.65 & 3436 \\
\hline & B3104 & 1785.3 & 0.39 & ol.r & 243 & 0.06 & 3012 \\
\hline & B5009 & 1785.3 & 0.3 & ol & 206 & 079 & 3126 \\
\hline & B5014 & 1785.3 & $0.39>-2-1$ & ol. & 2022 & 0.86 & 30.56 \\
\hline & B5025 & 1785.3 & 0.39 & ol.c & 135 & 0.53 & 34.04 \\
\hline & B5026 & 1785.3 & 0.39 & ol.c & 173 & 0.68 & 32.32 \\
\hline $208 \mathrm{R}-3,1-4$ & A74 & 1787.5 & 0.13 & p & 262 & 0.88 & 28.85 \\
\hline & A78 & 1787.5 & 0.13 & $\mathrm{p}$ & 226 & 0.76 & 31.16 \\
\hline $209 \mathrm{R}-2,108-111$ & B 6022 & 1791.7 & 0.35 & p & 286 & 0.90 & 28.09 \\
\hline & B6026 & 1791.7 & 0.35 & p & 300 & 0.94 & 27.62 \\
\hline 209R-2, 146-149 & $\begin{array}{l}B 6522 \\
\text { V6178 }\end{array}$ & $\begin{array}{l}1791.7 \\
17947\end{array}$ & 0.35 & $p$ & 266 & 0.87 & 29.11 \\
\hline $209 \mathrm{k}=2,140-149$ & $\begin{array}{l}\text { V6178 } \\
\text { V6181 }\end{array}$ & $\begin{array}{l}1794.7 \\
1794.7\end{array}$ & 0.11 & ol $\begin{array}{c}\text { ol } \\
\text { ol }\end{array}$ & ${ }_{202}^{222}$ & $\begin{array}{l}0.81 \\
0.76\end{array}$ & 30.23 \\
\hline & B6013 & 1794.7 & 0.11 & v & ${ }_{209}^{292}$ & 0.96 & 2703 \\
\hline $210 \mathrm{R}-1,56-60$ & B3114 & 1795.9 & 0.16 & ol.c & 19 & 078 & 3120 \\
\hline & B3115 & 1795.9 & 0.16 & ol.c & 18 & 0.76 & 3141 \\
\hline 212R-1, 61-65 & B5095 & 1812.3 & $0.3-2>-1$ & ol out halo & ${ }_{21}^{16}$ & 08 & 2987 \\
\hline & B5096 & 1812.3 & $0.3>-3$ & ol out halo & 24 & 0.88 & 28,60 \\
\hline & B5097 & 1812.3 & 0.32 & ol in halo & 252 & 0.89 & 29.08 \\
\hline & B5098 & 1812.3 & 0.32 & ol in halo & 226 & 0.79 & 29.81 \\
\hline $214 \mathrm{R}-2,0-5$ & B3119 & 1820.9 & 0.14 & ol & 237 & 0.86 & 30.21 \\
\hline & B3121 & 1820.9 & 0.14 & ol & 259 & 0.90 & 29.75 \\
\hline $214 \mathrm{R}-2,29-32$ & P0037 & 1821.4 & 0. & $\mathrm{pl}$ & 2 & 0.96 & 27.86 \\
\hline $215 \mathrm{R}-1,43-46$ & $\begin{array}{l}\text { B5117 } \\
\text { B5118 }\end{array}$ & 1825.4 & 0.45 & p & 267 & 0.88 & 29.54 \\
\hline $216 \mathrm{R}-1.25-28$ & $\begin{array}{l}\text { B5118 } \\
\text { A83 }\end{array}$ & $\begin{array}{l}1825.4 \\
1831.3\end{array}$ & $\begin{array}{l}0.45 \\
0.96\end{array}$ & $p$ & $\begin{array}{l}295 \\
280\end{array}$ & 0.98 & $\begin{array}{r}27.61 \\
27.79\end{array}$ \\
\hline $216 \mathrm{R}-1,25-28$ & $\begin{array}{l}\mathrm{A} 83 \\
\mathrm{~A} 84\end{array}$ & $\begin{array}{l}1831.3 \\
1831.3\end{array}$ & $\begin{array}{l}0.96 \\
0.96\end{array}$ & $\begin{array}{l}\text { int } \\
\text { int }\end{array}$ & $\begin{array}{l}280 \\
261\end{array}$ & $\begin{array}{l}0.89 \\
0.84\end{array}$ & $\begin{array}{l}27.79 \\
29.12\end{array}$ \\
\hline $216 \mathrm{R}-1,58-60$ & & 1835.1 & 0. & $\mathrm{v}$ & 303 & 0.96 & 26.54 \\
\hline & B2035 & 1835.1 & 0.8 & ol & 257 & 0.94 & 28.91 \\
\hline $218 \mathrm{R}-120-23$ & B20 & 1835.1 & 0.88 & ol & 238 & 0.77 & 28.94 \\
\hline $218 \mathrm{R}-1,20-23$ & $\begin{array}{l}\text { B3043 } \\
\text { B3045 }\end{array}$ & $\begin{array}{l}1853.9 \\
18530\end{array}$ & 1.31 & $\mathrm{ol} / \mathrm{p}$ & 180 & 0.67 & 32.41 \\
\hline & $\begin{array}{l}\text { B3045 } \\
\text { B3046 }\end{array}$ & $\begin{array}{l}1853.9 \\
1853.9\end{array}$ & $\begin{array}{l}1.31 \\
1.31\end{array}$ & $\mathrm{v}$ & ${ }_{273}^{242}$ & $\begin{array}{l}0.82 \\
0.84\end{array}$ & $\begin{array}{l}29.37 \\
28.74\end{array}$ \\
\hline $222 \mathrm{R}-1,129-132$ & $\begin{array}{l}\text { B3346 } \\
\text { P0085 }\end{array}$ & $\begin{array}{l}18533.9 \\
1893.5\end{array}$ & $\begin{array}{l}1.31 \\
0.44\end{array}$ & ol & 263 & $\begin{array}{l}0.84 \\
0.88\end{array}$ & $\begin{array}{l}28.74 \\
29.01\end{array}$ \\
\hline & B3029 & 1896.8 & 1.18 & ol/p & 20 & 0.69 & 30.39 \\
\hline $224 R-1$ & & & $0.7>>>>$ & of & 26 & 0.93 & 28.17 \\
\hline & BS & 19 & 0 & of & 2 & 0 & 2880 \\
\hline $225 \mathrm{R}-1,126-129$ & & 19 & 0. & int & 25 & $0.8-2-2$ & 2880 \\
\hline & & & & int & 2 & & 30.70 \\
\hline & & 191 & & cpx & 144 & & 34.09 \\
\hline 226R-1,99-102 & B2 & & 0. & ol/int & 254 & 0.94 & 29.62 \\
\hline & & & 0. & ol & 2 & 0.92 & 29.59 \\
\hline & B2045 & 1921.4 & 0. & ol & 161 & 0.66 & 33.30 \\
\hline $226 \mathrm{R}-3,5-9$ & $\begin{array}{l}\text { B2077 } \\
\text { B2007 }\end{array}$ & $\begin{array}{l}1924.0 \\
19240\end{array}$ & 0.07 & $p$ & $\begin{array}{l}237 \\
269\end{array}$ & 0.91 & 29.77 \\
\hline & & & & & & & \\
\hline $228 \mathrm{R}-1,58-60$ & B & 19. & & ol.c & & 0.49 & 35.51 \\
\hline & & & & & & & \\
\hline $236 \mathrm{R}-1,0-4$ & & & 0 & ol. $r$ & 293 & i. 2 & 28.45 \\
\hline & & & & ol & 157 & 0.62 & 33.17 \\
\hline & & 19 & & ol.c & 132 & 0.53 & 33.72 \\
\hline & & 1980.9 & 0.14 & ol.r & 272 & 0.96 & 28.41 \\
\hline $236 \mathrm{R}-1,30-32$ & $\begin{array}{l}\text { B3136 } \\
B_{3} 1337\end{array}$ & 1981.8 & 0.27 & ol & 149 & 0.58 & 32.93 \\
\hline 236R-1,57-60 & $\begin{array}{l}\text { B31137 } \\
\text { B3087 }\end{array}$ & 19 & $\begin{array}{l}0 . \\
0 .\end{array}$ & $\begin{array}{l}\text { ol } \\
\text { ol }\end{array}$ & 29 & 0.94 & $\begin{array}{l}31.72 \\
28.53\end{array}$ \\
\hline $237 \mathrm{R}-1,24-26$ & B3009 & 1991.1 & 0.90 & ol & 183 & 0.66 & 31.93 \\
\hline & B3012 & 1991.1 & 0.90 & ol & 223 & 0.82 & 30.13 \\
\hline
\end{tabular}

Notes: ol = olivine replacement; ol. $\mathrm{c}=$ olivine core replacement; ol. $\mathrm{r}=$ olivine rim replacement; $\mathrm{pl}=$ plagioclase replacement; $\mathrm{cpx}=$ clinopyroxene replacement; $\mathrm{v}=\mathrm{vein} ; \mathrm{p}=$ patch: int $=$ interstitial. 
Table 5 (continued).

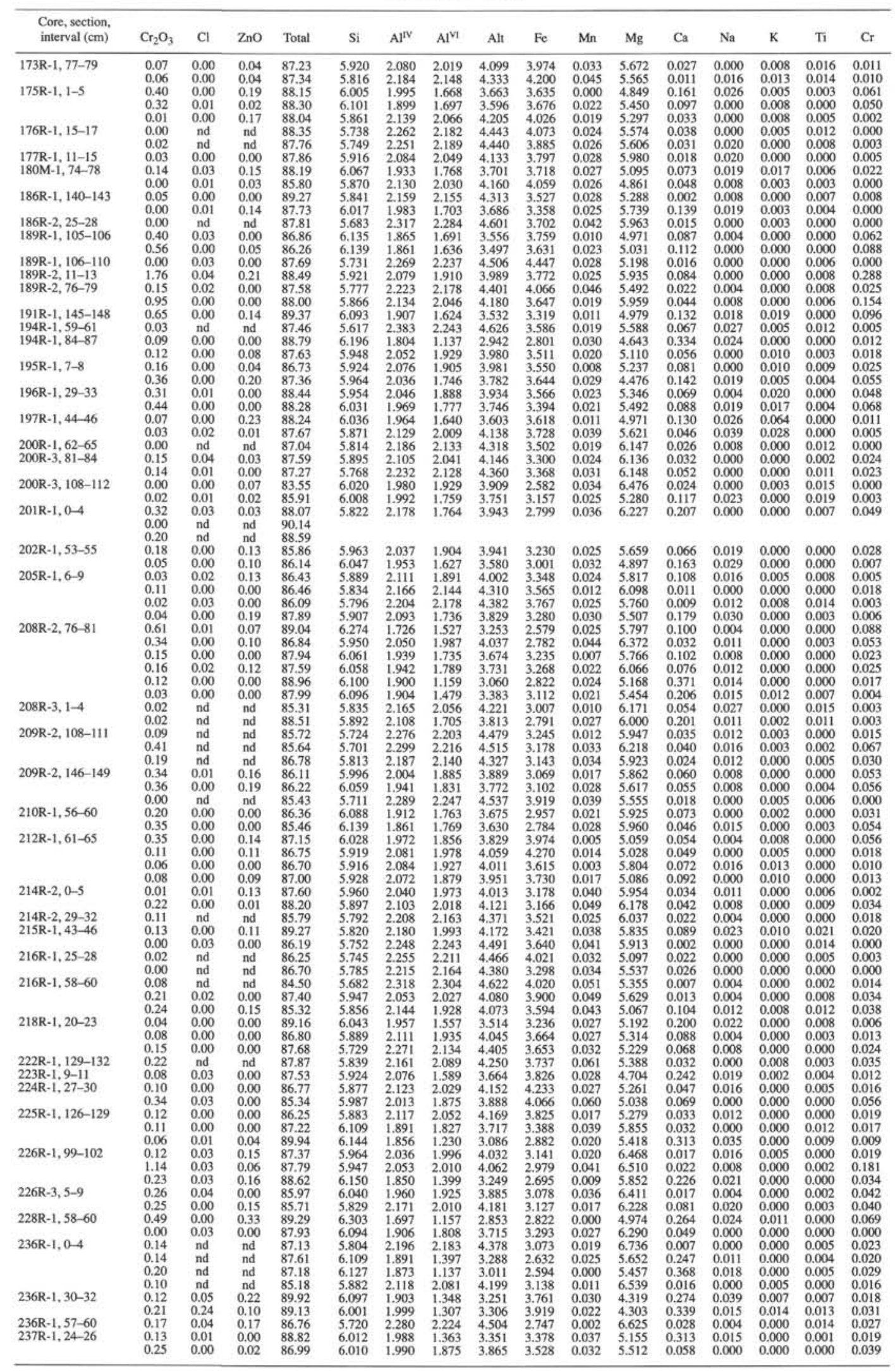


edly encountered. Fracture intensity is demonstrably higher in the finer-grained dike-margin rocks, presumably because of the higher thermal contractional stresses experienced by aphanitic and finegrained dike margins relative to medium-grained dike interiors. Thus, vein density and, by inference, the integrated $W / R$ ratio are also higher in dike margin rocks. Significantly, the few veins bearing quartz and/or epidote occur in association with fine-grained rocks and aphanitic chilled margins.

Evidence from cross-cutting veins and mineral pseudomorphs indicates that many samples apparently underwent several episodes of hydrothermal alteration (Shipboard Scientific Party, 1992a, b). This could be due to the episodic nature of local heat sources in a dike swarm: each individual dike intrusion, or each episode of swarm intrusion, is capable of reestablishing a new hydrothermal system that cools the newly intruded material. Such episodicity means that "early" high-temperature minerals may postdate low-temperature minerals from a previous hydrothermal alteration. Nevertheless, for simplicity we may place the observed alteration minerals in a sequence of formation that begins at high temperature and continues to lower temperature with time. This sequence is frequently confirmed by geometrical evidences.

The earliest alteration, related to early fracture-controlled introduction of fluid (probably mixed deuteric and hydrothermal fluids) resulted in the restricted development of secondary $\mathrm{Na}$ - and Ti-rich diopside at temperatures between about $500^{\circ} \mathrm{C}$ and $600^{\circ} \mathrm{C}$. This alteration is closely followed by the development of vein halos that contain magnesio-hornblende and secondary anorthite or bytownite plagioclase, with estimated temperatures exceeding $400^{\circ}-500^{\circ} \mathrm{C}$. Very small amounts of secondary diopside without anomalous $\mathrm{Na}$ or $\mathrm{Ti}$ occur locally in veinlets and patches and possibly formed prior to hornblende and anorthite, suggesting a water/rock ratio low and then higher.

The second alteration stage is characterized by the growth of actinolite and actinolitic hornblende with chlorite in veins; albite forming veinlets that crosscut primary igneous plagioclase as well as secondary calcic plagioclase; and chlorite, chlorite-smectite, or talc after olivine in lower permeability rocks. This stage is generally lower temperature, estimated at around $250^{\circ}-320^{\circ} \mathrm{C}$.

The third stage is represented by epidote and quartz in veins and vugs and minor epidote after host-rock plagioclase. Oxygen isotope analyses of two pairs of epidote and quartz from veins of the Legs $137 / 140$ section indicate temperatures of $310^{\circ}-320^{\circ} \mathrm{C}$ for these minerals (Alt et al., this volume) and are similar to temperatures estimated from quartz and epidote from the upper dikes and transition zone (Alt et al., 1986b).

During a fourth stage, anhydrite crystallized, probably directly from heated seawater.

Finally, laumontite and prehnite formed at temperatures probably less than or equal to about $250^{\circ} \mathrm{C}$.

\section{Origin of Hydrothermal Fluid}

Hydrothermal fluids trapped in fluid inclusions in secondary plagioclase and, rarely, in quartz, represent aqueous solutions with seawaterlike salinities (Kelley et al., this volume). Many inclusions bear dissolved gas (possibly $\mathrm{CO}_{2}$ or $\mathrm{CH}_{4}$ ), though at concentrations and pressures too low to form a liquid gas phase at room temperature. The most likely origin of such fluids is a hydrothermal, modified seawater with an unknown, most probably very small magmatic contribution.

Independent evidence that the hydrothermal fluid was seawater is the stable isotopic signature of altered rocks (Agrinier et al., this volume). Oxygen isotopic whole-rock values are typically shifted down from the primary magmatic value of $+5.6 \%$, resulting from the hightemperature $\left(>250^{\circ} \mathrm{C}\right)$ oxygen isotopic exchange with hydrothermal seawater (which is probably about $0 \%$ to $+2 \%$; e.g., Stakes and O'Neill, 1982)

Chlorine concentrations in amphiboles are typical for seawateraltered, amphibole-bearing deep crustal rocks from the ocean, wherein actinolite has minor $\mathrm{Cl}$ (less than $0.05 \mathrm{wt} \% \mathrm{Cl}$ ), hornblendes typically have 0.1 to $1.5 \mathrm{wt} \% \mathrm{Cl}$, and rare $\mathrm{Cl}$-rich hornblendes contain up to about 4.0 wt \% Cl (e.g., Vanko, 1986; Vanko and Stakes, 1991). In the Legs $137 / 140$ rocks, actinolite is $\mathrm{Cl}$-poor, and hornblende contains up to about $0.5 \mathrm{wt} \% \mathrm{Cl}$. The lack of $\mathrm{Cl}$-rich amphibole may indicate that unusually briney hydrothermal fluids did not develop in the lower sheeted dikes during water-rock interaction, at least in this area.

The mere volume of hydrothermal minerals observed in the sheeted dikes of Hole 504B may require a seawater source of the fluid as opposed to a magmatic source. A typical estimate of the total volume of alteration minerals based on thin-section point counts (Shipboard Scientific Party, 1992b) is about $30 \mathrm{vol} \%$. If these minerals are approximated by amphibole ( $2 \mathrm{wt} \%$ water) and chlorite (12 wt \% water) in a 5:1 ratio, the dolerite contains an estimated $1.1 \mathrm{wt} \%$ water, which exceeds most estimates of the water content of typical primitive midocean-ridge basalt.

\section{Implications for Crustal Alteration}

Several results of the present study are significant in contributing to our understanding of the alteration of oceanic crust. First, all of Hole 504B has undergone hydrothermal alteration, and the present results extend those of previous studies that described the low-temperature oxidative alteration of the upper pillowed section, the low-temperature reducing alteration of the lower pillows, and the greenschist-like alteration of the transition zone and the sheeted dikes. The lower sheeted dikes of Legs 137/140 experienced higher temperature alteration in general, as evidenced by more prevalent actinolitic hornblende and, especially, abundant magnesio-hornblendes.

With the exception of the stockwork-like alteration in the transition zone (Honnorez et al., 1985) and minor quartz-epidote assemblages along dike margins, practically all of the sheeted dike alteration results from non-focused, pervasive fluid-rock interaction as opposed to focused, high-temperature, high W/R discharge alteration. In the terminology of "discharge" and "recharge" alteration as described by Harper et al. (1988) for the Josephine ophiolite, the Hole 504B dike alteration is probably within the "recharge," or even within a "reaction zone" milieu. The characteristics of this reaction zone, then, include an initial high-temperature development of secondary clinopyroxene, followed closely by a calcic plagioclase-hornblende assemblage (amphibolite facies), then a pervasive greenschist-like actinolite-chloritesodic plagioclase assemblage. Finally, interaction with cooler fluids caused the crystallization of zeolites, prehnite, and anhydrite.

The alteration style described here for Hole 504B differs in significant ways from alteration described from ophiolite sections and from other oceanic sample suites. One major new discovery in Hole $504 \mathrm{~B}$ is the presence of hydrothermal anorthite-bytownite, in association with magnesio-hornblende in amphibole vein halos. This assemblage may partly verify the recent prediction (Berndt and Seyfried, 1993 ) that the plagioclase that should be found in the reaction zone for modern black smoker fluids is calcic labradorite. This prediction is based upon experiments reacting plagioclase and $\mathrm{Na}-\mathrm{Ca}-\mathrm{Cl}$ fluids, and is understood as a consequence of (1) the high temperatures inferred for the reaction zone, such that sodic plagioclases are not stable, and (2) the fact that reacted seawater, having already affected the formation of secondary albite at lower temperature, is enriched in calcium by the time the reaction zone is reached. The discovery of secondary anorthite-bytownite is an extension of recent descriptions of intermediate secondary plagioclase in other oceanic sample suites (e.g., oligoclase and andesine in Hole 735B gabbros [Vanko and Stakes, 1991]), and oligoclase in Oceanographer Transform gabbros [Vanko et al., 1992]).

\section{CONCLUSIONS}

1. Hydrothermal alteration is widespread, but heterogeneous, in the studied dike section. This heterogeneity, which is related to the 
Table 6. Representative talc and mixed-layer talc-chlorite analyses, Hole 504B, Legs 137/140.

\begin{tabular}{|c|c|c|c|c|c|c|c|c|c|c|c|c|c|c|c|c|c|c|c|c|c|c|c|c|c|c|c|}
\hline $\begin{array}{l}\text { Core, section, } \\
\text { interval }(\mathrm{cm})\end{array}$ & Analysis & $\mathrm{SiO}_{2}$ & $\mathrm{Al}_{2} \mathrm{O}_{3}$ & $\mathrm{FeO}$ & $\mathrm{MnO}$ & $\mathrm{MgO}$ & $\mathrm{CaO}$ & $\mathrm{Na}_{2} \mathrm{O}$ & $\mathrm{K}_{2} \mathrm{O}$ & $\mathrm{TiO}_{2}$ & $\mathrm{Cr}_{2} \mathrm{O}_{3}$ & Total & $\mathrm{Si}$ & $\mathrm{Al}$ & $\mathrm{Al}^{\mathrm{IV}}$ & $\mathrm{Al}^{\mathrm{Vl}}$ & $\mathrm{Fe}$ & $\mathrm{Mn}$ & $\mathrm{Mg}$ & $\mathrm{Ca}$ & $\mathrm{Na}$ & $\mathrm{K}$ & $\mathrm{Ti}$ & $\mathrm{Cr}$ & Total & IV & V1 \\
\hline $175 \mathrm{R}$ & B5125 & 5 & 0.22 & 2.03 & 0.00 & 29.64 & 0.06 & 0.00 & & 0.02 & 0.01 & & 8 & 3 & 0 & & 8 & 0.000 & & 8 & 0 & 3 & 22 & 01 & 38 & 8.028 & 5.926 \\
\hline \multirow{6}{*}{$200 \mathrm{R}-1,62-65$} & & & & & & & & & & & & & & & & & & & & & & & & & & & \\
\hline & B & 6 & 63 & 2. & 0. & 29. & 0. & & 0. & & 0. & 94 & $\begin{array}{l}1 \\
1 \\
1\end{array}$ & 0.096 & & 0.000 & 0.284 & & 5.7 & 5 & & 0 & 0 & ${ }_{0}^{\infty}$ & & 7.997 & 61 \\
\hline & & & 94 & & & 8 & 0.74 & & & & 0. & & & 0306 & & & & & & & & & & & & 8.000 & \\
\hline & & & 92 & & & 22.97 & 33 & & & & 0. & & & 0.64 & 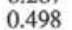 & & & & & & & & & & & & \\
\hline & & & & & & & & & & & & & & 0.222 & 0. & & 0.7 & 0.000 & & 34 & & & 0 & & & & \\
\hline & & 6 & 0.21 & 2. & & 29.14 & 0.11 & & & & 0.07 & & & 0.033 & & 00 & & & & & & & & & & & \\
\hline \multirow{2}{*}{ 200R-2, 18-24 } & & 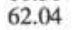 & & & & 28 & 0.03 & & & & 0. & & & 0.049 & 0.0 & 0.037 & 0.4 & 0.0 & 5.4 & 0.004 & 0.017 & 0.000 & 0.000 & 0 & & 8.000 & 5.996 \\
\hline & $\mathrm{V}$ & & 0.42 & & & 28. & 0. & & & & & & 2 & 0.065 & 0.0 & 0.0 & 0.4 & 0.0 & 5.5 & 0.0 & 0.028 & 0.000 & 0.000 & 0.003 & & 7.997 & 6.051 \\
\hline \multirow[t]{3}{*}{ 200R-2, 101-104 } & R & & & & & & & & & & & & & 0. & 0. & & $0.7 \quad-7$ & & & 0.0 & & & 0.0 & & & 8.000 & 6.071 \\
\hline & & & & & & & & & & & & & & 0.1 & & & 0.6 & & 5.1 & 0.0 & & & 0.000 & & & & 5.979 \\
\hline & & & & & & 27 & & & & & & & 3 & 0.104 & & & 0.6 & & 5.4 & 0.050 & & & 0.0 & & & 7.967 & 6.132 \\
\hline \multirow[t]{2}{*}{$200 \mathrm{R}-3,81-84$} & & & & & & 29. & & & & & & & & 0.070 & & & 0.200 & & 5.747 & 0.0 & 0. & & 0.000 & 0.014 & & 8.000 & 6.010 \\
\hline & $\mathrm{B}$ & 61.87 & 0.32 & 2.36 & & 29 & 0. & & & & & & & 9 & & 0. & 0.256 & 0. & 5.6 & 0. & 3 & 0 & 0 & 0 . & & 2 & 5.937 \\
\hline \multirow{3}{*}{$210 \mathrm{R}-1,56-60$} & & 62.72 & 0.13 & 2.07 & 0 & 30. & 0. & & & & & & & 0.0 & & 0. & 0.2 & 0 . & 5.7 & 0.0 & & & 0.0 & 0.007 & & 8.001 & 992 \\
\hline & B311 & 61.41 & 13 & 2. & 0 & 30. & 0. & 0. & 0. & & & & & 0.0 & 0. & & 0.282 & & & 0.0 & & & 0.0 & 0 . & & 7.971 & 6.105 \\
\hline & & & & 3.0 & & 30 & & & & & & & & & & & & & & & & & & 0 & & 7.966 & 6.165 \\
\hline & & 60.84 & 22 & 1.5 & 00 & .02 & 07 & 03 & 00 & 0. & c & 2.5 & 7.973 & 05 & 02 & 00 & .174 & .00 & 785 & .018 & .00 & .000 & .003 & 0.017 & 4.03 & 8.000 & 6.003 \\
\hline
\end{tabular}


Table 7. Representative epidote analyses, Hole 504B, Legs 137/140.

\begin{tabular}{llllllllllllll}
\hline $\begin{array}{c}\text { Core, section, } \\
\text { interval (cm) }\end{array}$ & Analysis & $\mathrm{SiO}_{2}$ & $\mathrm{Al}_{2} \mathrm{O}_{3}$ & $\mathrm{Fe}_{2} \mathrm{O}_{3}$ & $\mathrm{MnO}$ & $\mathrm{MgO}$ & $\mathrm{CaO}$ & $\mathrm{Na}_{2} \mathrm{O}$ & $\mathrm{K}_{2} \mathrm{O}$ & $\mathrm{TiO}_{2}$ & $\mathrm{Cr}_{2} \mathrm{O}_{3}$ & $\mathrm{Cl}$ & Total \\
\hline $193 \mathrm{R}-1,44-46$ & $\mathrm{P} 44$ & 37.46 & 23.51 & 10.98 & 0.00 & 0.10 & 23.16 & 0.03 & 0.00 & 0.07 & 0.00 & nd & 95.31 \\
$209 \mathrm{R}-2,108-111$ & $\mathrm{~B} 6043$ & 38.51 & 23.24 & 12.75 & 0.18 & 0.20 & 23.31 & 0.02 & 0.00 & 0.01 & 0.00 & nd & 98.22 \\
& $\mathrm{~B} 6045$ & 38.08 & 22.52 & 13.49 & 0.09 & 0.20 & 23.31 & 0.00 & 0.01 & 0.00 & 0.06 & nd & 97.76 \\
$213 \mathrm{R}-1,45-47$ & $\mathrm{P} 65$ & 38.13 & 21.82 & 12.70 & 0.08 & 0.88 & 21.76 & 0.03 & 0.00 & 0.10 & 0.00 & nd & 95.42 \\
& $\mathrm{P} 66$ & 37.01 & 23.43 & 11.95 & 0.03 & 0.17 & 22.65 & 0.01 & 0.02 & 0.06 & 0.00 & nd & 95.30 \\
$214 \mathrm{R}-1,67-70$ & $\mathrm{~B} 2193$ & 39.53 & 21.75 & 12.18 & 0.00 & 0.53 & 23.09 & 0.09 & 0.09 & 0.04 & 0.00 & 0.02 & 97.32 \\
& $\mathrm{~B} 2194$ & 38.54 & 22.97 & 14.20 & 0.05 & 0.07 & 23.59 & 0.06 & 0.01 & 0.19 & 0.00 & 0.02 & 99.65 \\
$214 \mathrm{R}-1,76-78$ & $\mathrm{P} 3$ & 337.63 & 24.41 & 11.71 & 0.14 & 0.09 & 23.77 & 0.00 & 0.00 & 0.06 & 0.02 & nd & 97.69 \\
& $\mathrm{P} 5$ & 37.35 & 24.09 & 11.94 & 0.05 & 0.04 & 23.49 & 0.00 & 0.00 & 0.16 & 0.01 & nd & 97.08 \\
$216 \mathrm{R}-1,7-10$ & $\mathrm{~A} 13$ & 38.57 & 26.48 & 8.76 & 0.17 & 0.02 & 23.65 & 0.03 & 0.01 & 0.05 & nd & nd & 97.74 \\
$216 \mathrm{R}-1,13-16$ & $\mathrm{~A} 15$ & 37.69 & 21.17 & 14.30 & 0.10 & 0.00 & 23.45 & 0.00 & 0.02 & 0.22 & nd & nd & 96.95 \\
& $\mathrm{~A} 16$ & 37.91 & 23.81 & 10.59 & 0.11 & 0.04 & 23.76 & 0.00 & 0.00 & 0.07 & nd & nd & 96.29 \\
$216 \mathrm{R}-1,58-60$ & $\mathrm{~B} 2033$ & 35.66 & 22.51 & 11.21 & 0.15 & 0.27 & 21.87 & 0.00 & 0.00 & 0.00 & 0.04 & 0.03 & 91.59 \\
& $\mathrm{~B} 2034$ & 36.59 & 24.02 & 9.52 & 0.28 & 0.01 & 21.91 & 0.00 & 0.01 & 0.03 & 0.00 & 0.00 & 92.09 \\
$217 \mathrm{R}-1,0-2$ & $\mathrm{P} 77$ & 38.23 & 25.55 & 10.43 & 0.15 & 0.02 & 23.40 & 0.02 & 0.00 & 0.13 & 0.00 & nd & 97.78 \\
& $\mathrm{P} 79$ & 377.70 & 26.35 & 10.23 & 0.17 & 0.02 & 23.58 & 0.00 & 0.00 & 0.13 & 0.00 & nd & 98.01 \\
$226 \mathrm{R}-3,5-9$ & $\mathrm{~B} 2075$ & 39.99 & 21.30 & 12.51 & 0.04 & 1.96 & 22.20 & 0.03 & 0.00 & 0.09 & 0.00 & 0.00 & 98.08 \\
& $\mathrm{~B} 2076$ & 38.55 & 23.59 & 11.94 & 0.15 & 0.29 & 23.27 & 0.00 & 0.02 & 0.11 & 0.08 & 0.00 & 97.85 \\
\hline
\end{tabular}

Note: Structural formulas are calculated on the basis of 12.5 oxygens.

Table 8. Representative prehnite analyses, Hole 504B, Legs 137/140.

\begin{tabular}{lllllllllllll}
\hline $\begin{array}{c}\text { Core, section, } \\
\text { interval (cm) }\end{array}$ & Analysis & $\mathrm{SiO}_{2}$ & $\mathrm{Al}_{2} \mathrm{O}_{3}$ & $\mathrm{Fe}_{2} \mathrm{O}_{3}$ & $\mathrm{MnO}$ & $\mathrm{MgO}$ & $\mathrm{CaO}$ & $\mathrm{Na}_{2} \mathrm{O}$ & $\mathrm{K}_{2} \mathrm{O}$ & $\mathrm{TiO}_{2}$ & $\mathrm{Cr}_{2} \mathrm{O}_{3}$ & $\mathrm{Total}$ \\
\hline $214 \mathrm{R}-2,29-32$ & $\mathrm{P} 25$ & 42.55 & 20.75 & 3.19 & 0.00 & 0.60 & 24.66 & 0.05 & 0.25 & 0.01 & 0.05 & 92.11 \\
& $\mathrm{P} 27$ & 42.56 & 20.80 & 3.06 & 0.06 & 0.24 & 25.57 & 0.04 & 0.00 & 0.05 & 0.00 & 92.38 \\
$216 \mathrm{R}-1,7-10$ & $\mathrm{~A} 81$ & 43.89 & 24.32 & 0.04 & 0.03 & 0.00 & 27.02 & 0.02 & 0.00 & 0.00 & nd & 95.32 \\
& $\mathrm{~A} 82$ & 43.51 & 23.58 & 0.06 & 0.00 & 0.00 & 24.91 & 0.08 & 0.00 & 0.00 & nd & 92.14 \\
$216 \mathrm{R}-1,13-15$ & $\mathrm{~A} 85$ & 42.60 & 23.43 & 1.98 & 0.03 & 1.13 & 23.42 & 0.00 & 0.01 & 0.02 & nd & 92.62 \\
& $\mathrm{~A} 86$ & 42.49 & 23.65 & 2.69 & 0.04 & 1.15 & 24.88 & 0.00 & 0.00 & 0.01 & nd & 94.91 \\
$216 \mathrm{R}-1,58-60$ & $\mathrm{P} 55$ & 42.65 & 23.58 & 1.96 & 0.24 & 0.77 & 25.06 & 0.00 & 0.01 & 0.00 & 0.03 & 94.30 \\
& $\mathrm{P} 56$ & 41.89 & 22.81 & 3.13 & 0.14 & 1.34 & 24.36 & 0.03 & 0.02 & 0.03 & 0.02 & 93.77 \\
$222 \mathrm{R}-1,129-132$ & $\mathrm{P} 82$ & 41.87 & 22.53 & 2.53 & 0.06 & 1.77 & 23.93 & 0.04 & 0.00 & 0.00 & 0.00 & 92.73 \\
\hline
\end{tabular}

Note: Structural formulas are calculated on the basis of 11 oxygens.

Table 9. Representative laumonite and heulandite analyses, Hole 504B, Legs 137/140.

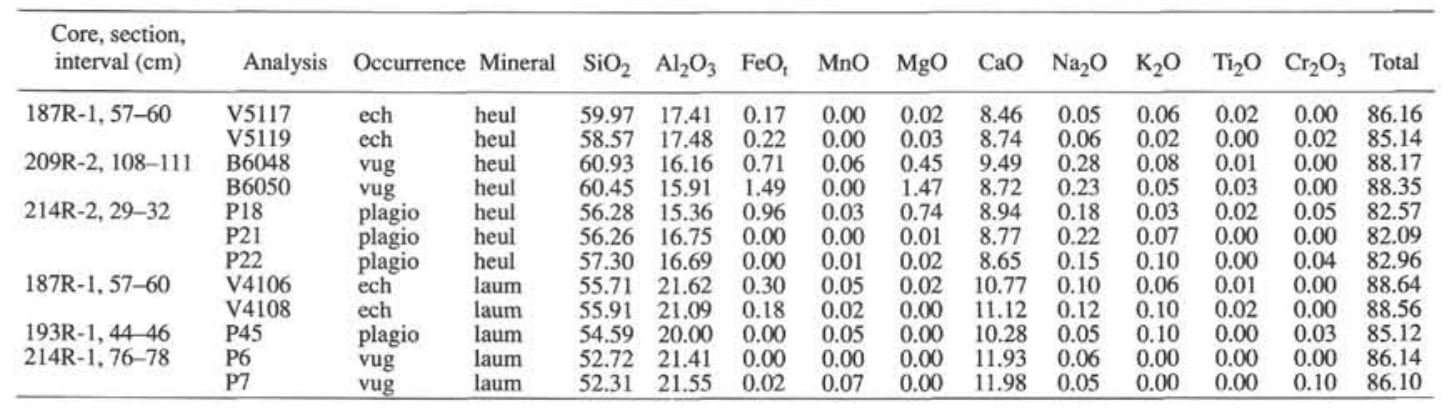

Notes: laum = laumonite; heul = heulandite; $e c h=$ "échelon" crack-filling; plagio = partial plagioclase replacement; vug = vug composing the internal part of some alteration patches. Structural formulas are calculated on the basis of 72 oxygens for heulandite and 48 oxygens for laumonite.

permeability of the oceanic crust, is observed at various scales: at meter-scale, with intervals of poorly altered dolerites containing fresh olivine and interpreted as having experienced restricted exposure to hydrothermal fluids; at centimeter- or millimeter-scale, with alteration patches and extensively altered halos adjacent to veins.

2. One important alteration feature of Legs $137 / 140$ is the formation of secondary clinopyroxene, before other secondary minerals. Two types of secondary clinopyroxene occur sporadically in Legs 137/140 dolerites: (1) green Na- and Ti-rich diopside-hedenbergite partly replaces igneous augite crosscut by an actinolite vein in two samples. By analogy with other occurrences of similar minerals in the pillow section, lithologic transition zone, and upper dike section of Hole 504B, we propose to interpret this secondary clinopyroxene, as well as melanite and fassaite from the pillow section, as resulting from an alteration by late magmatic fluids (deuteric alteration), possibly mixed with small volumes of hydrothermal fluids, at temperatures of less than $500^{\circ}$ to $600^{\circ} \mathrm{C}$; (2) scarce colorless diopside, without anomalous $\mathrm{Ti}$ and $\mathrm{Na}$ concentrations, occurring in veinlets and patches, often associated with amphibole, is interpreted as having crystallized during hydrothermal alteration at minimum temperatures of $500^{\circ}-700^{\circ} \mathrm{C}$.

3. Amphibole is the most widespread and volumetrically abundant secondary mineral in the studied section. Many samples contain a continuous range of amphibole compositions, and there is a relation between the textural occurrence and the type of amphibole: actinolite prevails in veins, whereas actinolitic-hornblende and magnesio-hornblende prevail in the adjacent alteration halos. The lower sheeted dikes drilled during Legs 137/140 experienced higher temperature alteration in general than the shallower dikes, as evidenced by more prevalent actinolitic hornblende and, especially, abundant magnesio- 
Table 7 (continued).

\begin{tabular}{cccccccccccc}
\hline $\mathrm{Si}$ & $\mathrm{Al}$ & $\mathrm{Fe}_{3}$ & $\mathrm{Mn}$ & $\mathrm{Mg}$ & $\mathrm{Ca}$ & $\mathrm{Na}$ & $\mathrm{K}$ & $\mathrm{Ti}$ & $\mathrm{Cr}$ & Total & $\mathrm{XFe}$ \\
\hline 3.018 & 2.232 & 0.666 & 0.000 & 0.012 & 1.999 & 0.005 & 0.000 & 0.004 & 0.064 & 7.999 & 22.968 \\
3.048 & 2.167 & 0.759 & 0.012 & 0.024 & 1.976 & 0.003 & 0.000 & 0.000 & 0.000 & 7.990 & 25.949 \\
3.039 & 2.118 & 0.810 & 0.006 & 0.024 & 1.993 & 0.000 & 0.001 & 0.000 & 0.004 & 7.995 & 27.662 \\
3.071 & 2.071 & 0.769 & 0.005 & 0.106 & 1.877 & 0.005 & 0.000 & 0.006 & 0.064 & 7.974 & 27.093 \\
2.989 & 2.230 & 0.727 & 0.002 & 0.020 & 1.960 & 0.002 & 0.002 & 0.004 & 0.064 & 7.999 & 24.574 \\
3.124 & 2.026 & 0.724 & 0.000 & 0.062 & 1.955 & 0.014 & 0.009 & 0.002 & 0.062 & 7.979 & 26.334 \\
2.998 & 2.106 & 0.831 & 0.003 & 0.008 & 1.966 & 0.009 & 0.001 & 0.011 & 0.062 & 7.996 & 28.298 \\
2.965 & 2.267 & 0.694 & 0.009 & 0.011 & 2.007 & 0.000 & 0.000 & 0.004 & 0.062 & 8.019 & 23.450 \\
2.965 & 2.254 & 0.713 & 0.003 & 0.005 & 1.998 & 0.000 & 0.000 & 0.010 & 0.063 & 8.010 & 24.046 \\
3.001 & 2.428 & 0.513 & 0.011 & 0.002 & 1.972 & 0.005 & 0.001 & 0.003 & 0.062 & 7.997 & 17.437 \\
3.026 & 2.003 & 0.864 & 0.007 & 0.000 & 2.017 & 0.000 & 0.002 & 0.013 & 0.063 & 7.996 & 30.130 \\
3.023 & 2.237 & 0.635 & 0.007 & 0.005 & 2.030 & 0.000 & 0.000 & 0.004 & 0.063 & 8.005 & 22.118 \\
2.993 & 2.227 & 0.708 & 0.011 & 0.034 & 1.967 & 0.000 & 0.000 & 0.000 & 0.066 & 8.006 & 24.129 \\
3.022 & 2.338 & 0.592 & 0.020 & 0.001 & 1.939 & 0.000 & 0.001 & 0.002 & 0.065 & 7.979 & 20.203 \\
2.986 & 2.352 & 0.613 & 0.010 & 0.002 & 1.958 & 0.003 & 0.000 & 0.008 & 0.062 & 7.994 & 20.683 \\
2.939 & 2.421 & 0.600 & 0.011 & 0.002 & 1.969 & 0.000 & 0.000 & 0.008 & 0.062 & 8.012 & 19.872 \\
3.127 & 1.963 & 0.736 & 0.003 & 0.228 & 1.860 & 0.005 & 0.000 & 0.005 & 0.062 & 7.989 & 27.274 \\
3.028 & 2.184 & 0.706 & 0.010 & 0.034 & 1.958 & 0.000 & 0.002 & 0.006 & 0.062 & 7.991 & 24.431 \\
\hline
\end{tabular}

Table 8 (continued).

\begin{tabular}{cccccccccccc}
\hline $\mathrm{Si}$ & $\mathrm{Al}$ & $\mathrm{Fe}_{3}$ & $\mathrm{Mn}$ & $\mathrm{Mg}$ & $\mathrm{Ca}$ & $\mathrm{Na}$ & $\mathrm{K}$ & $\mathrm{Ti}$ & $\mathrm{Cr}$ & $\mathrm{Total}$ & $\mathrm{XFe}_{3}$ \\
\hline 3.060 & 1.758 & 0.172 & 0.000 & 0.064 & 1.900 & 0.007 & 0.023 & 0.000 & 0.003 & 6.988 & 0.090 \\
3.055 & 1.759 & 0.165 & 0.004 & 0.026 & 1.966 & 0.006 & 0.000 & 0.003 & 0.000 & 6.983 & 0.090 \\
2.999 & 1.958 & 0.002 & 0.002 & 0.000 & 1.978 & 0.003 & 0.000 & 0.000 & 0.054 & 6.995 & 0.000 \\
3.054 & 1.950 & 0.003 & 0.000 & 0.000 & 1.873 & 0.011 & 0.000 & 0.000 & 0.055 & 6.947 & 0.000 \\
2.987 & 1.936 & 0.104 & 0.002 & 0.118 & 1.760 & 0.000 & 0.000 & 0.001 & 0.055 & 6.964 & 0.050 \\
2.932 & 1.923 & 0.139 & 0.002 & 0.118 & 1.839 & 0.000 & 0.000 & 0.000 & 0.055 & 7.009 & 0.070 \\
2.980 & 1.941 & 0.103 & 0.014 & 0.080 & 1.876 & 0.000 & 0.000 & 0.000 & 0.002 & 6.997 & 0.050 \\
2.955 & 1.896 & 0.166 & 0.008 & 0.141 & 1.841 & 0.004 & 0.002 & 0.002 & 0.001 & 7.015 & 0.080 \\
2.975 & 1.887 & 0.135 & 0.004 & 0.187 & 1.822 & 0.006 & 0.000 & 0.000 & 0.000 & 7.016 & 0.070 \\
\hline
\end{tabular}

Table 9 (continued).

\begin{tabular}{ccccccccccc}
\hline $\mathrm{Si}$ & $\mathrm{Al}$ & $\mathrm{Fe}_{3}$ & $\mathrm{Mn}$ & $\mathrm{Mg}$ & $\mathrm{Ca}$ & $\mathrm{Na}$ & $\mathrm{K}$ & $\mathrm{Ti}$ & $\mathrm{Cr}$ & Total \\
\hline 26.963 & 9.225 & 0.064 & 0.000 & 0.013 & 4.075 & 0.044 & 0.034 & 0.007 & 0.000 & 40.425 \\
26.721 & 9.398 & 0.084 & 0.000 & 0.020 & 4.272 & 0.053 & 0.012 & 0.000 & 0.007 & 40.567 \\
27.123 & 8.478 & 0.026 & 0.023 & 0.299 & 4.526 & 0.242 & 0.045 & 0.003 & 0.000 & 40.765 \\
27.130 & 8.632 & 0.078 & 0.023 & 0.106 & 4.373 & 0.190 & 0.040 & 0.000 & 0.039 & 40.611 \\
26.676 & 8.580 & 0.380 & 0.012 & 0.523 & 4.540 & 0.165 & 0.018 & 0.007 & 0.019 & 40.920 \\
26.685 & 9.363 & 0.000 & 0.000 & 0.007 & 4.457 & 0.202 & 0.042 & 0.000 & 0.000 & 40.756 \\
26.848 & 9.216 & 0.000 & 0.004 & 0.014 & 4.342 & 0.136 & 0.060 & 0.000 & 0.015 & 40.635 \\
16.530 & 7.560 & 0.074 & 0.013 & 0.009 & 3.424 & 0.058 & 0.023 & 0.002 & 0.000 & 27.691 \\
16.621 & 7.388 & 0.045 & 0.005 & 0.000 & 3.542 & 0.069 & 0.038 & 0.004 & 0.000 & 27.712 \\
16.825 & 7.264 & 0.000 & 0.013 & 0.000 & 3.395 & 0.030 & 0.039 & 0.000 & 0.007 & 27.574 \\
16.208 & 7.757 & 0.000 & 0.000 & 0.000 & 3.930 & 0.036 & 0.000 & 0.000 & 0.000 & 27.931 \\
16.116 & 7.824 & 0.005 & 0.018 & 0.000 & 3.954 & 0.030 & 0.000 & 0.000 & 0.024 & 27.972 \\
\hline
\end{tabular}

hornblendes. The lack of Cl-rich amphiboles may indicate that unusually briney hydrothermal fluids did not develop in the lower sheeted dikes during water-rock interaction.

4. One major new discovery in Hole 504B is the presence of, in some extensively altered vein halos, hydrothermal plagioclase $\left(\mathrm{An}_{80-95}\right)$ that is more calcic than the associated igneous plagioclase $\left(\mathrm{An}_{65-89}\right)$. This secondary plagioclase has been recognized here for the first time in the oceanic crust. The appearance of calcic plagioclase downhole correlates roughly with increases in, but not the first appearance of, magnesio-hornblende. We interpret this hydrothermal anorthite-bytownite, in association with magnesio-hornblende, as being formed in a reaction zone similar to that envisioned for the source of black smoker fluids, from high-temperature $\left(>400^{\circ}-500^{\circ} \mathrm{C}\right), \mathrm{Ca}-$ enriched fluids.

5. Mixed-layer chlorite-smectite exhibit several types of variation in the percentage of chlorite layers present. Chlorite-smectite in veins contains a greater proportion of chlorite layers than that replacing olivine in the host-rock of the same sample, and chlorite-smectite in the rims of olivine pseudomorphs contains a greater percentage of chlorite layers than that at the cores of the pseudomorphs. In the intervals where relict olivine is present and where talc replaces olivine, chlorite-smectites contain relatively lower proportions of chlorite layers (1700-1820, 1905-1938, and 1980-1985 mbsf). Such variations can be explained by differences in temperature of reaction (ranging from $60^{\circ}$ to $320^{\circ} \mathrm{C}$, calculated using Cathelineau geothermometer), or by variations in water-rock ratio and extent of reaction of the rocks.

6. The distribution of $\mathrm{Al}^{\mathrm{IV}}$ and $\mathrm{TiO}_{2}$ of amphiboles with depth is very irregular and reaches maxima at 1630 and $1820 \mathrm{mbsf}$, implying higher temperatures of crystallization. The geothermometry data obtained from chlorite-smectite indicate that three depth levels (1630 1650,1835 , and $1980 \mathrm{mbsf}$ ) probably experienced higher temperature of alteration than the other depth levels. At 1980 mbsf, $\mathrm{Al}^{\mathrm{IV}}$ in amphiboles also reaches a maximum. We know that the highest $\mathrm{Al}^{\mathrm{IV}}$ and Ti contents are mostly represented by amphibole, which crystallizes in the halos and in the host rock, and are interpreted as being prior to chlorite-smectite. We propose that in these three depth zones, the temperature was higher than elsewhere in the Legs $137 / 140 \mathrm{sec}-$ tion during the two first stages of alteration.

7. Spatial relationships and geothermometric arguments allow us to propose the following sequence of alteration:

Stage la, high temperature (less than $500^{\circ}$ to $600^{\circ} \mathrm{C}$ ). It only has a replacement effect, with $\mathrm{Na}$, Ti-rich diopside very locally replacing primary augite along veins. This should be a deuteric or mixed deuteric-hydrothermal stage.

Stage $1 \mathrm{~b}$, high temperature $\left(500^{\circ}-700^{\circ} \mathrm{C}\right)$. Very small amounts of secondary diopside, without anomalous $\mathrm{Na}$ or $\mathrm{Ti}$, crystallize locally in veinlets and patches, possibly prior to Stage 1c, suggesting a water/rock ratio low and then higher.

Stage $1 \mathrm{c}$, high temperature $\left(400^{\circ}-500^{\circ} \mathrm{C}\right)$. Fills open cracks and mainly creates alteration halos around veins and patches. It mainly has a replacement effect, with anorthite replacing plagioclase rim in alteration halos, and hornblende replacing augite, extensively in al- 
teration halos and along the vein edges in the multiply opened veins, and less extensively out of the halos. The low permeability zones did not react to these relatively small amounts of hot fluids.

Stage 2 , lower temperature $\left(250^{\circ}-320^{\circ} \mathrm{C}\right)$. Actinolite and minor chlorite form in the central part of the veins, albite partly replaces primary and secondary An-rich plagioclase, chlorite and chloritesmectite and/or talc (in low permeability zones) replace olivine in the host-rock, and various and abundant amphiboles replace igneous clinopyroxene. The temperature possibly decreased during this stage, leading to the formation of more smectitic clay-minerals.

Stage 3 , relatively high temperature $\left(310^{\circ}-320^{\circ} \mathrm{C}\right)$. Epidote and quartz form in veins and vugs, probably from more evolved fluids. This stage of alteration affects only very slightly the host rock, epidote partially replacing plagioclase in rare instances.

Stage 4, low temperature. Anhydrite crystallizes, probably directly from heated seawater.

Stage 5, low temperature $\left(250^{\circ} \mathrm{C}\right)$. Laumontite and prehnite form.

8. Several lines of evidence suggest that most of the hydrothermal fluids that altered the sheeted dikes at Hole 504B were seawater derived: (1) fluid inclusions in secondary plagioclase have seawaterlike salinities; (2) isotopic signature of altered dolerites suggests seawater contamination; and (3) the high volume of hydrous secondary minerals.

9. Most of the sheeted dike alteration resulted from non-focused, pervasive fluid-rock interaction as opposed to focused, hightemperature, high-W/R discharge alteration. The lower section of dikes of Hole 504B probably represents lower crustal regions that have never been zones of concentrated upwelling of hydrothermal fluids or black smoker vents.

\section{ACKNOWLEDGMENTS}

We wish to thank all the Leg 140 and Leg 148 participants for sharing long sessions of core description and discussions about Hole 504B. We also are grateful to the drilling team for their hard but successful fishing work during Leg 140. Peter Meyer and Peter Schiffman are thanked for their careful and constructive review of this manuscript. This work was supported by CNRS-INSU (92/ATP/734) to C.L. and by the National Science Foundation and JOI/USSSP to D.V. C.L. expresses eternal thanks to Y. Lancelot and P. Pezard for their help during the 10 hours preceding the beginning of Leg 140, and to $\mathrm{H}$. Bohn.

\section{REFERENCES}

Agrinier, P., and Agrinier, B., in press. Comment on the knowledge of the depth of a rock sample from a drilled core. Sci. Drilling.

Alt, J.C., 1984. The structure, chemistry, and evolution of a submarine hydrothermal system, DSDP Site 504 [Ph.D. dissert.]. Univ. of Miami, Miami, FL.

Alt, J.C., Anderson, T.F., and Bonnell, L., 1989a. The geochemistry of sulfur in a $1.3 \mathrm{~km}$ section of hydrothermally altered oceanic crust, DSDP Hole 504B. Geochim. Cosmochim. Acta, 53:1011-1023.

Alt, J.C., Anderson, T.F., Bonnell, L., and Muehlenbachs, K., 1989b. Mineralogy, chemistry, and stable isotopic compositions of hydrothermally altered sheeted dikes: ODP Hole 504B, Leg 111. In Becker, K., Sakai, H., et al., Proc. ODP, Sci. Results, 111: College Station, TX (Ocean Drilling Program), 27-40.

Alt, J.C., Honnorez, J., Laverne, C., and Emmermann, R., 1986a. Hydrothermal alteration of a $1 \mathrm{~km}$ section through the upper oceanic crust, Deep Sea Drilling Project Hole 504B: the mineralogy, chemistry and evolution of seawater-basalt interactions. J. Geophys. Res., 91:10309-10335.

\footnotetext{
Abbreviations for names of organizations and publications in ODP reference lists follow the style given in Chemical Abstracts Service Source Index (published by American Chemical Society).
}

Alt, J.C., Laverne, C., and Muehlenbachs, K., 1985. Alteration of the upper oceanic crust: mineralogy and processes in Deep Sea Drilling Project Hole 504B, Leg 83. In Anderson, R.N., Honnorez, J., Becker, K., et al., Init. Repts. DSDP, 83: Washington (U.S. Govt. Printing Office), 217-247.

Alt, J.C., Muehlenbachs, K., and Honnorez, J., 1986b. An oxygen isotopic profile through the upper kilometer of the oceanic crust, DSDP Hole 504B. Earth Planet. Sci. Lett., 80:217-229.

Anderson, R.N., Honnorez, J., Becker, K., Adamson, A.C., Alt, J.C., Emmermann, R., Kempton, P.D., Kinoshita, H., Laverne, C., Mottl, M.J., and Newmark, R.L., 1982. DSDP Hole 504B, the first reference section over $1 \mathrm{~km}$ through Layer 2 of the oceanic crust. Nature, 300:589-594.

Anderson, R.N., Honnorez, J., Becker, K., et al., 1985. Init. Repts. DSDP, 83: Washington (U.S. Govt. Printing Office).

Becker, K., Sakai, H., et al., 1988. Proc. ODP, Init. Repts., 111: College Station, TX (Ocean Drilling Program).

, 1989. Proc. ODP, Sci. Results, 111: College Station, TX (Ocean Drilling Program).

Berndt, M.E., and Seyfried, W.E., Jr., 1993. Ca and $\mathrm{Na}$ exchange during hydrothermal alteration of calcic plagioclase at $400^{\circ} \mathrm{C}$ and 400 bars. Geochim Cosmochim Acta, 57:4445-4451.

Bettison, L.A., and Schiffman, P., 1988. Compositional and structural variations of phyllosilicates from the Point Sal ophiolite, California. Am. Mineral., 73:62-76.

Bevins, R.E., Robinson, D., and Rowbotham, G., 1991. Compositional variations in mafic phyllosilicates from regional low-grade metabasites and application of the chlorite geothermometer. J. Metamorph. Geol., 9:711721.

Blundy, J.D., and Holland, T.J.B., 1990. Calcic amphibole equilibria and a new amphibole-plagioclase geothermometer. Contrib. Mineral. Petrol., 104:208-224.

Cathelineau, M., 1988. Cation site occupancy in chlorites and illites as a function of temperature. Clay Mineral., 23:471-485.

Cathelineau, M., and Nieva, D., 1985. A chlorite solid solution geothermometer: the Los Azufres (Mexico) geothermal system. Contrib. Mineral. Petrol., 91:235-244.

Harper, G.D., Bowman, J.R., and Kuhns, R., 1988. A field, chemical and stable isotopic study of subseafloor metamorphism of the Josephine Ophiolite, California-Oregon. J. Geophys. Res., 93:4625-4656.

Hey, M.H., 1954. A new review of the chlorites. Mineral. Mag., 30:277.

Honnorez, J., Alt, J.C., Honnorez-Guerstein, B.-M., Laverne, C., Muehlenbachs, K., Ruiz, J., and Saltzman, E., 1985. Stockwork-like sulfide mineralization in young oceanic crust: Deep Sea Drilling Project Hole 504B. In Anderson, R.N., Honnorez, J., Becker, K., et al., Init. Repts. DSDP, 83: Washington (U.S. Govt. Printing Office), 263-282.

Honnorez, J., Laverne, C., Hubberten, H.-W., Emmermann, R., and Muehlenbachs, K., 1983. Alteration processes in Layer 2 basalts from Deep Sea Drilling Project Hole 504B, Costa Rica Rift. In Cann, J.R., Langseth, M.G., Honnorez, J., Von Herzen, R.P., White, S.M., et al., Init. Repts. DSDP, 69: Washington (U.S. Govt. Printing Office), 509-546.

Ishizuka, H., 1989. Mineral paragenesis of altered basalts from Hole 504B, ODPLeg 111. In Becker, K., Sakai, H., et al., Proc. ODP, Sci. Results, 111: College Station, TX (Ocean Drilling Program), 61-76.

Laird, J., and Albee, A.L., 1981. Pressure, temperature, and time indicators in mafic schist: their application to reconstructing the polymetamorphic history of Vermont. Am. J. Sci., 281:97-126.

Laverne, C., 1983. Occurrence of melanite and aegirine-augite in Deep Sea Drilling Project Hole 504B. In Cann, J.R., Langseth, M.G., Honnorez, J., Von Herzen, R.P., White, S.M., et al., Init. Repts. DSDP, 69: Washington (U.S. Govt. Printing Office), 593-605.

, 1987a. Unusual occurrence of aegirine-augite, fassaite and melanite in oceanic basalts (DSDP Hole 504B). Lithos, 20:135-151.

, 1987b. Les altérations des basaltes en domaine océanique: minéralogie, pétrologie et géochimie d'un système hydrothermal: le puits 504B, Pacifique oriental [Thèse]. Univ. Aix-Marseille III.

Laverne, C., Honnorez, J., and Alt, J.C., 1989. Transition entre l'altération à basse température et le métamorphisme hydrothermal de la croûte océanique: étude pétrographique et géochimique du puits 504B, Est-Pacifique. Bull. Soc. Geol. Fr., 8:327-337.

Leake, B.E., 1978. Nomenclature of amphiboles. Am. Mineral., 63:10231053.

Lindsley, D.H., 1983. Pyroxene thermometry. Am. Mineral., 68:477-493.

Lindsley, D.H., and Anderson, D.J., 1983. A two-pyroxene thermometer. $J$. Geophys. Res., 88 (Suppl.):A887-A906. 
Liou, J.G., and Ernst, W.G., 1979. Oceanic ridge metamorphism of the East Taiwan Ophiolite. Contrib. Mineral. Petrol., 68:335-348.

Liou, J.G., Kuniyoshi, S., and Ito, K., 1974. Experimental studies of the phase relations between greenschist and amphibolite in a basaltic system. Am. .J. Sci., 274:613-632.

Mora, C.I., and Ramseyer, K., 1992. Cathodoluminescence of coexisting plagioclases, Boehls Butte anorthosite: CL activators and fluid flow paths. Am. Mineral., 77:1258-1265.

Morimoto, N., 1988. Nomenclature of pyroxenes. Bull. Minéral., 111:535550.

Nyström, J.O., 1983. Pumpellyite-bearing rocks in Central Sweden and extent of host rock alteration as a control of pumpellyite composition. Contrib. Mineral. Petrol.. 83:159-168.

Raase, P., 1974. Al and Ti contents of hornblende: indicators of pressure and temperature of regional metamorphism. Contrib. Mineral. Petrol., $45: 231-236$

Schiffman, P., and Fridleifsson, G.O., 1991. The smectite-chlorite transition in drillhole $\mathrm{Nj}-15$, Nesjavellir geothermal field, Iceland: XRD, BSE and electron microprobe investigation. J. Metamorph. Geol., 9:679-696.

Shau, Y.H., and Peacor, D.R., 1992. Phyllosilicates in hydrothermally altered basalts from DSDP Hole 504B, Leg 83. A TEM and AEM study. Contrib. Mineral. Petrol., 112:119-133.

Shipboard Scientific Party, 1988. Site 504: Costa Rica Rift. In Becker, K. Sakai, H., et al., Proc. ODP, Init. Repts., 111: College Station, TX (Ocean Drilling Program), 35-251.

, 1992a. Site 504. In Becker, K., Foss, G., et al., Proc. ODP, Init. Repts., 137: College Station, TX (Ocean Drilling Program), 15-55.

, 1992b. Site 504. In Dick, H.J.B., Erzinger, J., Stokking, L.B., et al. Proc. ODP, Init. Repts., 140: College Station, TX (Ocean Drilling Program), 37-200.
Spear, F.S., 1980. NaSi-CaAl exchange equilibrium between plagioclase and amphibole, an empirical model. Contrib. Mineral. Petrol., 72:33-41.

Stakes, D., Mével, C., Cannat, M., and Chaput, T., 1991. Metamorphic stratigraphy of Hole 735B. In Von Herzen, R.P., Robinson, P.T., et al., Proc. ODP, Sci. Results, 118: College Station, TX (Ocean Drilling Program), 153-180.

Stakes, D.S., and O'Neill, J.R., 1982. Mineralogy and stable isotope geochemistry of hydrothermally altered oceanic rocks. Earth Planet. Sci. Lett., 57:285-304.

Vanko, D.A., 1986. High-chlorine amphiboles from oceanic rocks: product of highly saline hydrothermal fluids? Am. Mineral., 71:51-59.

Vanko, D.A., Griffith, J.D., and Erickson, C.L., 1992. Calcium-rich brines and other hydrothermal fluids in fluid inclusions from plutonic rocks. Oceanographer Transform, Mid-Atlantic Ridge. Geochim. Cosmochim. Acta, 56:35-47.

Vanko, D.A., and Stakes, D.S., 1991. Fluids in oceanic layer 3: evidence from veined rocks, Hole 735B, Southwest Indian Ridge. In Von Herzen, R.P., Robinson, P.T., et al., Proc. ODP, Sci. Results, 118: College Station, TX (Ocean Drilling Program), 181-218.

Von Damm, K.L., Edmond, J.M., Grant, B., Measures, C.I., Walden, B., and Weiss, R.F., 1985. Chemistry of submarine hydrothermal solutions at $21^{\circ} \mathrm{N}$, East Pacific Rise. Geochim. Cosmochim. Acta, 49:2197-2220.

Date of initial receipt: 24 May 1993

Date of acceptance: 14 April 1994

Ms 137/140SR-014

Table 10. Representative titanite analyses, Hole 504B, Legs 137/140.

\begin{tabular}{ccccccccccccccc}
\hline $\begin{array}{c}\text { Core, section, } \\
\text { interval (cm) }\end{array}$ & Analysis & $\mathrm{SiO}_{2}$ & $\mathrm{Al}_{2} \mathrm{O}_{3}$ & $\mathrm{Fe}_{2} \mathrm{O}_{3 \mathrm{t}}$ & $\mathrm{MnO}$ & $\mathrm{MgO}$ & $\mathrm{CaO}$ & $\mathrm{Na}_{2} \mathrm{O}$ & $\mathrm{K}_{2} \mathrm{O}$ & $\mathrm{TiO}_{2}$ & $\mathrm{Cr}_{2} \mathrm{O}_{3}$ & $\mathrm{Cl}$ & $\mathrm{ZnO}^{2}$ & $\mathrm{Total}$ \\
\hline $173 \mathrm{R}-1,77-79$ & $\mathrm{~B} 2179$ & 30.18 & 1.42 & 0.75 & 0.00 & 0.00 & 28.58 & 0.17 & 0.00 & 37.95 & 0.00 & 0.03 & 0.00 & 99.05 \\
& $\mathrm{~B} 2180$ & 30.36 & 1.51 & 0.84 & 0.02 & 0.01 & 29.05 & 0.15 & 0.19 & 37.31 & 0.00 & 0.12 & 0.00 & 99.44 \\
$177 \mathrm{R}-1,11-15$ & $\mathrm{~B} 2116$ & 33.67 & 1.90 & 3.83 & 0.02 & 1.97 & 26.98 & 0.07 & 0.01 & 32.14 & 0.02 & 0.03 & 0.00 & 100.61 \\
& $\mathrm{~B} 2117$ & 31.37 & 2.69 & 1.39 & 0.10 & 0.00 & 28.60 & 0.02 & 0.02 & 35.49 & 0.15 & 0.02 & 0.00 & 99.83 \\
& $\mathrm{~B} 2121$ & 30.72 & 1.54 & 1.04 & 0.03 & 0.04 & 29.64 & 0.02 & 0.00 & 37.39 & 0.00 & 0.00 & 0.13 & 100.42 \\
$237 \mathrm{R}-1,24-26$ & $\mathrm{~B} 3007$ & 31.30 & 3.87 & 1.93 & 0.01 & 0.00 & 28.81 & 0.00 & 0.00 & 32.84 & 0.06 & 0.03 & 0.00 & 98.82 \\
\hline
\end{tabular}

Note: Structural formulas are calculated for 20 oxygens.

Table 10 (continued).

\begin{tabular}{cccccccccccr}
\hline $\mathrm{Si}$ & $\mathrm{Ti}$ & $\mathrm{Al}$ & $\mathrm{Fe}^{3+}$ & $\mathrm{Cr}$ & $\mathrm{Mn}$ & $\mathrm{Zn}$ & $\mathrm{Mg}$ & $\mathrm{Ca}$ & $\mathrm{Na}$ & $\mathrm{K}$ & Total \\
\hline 3.984 & 3.767 & 0.221 & 0.068 & 0.000 & 0.000 & 0.000 & 0.000 & 4.042 & 0.044 & 0.000 & 12.126 \\
4.000 & 3.697 & 0.234 & 0.075 & 0.000 & 0.002 & 0.000 & 0.002 & 4.101 & 0.038 & 0.032 & 12.183 \\
4.348 & 3.121 & 0.289 & 0.335 & 0.002 & 0.002 & 0.000 & 0.379 & 3.733 & 0.018 & 0.002 & 12.228 \\
4.095 & 3.484 & 0.414 & 0.123 & 0.015 & 0.011 & 0.000 & 0.000 & 4.000 & 0.005 & 0.003 & 12.150 \\
4.007 & 3.668 & 0.237 & 0.092 & 0.000 & 0.003 & 0.013 & 0.008 & 4.143 & 0.005 & 0.000 & 12.163 \\
4.125 & 3.255 & 0.601 & 0.173 & 0.006 & 0.001 & 0.000 & 0.000 & 4.068 & 0.000 & 0.000 & 12.230 \\
\hline
\end{tabular}



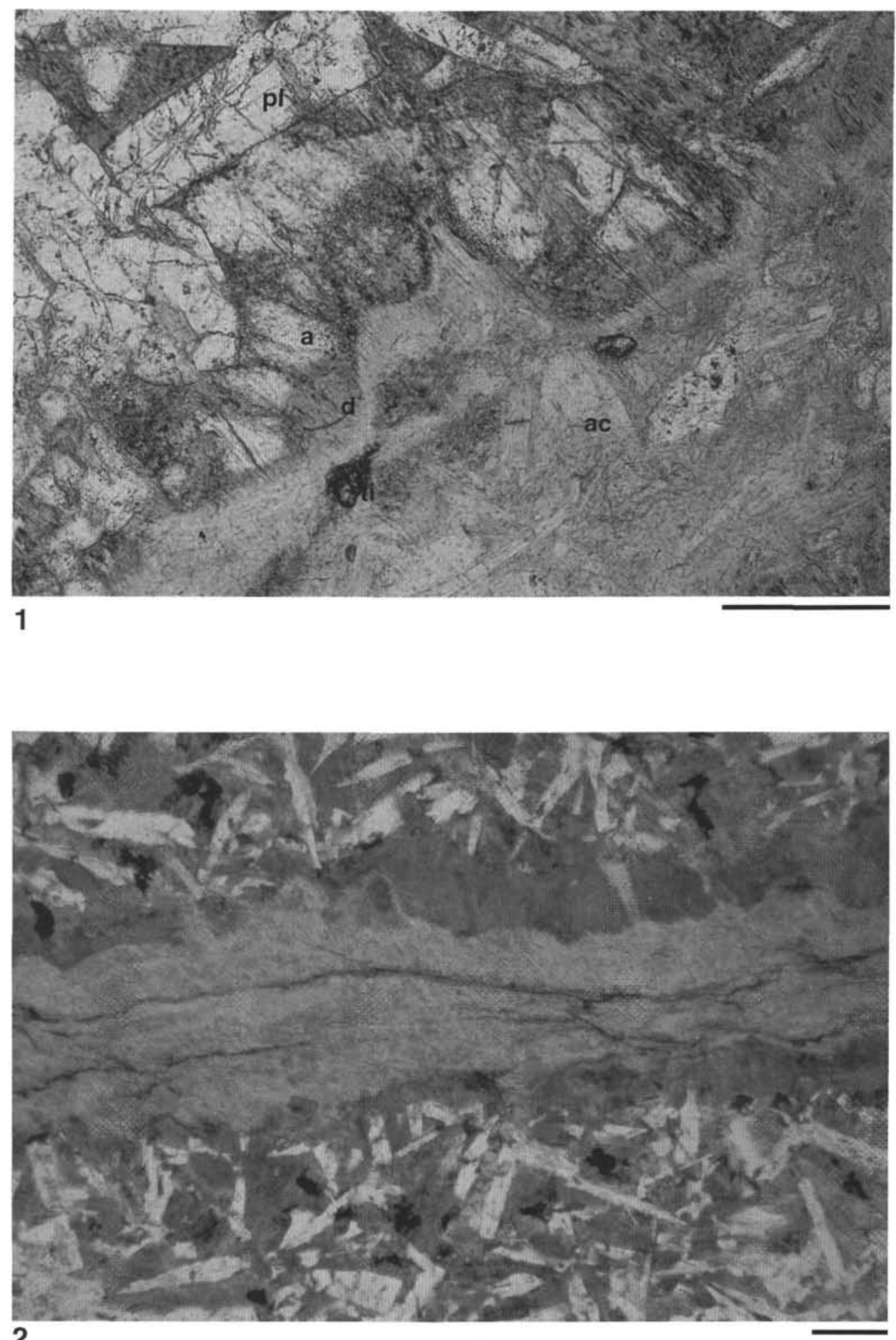

2

Plate 1. 1. Secondary Na-, Ti-diopside (d) replacing igneous augite (a) in contact with an actinolite (ac) + titanite (ti) vein in Sample 137-504B-180M-1, 74-78 cm (Piece 7) (plane polarized light). pl = plagioclase. Scale bar is $0.2 \mathrm{~mm}$. 2. Actinolite vein, hornblende in adjacent alteration halo in Sample 140-504B-197R-1, 100-102 cm (Piece 22) (plane polarized light). Scale bar is $0.5 \mathrm{~mm}$. 

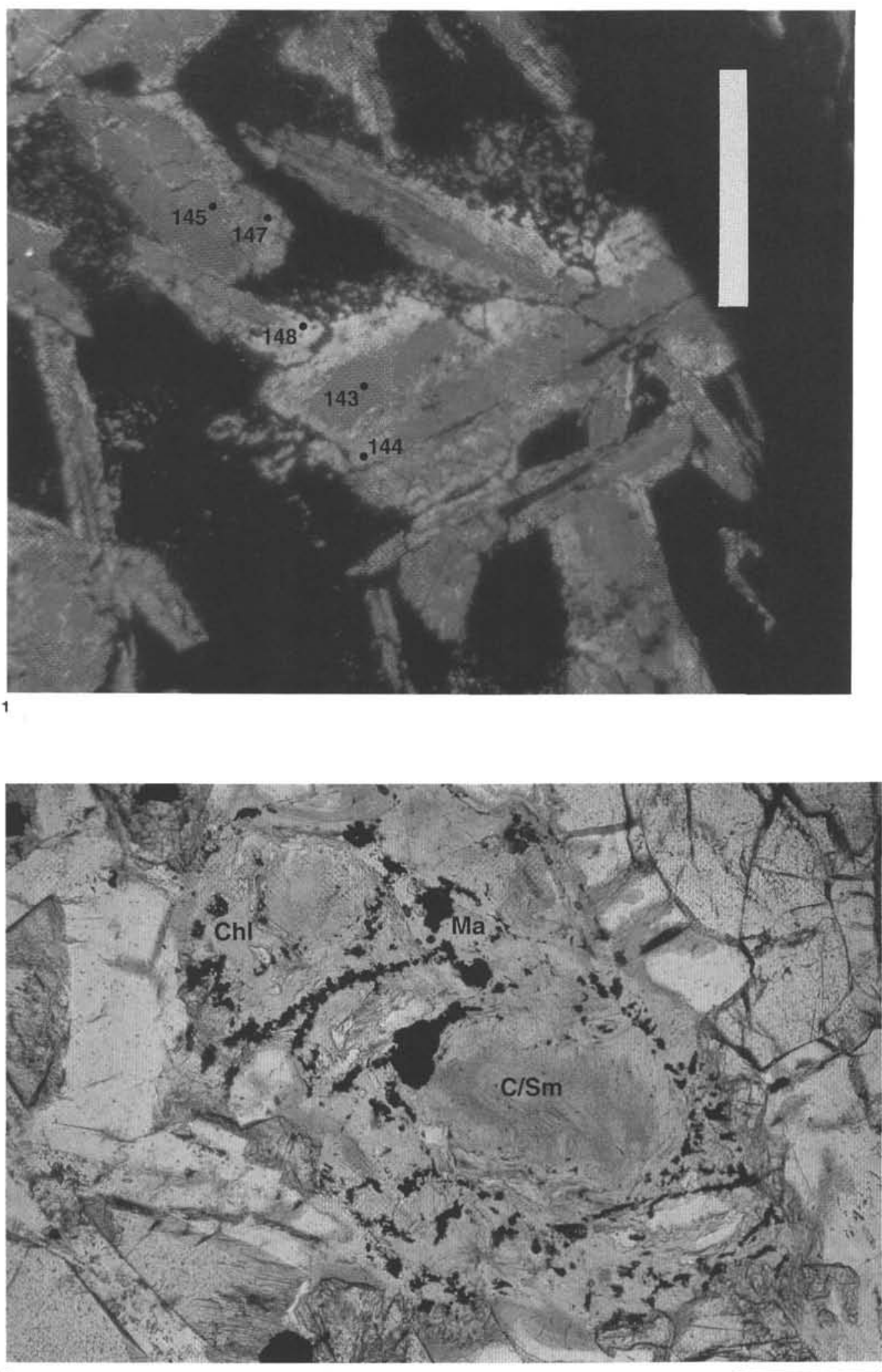

2

Plate 2. 1. Photomicrograph of dolerite from a vein halo, Sample 140-504B-205R-1, 13-16 cm (Piece 1), taken with a cathodoluminescence microscope (17 $\mathrm{KeV}$ accelerating voltage, $0.5 \mathrm{~mA}$ beam current). The field of view is from a well-developed alteration halo along an amphibole vein. Igneous plagioclase laths luminesce blue-gray and possess thick incomplete rims of greenish hydrothermal calcic plagioclase. Interstitial amphibole is nonluminescent. Electron microprobe analysis spots are labeled and are given in Table 4. Plagioclase cores are $\mathrm{An}_{74-77}$, whereas the rims are $\mathrm{An}_{89-94}$. Late-stage crosscutting veinlets of nonluminescent albite are $\mathrm{An}_{11}$. Scale bar is $0.5 \mathrm{~mm}$. 2. Olivine phenocryst replaced by chlorite (Chl) + magnetite (Ma) at the rim (analyses B3104, B5009, B5014; Table 5), mixed-layer chlorite-smectite (C/Sm) in the core (analyses B3103, B5025, B5026; Table 5) in Sample 140-504B-208R-2, 76-81 cm (Piece 13) (plane polarized light). Scale bar is $0.2 \mathrm{~mm}$. 